NBSIR 82-2491

\title{
Test Procedures for the Determination of the Gross Calorific Value of Refuse and Refuse-Derived-Fuel of Kilogram-Size Samples Using Constant Pressure Flow Calorimeter
}

Summary of the 1980 Fiscal Year Results

U.S. DEPARTMENT OF COMMERCE National Bureau of Standards Chemical Thermodynamics Division Center for Chemical Physics and the

Temperature and Pressure Measurements and Standards Division Center for Absolute Physical Quantities

Washington, DC 20234

June 1982

Prepared for

U.S. Department of Energy

Office of Energy from Municipal Waste

Forrestal Building

- 0 Independence Avenue, SW

QC shington, DC 20585

100

.456

$\forall 82-2491$ 



\section{TEST PROCEDURES FOR THE}

DETERMINATION OF THE GROSS

CALORIFIC VALUE OF REFUSE AND

REFUSE-DERIVED-FUEL OF

KILOGRAM-SIZE SAMPLES USING

CONSTANT PRESSURE FLOW

\section{CALORIMETER}

Summary of the 1980 Fiscal Year Results

D.R. Kirklin, J.C. Colbert, K.L. Churney, M.L. Reilly*,

D.D. Thornton*, R.V. Ryan, A.E. Ledford, and E.S. Domalski

U.S. DEPARTMENT OF COMMERCE

National Bureau of Standards

Chemical Thermodynamics Division

Center for Chemical Physics

and the

Temperature and Pressure Measurements and Standards Division

Center for Absolute Physical Quantities

Washington, DC 20234

June 1982

Prepared for

U.S. Department of Energy

Office of Energy from Municipal Waste

Forrestal Building

1000 Independence Avenue, SW

Washington, DC 20585

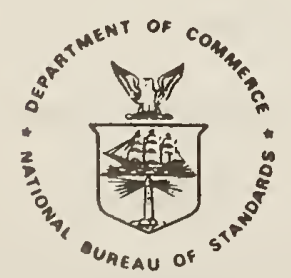

U.S. DEPARTMENT OF COMMERCE, Malcolm Baldrige, Secretary NATIONAL BUREAU OF STANDARDS, Ernest Ambler, Director 



\section{Preface}

This report presents the results of studies carried out during fiscal year 1980 to determine thermal properties of municipal solid waste (MSW) which are needed to adequately utilize MSW as an alternate fuel source. The most significant thermal property of a fuel is its calorific value. Therefore, we have been primarily concerned with the information necessary to make a precise and accurate measurement of the calorific value of MSW.

Calorific values are traditionally determined by oxygen bomb calorimetry of gram-size samples. The precise and accurate bomb calorimetric methods that are presently being used require that substances under study be homogeneous. Such substances present no sampling problem when gram-size amounts are extracted from larger quantities of the same substance. However, representative samples of multi-ton quantities of heterogeneous materials, such as MSW, must be processed to a small particle size (2 mm or less) to produce a "homogeneous" material from which to extract a gram-size sample for bomb calorimetric studies. Doubt often exists as to whether the gram-size samples are truly representative of the multi-ton quantities of MSW. Also, doubt still persists as to whether the true calorific value (i.e., the accurate calorific value) of the entire MSW population is obtainable from gram-size samples. Therefore, a need exists to enhance the state-of-the-art of calorific measurements so that kilogram-size samples of heterogeneous materials can be accommodated satisfactorily.

We have studied the variability of MSW to determine the problems that are associated with the sampling of MSW in gram-size amounts. We have also designed and built an oxygen flow calorimeter in which to measure the calorific value of 25 gram samples of MSW and are presently building a flow 

calorimeter for 2.5 kilogram samples of MSW.

This report is also a partial response to the Resource Conservation and Recovery Act of 1976, PL 94-580, which mandates the National Bureau of Standards (NBS) to provide guidelines for the development of specifications for the classification of materials which would otherwise be destined for disposal. In conjunction with this legislation, NBS has been working with standards setting organizations, such as the Americal Society of Testing and Materials (ASTM) and the American Society of Mechanical Engineers (ASME), to provide the specifications necessary to establish MSW as an alternative fuel and as a recoverable energy source. The ASTM Committee E-38 on Resource Recovery and its Subcommittee E-38.01 on energy are concerned with consensus standards necessary for establishing refuse-derived-fuel (RDF) as an article of commerce. The ASME PTC 33 Committee is concerned with establishing performance test codes (PTC) for boilers and incinerators which are designed to burn MSW. Both standards setting organizations need reliable and credible methods for determining the calorific value of MSW.

This report is divided into three parts and illustrates the systematic approach used at NBS to design, construct, and place into operation a constant pressure flow calorimeter which can accommodate kilogram-size samples .

Part A The Variability of Municipal Solid Waste and its Relationship to the Determination of the Calorific Value of Refuse-Derived-Fuels.

Part B 25 Gram Capacity Combustion Flow Calorimeter.

Part C Trial Combustions of Kilogram-Size Samples of Municipal Solid Waste. 
Part $A$ is concerned with how the variability of MSW effects the measurement of the calorific value using gram-size samples. Part B demonstrates the suitability of oxygen flow calorimetry for measuring the calorific value of MSW using 25 gram samples of RDF. Part $C$ is concerned with the determination of the combustion characteristics of kilogram-size samples of MSW.

Our primary objective is to develop a method for the determination of accurate calorific values for minimally processed MSW and to correlate these calorific values with those obtained on RDF samples which have been processed to a small particle size (i.e., $<2 \mathrm{~mm}(2 \mathrm{~mm}(0.08 \mathrm{in})$ ) for measurement of calorific values on gram-size samples. 
Part A. THE VARIABILITY OF MUNICIPAL SOLID WASTE AND ITS RELATIONSHIP TO THE DETERMINATION OF THE CALORIFIC VALUE OF REFUSE-DERIVED FUELS D.R. Kirklin, J.C. Colbert, P.H. Decker, A.E. Ledford, R.V. Ryan, and E.S. Domalski

Abstract

1. Introduction

2. Experimental

2.1 Collection of MSW Samples

2.2 Processing

2.3 Procedure

3. Discussion of Results

4. Statistical Analysis of Results A-12

5. Conclusions

6. Acknowledgements, A-15

7. References

Part B. 25 GRAM CAPACITY COMBUSTION FLOW CALORIMETER

E.S. Domalski, K.L. Churney, M.L. Reilly, D.R. Kirklin, A.E. Ledford, and D.D. Thornton

1. Introduction

2. 25 Gram Flow Calorimeter Experimental Work B-4

2.1 Apparatus and Samples B-4

2.2 Description of a Typical Experiment B-8

3. Calculation of 25 Gram Flow Results B-10

3.1 Calculation of $\Delta T \quad B-11$

3.2 Calculation of Enthalpy Changes B-14 
3.3 Calculation of $\Delta H_{s t}$ from Bomb Calorimetry

3.4 Calculation of $\Delta \mathrm{H}_{\text {ic }}$

3.5 Calculation of $\Delta \mathrm{H}_{\mathrm{g}}$

3.6 Calculation of $\Delta H_{v}$

3.7 Calculation of $\Delta \mathrm{H}_{\text {ign }}$

3.8 Summary of Calculations

4. Systematic Error Analysis of 25 Gram Flow Experiments

4.1 Errors in $\Delta R$

4.2 Errors in $\Delta \mathrm{H}_{\text {st }}$

4.3 Errors in $\Delta H_{i c}$

4.4 Errors in $\Delta \mathrm{H}_{\mathrm{g}}$

4.5 Errors in $\Delta H_{v}$

4.6 Summary of Error Analysis

5. Results of 25 Gram Flow Experiments and Conclusions

6. References

7. Acknowledgements

Part C. TRIAL COMBUSTIONS OF KILOGRAM-SIZE SAMPLES OF MUNICIPAL SOLID WASTE A.E. Ledford, R.V. Ryan, M.L. Reilly, E.S. Domalski, and K.L. Churney Abstract

1. Introduction

2. Apparatus and Samples

3. Description of a Typical Experiment

4. Summary of Results

5. References 
PART A. THE VARIABILITY OF MUNICIPAL SOLID WASTE AND ITS RELATIONSHIP TO THE DETERMINATION OF THE CALORIFIC VALUE OF REFUSE-DERIVED-FUELS

Table 1 Sample Collection Schedule

Table 2 Test Data

Table 3 Total Ash and Heating Values

Table 4 Total Heating Values

Table 5 Summary of Results

PART B. 25 GRAM CAPACITY COMBUSTION FLOW CALORIMETER

Table 1 Flow Calorimetric Measurements on RDF-4 Samples; Calculation of the Corrected Temperature Rise

Table 2 Bomb Calorimetric Measurements on RDF-4 Samples

Table 3 Standard Flow'Reaction

Table 4 Conversion of Calorimetric Data From Bomb to Flow Conditions

Table 5 Calculation of Moles of Water Vaporized From the Calorimeter

Table 6 Sample Mass and Molar Quantities for Flow Calculations

Table 7 Calculation of 25 Gram Flow Calorimeter Results

Table 8 Acid Content of Water Trap

Table 9 Systematic Error Analysis of 25 Gram Flow Experiments

Table 10 Summary of 25 Gram Flow Results

Table 11 Comparision of Flow and Bomb Calorimeter Results 


\section{List of Figures}

PART A. THE VARIABILITY OF MUNICIPAL SOLID WASTE AND ITS RELATIONSHIP TO THE DETERMINATION OF THE CALORIFIC VALUE OF REFUSE-DERIVED-FUELS

Figure 1 RDF-2 Sample Processing Scheme

Figure 2 Variability of Total Moisture

Figure 3 Variability of Residual Moisture

Figure 4 Variability of Bomb Ash

Figure 5 Variability of Furnace Ash

Figure 6 Variability of Non-Millable Material

Figure 7 Variability of Total Bomb Ash

Figure 8 Variability of Total Furnace Ash

Figure 9 Variability of HHVI

Figure 10 Variability of HHV2

Figure 11 Variability of HHV3-F

Figure 12 Variability of HHV3-B

Figure 13 Effect of Sample Split on Furnace Ash

Figure 14 Effect of Sample Split on Bomb Ash

Figure 15 Effect of Sample Split on HHVI

Figure 16 Correlation of Higher Heating Value and Furnace Ash

Figure 17 Correlation of Higher Heating Value and Bomb Ash

PART B. 25 GRAM CAPACITY COMBUSTION FLOW CALORIMETER

Figure 1 The 25 Gram Combustor

Figure 2 The 25 Gram Flow Calorimeter 
Figure 3 The $\mathrm{CO}-\mathrm{CO}_{2}$ Composition of the Product Gases vs.

Elapsed Time From Ignition for a Typical Experiment

PART C. TRIAL COMBUSTIONS OF KILOGRAM-SIZE SAMPLES OF MUNICIPAL SOLID WASTE

Figure 1 The 2.5 Kilogram Combustor

Figure 2 The Configuration of the Lower Chamber of the Combustor for Experiments 17 and 18 

PART A. THE VARIABILITY OF MUNICIPAL SOLID WASTE AND ITS RELATIONSHIP TO THE DETERMINATION OF THE CALORIFIC VALUE OF REFUSE-DERIVED FUELS

D.R. Kirklin, J.C. Colbert, P.H. Decker, A.E. Ledford, R.V. Ryan, and E.S. Domalski

\section{Abstract}

A study was carried out to examine the variability, over a two-week period, of municipal solid waste (MSW) at the Baltimore County Resource Recovery Facility in Cockeysville, Maryland. Samples of municipal solid waste which had been processed through a primary shredder were collected daily for two weeks. After the total moisture content was determined, the samples were reduced in particle size to $2 \mathrm{~mm}$ or less. A total of 40 samples were prepared for measurements. Testing was carried out for residual moisture, furnace ash, bomb ash, and calorific or higher heating value.

The daily variability (i.e., excluding the within bag variability) of MSW is $36 \%$ and $37 \%$ for moisture and ash, respectively. The combustible fraction of MSW is directly related to the moisture and bomb-ash free higher heating value (HHV3-B) which has a daily variability (i.e., excluding the within bag variability) of only $4 \%$. Statistical analysis of the data suggests that the day to day variability of MSW constitutes 70 to $80 \%$ of the overall variability, with the other variables being errors in sampling, size reduction procedures, and measurement techniques.

\section{Introduction}

The selection of representative samples from large quantities of municipal solid waste (MSW) for standard laboratory tests is difficult because of the heter- 
ogenity of MSW. It is the opinion of many in the field that gram-size test samples are too small to be representative of multi-ton quantities of MSW. Hence, the results of any analysis using gram-size samples can be in doubt.

The influence of processing MSW to 2.0 or $0.5 \mathrm{~mm}$ particle size is also of concern. Such size reduction is needed in order to follow certain ASTM standard methods of test on gram amounts of sample. Some suggest that this size reduction can inadvertently induce segregation of heavy and light particles and possibly even bring about significant changes in chemical composition, although there is no evidence on either side of this question.

The above considerations are important in the decision of how large a sample must be to be representative. However, before such a decision can be reached, information on the variability of MSW must be known as it is encountered in the waste stream (i.e., hour to hour, day to day, week to week, etc.), as well as comparison of the magnitude of this variability to estimated errors (random and systematic).

A study has been carried out to determine the variability of MSW which was examined over a two week period at the Baltimore County Resource Recovery Facility which is operated by the Teledyne National Corporation in Cockeysville, Maryland. At this facility, the MSW samples studied were processed only through the primary shredder. MSW which has been processed through the primary shredder is often referred to as refuse-derived fuel-2 (RDF-2). This material is defined as waste processed to coarse particle size with or without ferrous metal separation [1].

The objective of this study is to determine the short-time (i.e., two week period) variability of as received MSW which has received minimal processing which is characteristic of mass burning facilities. The variabilities will be largely in the moisture and ash contents and therefore will not be extendable to more highly processed RDF which may have a smaller ash content. 
2. Experimental ${ }^{*}$

2.1 Collection of MSW Samples

Table 1 shows the schedule used for collection of the samples. Two samples (or bags) of RDF-2 were collected daily for two weeks. A pair of samples were obtained on mornings and afternoons of successive days. Each individual bag weighed approximately $2.5 \mathrm{~kg}$ and was taken consecutively off a conveyor belt prior to the usual magnetic separation. The conveyor belt was stopped and approximately a one foot section across the entire width of the betl was removed. The sample was collected until the one foot segment of the belt contained no RDF.

TABLE 1. Sample Collection Schedule

Week 1 (Jan 14-18, 1980)

Week 2 (Jan 21-25, 1980)

Day

$\begin{array}{lllll}M & T & \text { Th } & \mathrm{F}\end{array}$

$M \quad T W$ Th $F$

Time of PM AM PM AM PM

Sampling

No. of $2 \quad 2 \quad 2 \quad 2 \quad 2$

$\begin{array}{lllll}2 & 2 & 2 & 2 & 2\end{array}$

bags

Although trade names, actual sources from which samples were obtained, specific apparatus and/or its manufacturer has been identified, the National Bureau of Standards disclaims any endorsement or recommendation of use of the commercial materials or apparatus used in this scientific investigation.

\subsection{Processing}

The bags were delivered daily to the laboratory, weighed, and their contents placed in a drying oven overnight at $105{ }^{\circ} \mathrm{C}$. The dried samples were reweighed the following morning to determine the total moisture content. Bags from each day were labelled, \#1 and \#2. For simplicity, the processing of bag \#1 of a pair is described below and is illustrated in the flow diagram in Fig. 1. Bag \#2 was treated identically. 
Statistical Study of Baltimore County RDF.2

PRIMARY
SHRED

PIECES 10 TO $15 \mathrm{~cm}$ IN DIMENSION.

$2.5 \mathrm{~kg}$ RDF-2 SAMPLE

RDF.2 SAMPLE WAS COLLECTED AT THE TELEDYNE NATIONAL FACILITY.

REDUCED TO $2 \mathrm{~cm}$. REDUCED TO $2 \mathrm{~mm}$. MIXED, SPLIT

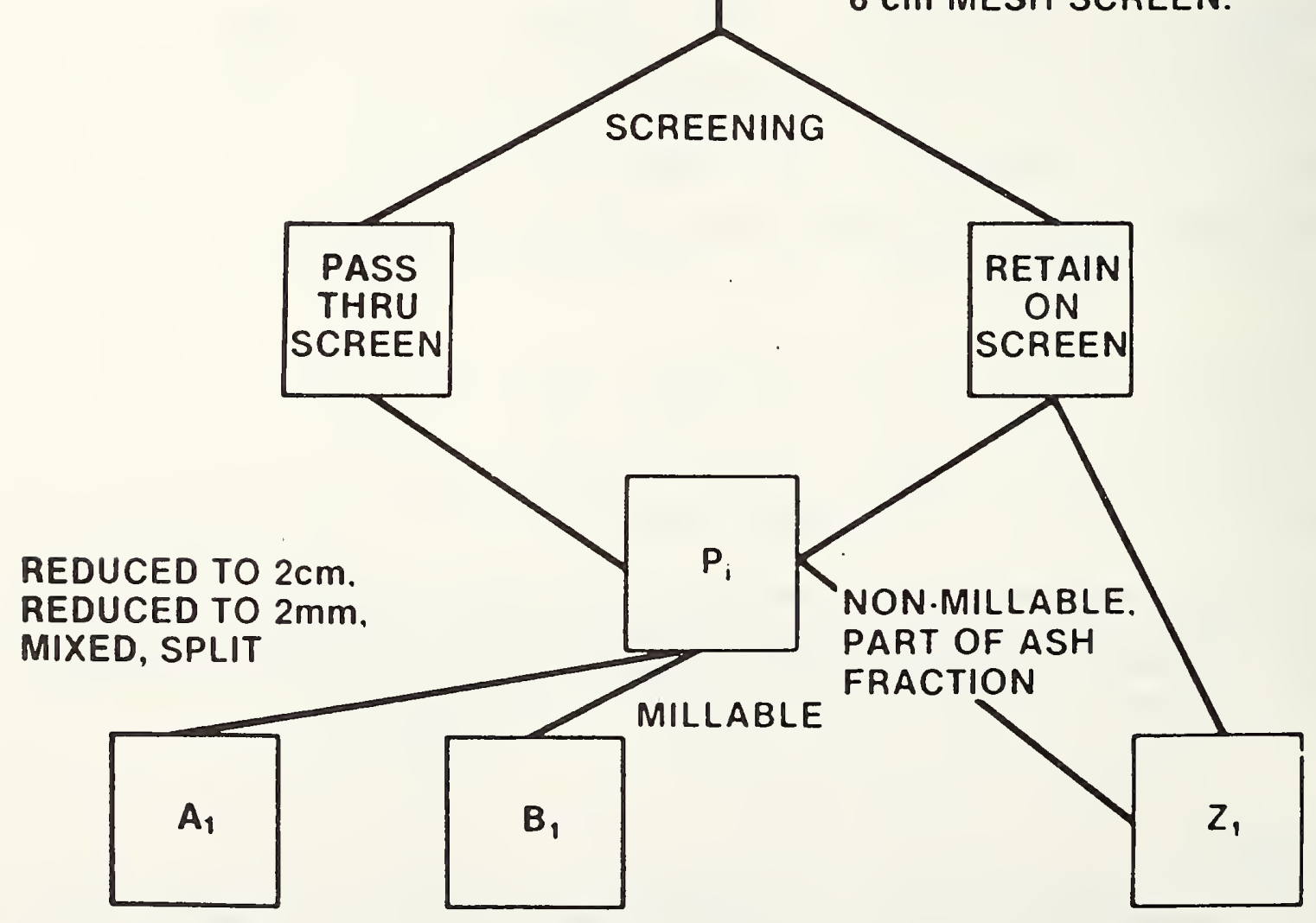

SAMPLE WAS WEIGHED.

DRIED AT $105^{\circ} \mathrm{C}$;

TOTAL MOISTURE

DETERMINED

SAMPLE EMPTIED ONTO $6 \mathrm{~cm}$ MESH SCREEN.

DETERMINATIONS: HHV, RESIDUAL MOISTURE. AND ASH

Fig. 1. RDF.2 Sample Processing Scheme 
The dry material from \#1 was passed through a 3 inch $(8 \mathrm{~cm})$ mesh screen and the weight of two fractions: PI (passing) and Zl (retained) were determined. Exceptions were made to the $\mathrm{Pl}$ fraction if a piece of non-millable material,such as if a long metal rod (say $2 \mathrm{~cm}$ diameter and maybe $30 \mathrm{~cm}$ in length), passed through the screen. Similarly, if a large piece of combustible material was retained on the screen for the $\mathrm{Zl}$ fraction, it was sub-divided and added to the PI fraction. This $P 1$ fraction was reduced in size to $19 \mathrm{~mm}$ or less by means of a 5 horsepower hammer mill and further reduced to $2 \mathrm{~mm}$ or less using a Model 4 Wiley laboratory mi11. The $2 \mathrm{~mm}$ material was mixed in a "vee"-blender, coned and quartered, and opposite quarters combined to give an $A 1$ and $B 1$ fraction. Hence, from the two daily samples, four sub-samples resulted: fractions $A 1$ and $B 1$ from bag 1 , and fractions $A 2$ and $B 2$ from bag 2, each weighing about one kilogram.

\subsection{Procedure}

Measurements of residual moisture, furance ash, bomb ash, and higher heating value were made on each of the sub-samples. ASTM standard methods, D3173 (moisture in the analysis sample) and D3174 (ash in the analysis sample), developed for coal and coke using gram-size amounts of material were used with the exception of a modified ashing procedure. The "furnace ash" was determined at $575{ }^{\circ} \mathrm{C}$ and the "bomb ash" was determined as the residue from the higher heating value determination.

The calorific value which is commonly called the higher heating value in many ASTM standards is defined as the heat produced by combustion of a unit quantity of solid or liquid fuel under specified conditions [2]. The unit quantity of fuel can be corrected for residual moisture and/or ash content to obtain the calorific value on a different basis. Although the as received MSW samples were dried, the hydroscopic cellulosic RDF regained moisture from the atmosphere. This quantity of moisture, which is in equilibrium with the ambient conditions, is the residual moisture. Ash is the non-combustible portion of RDF and was determined by two methods which gives rise to the terms "furnace ash" and "bomb ash." Quantities 
of about 2.5 each were extracted from the sub-samples and pressed into pellets for residual moisture, furnace ash, and higher heating value determinations. Bomb ash was determined from each calorimetric experiment by weighing the residue remaining in the combustion bomb.

This study involved 40 samples, 4 sub-samples for each day, from a two-week period. The determinations of the 20 A-sub-samples and $20 \mathrm{~B}$-sub-samples were randomized separately so that if a physicochemical process due to aging of the sample were taking place it might be detected more readily than if sub-samples from the first day were done first, those from the second day were done second, and so on. Measurements were made on the randomized A sub-samples followed by measurements on the randomized B sub-samples. In addition, any systematic errors due to the increased familiarity of the analyst with testing procedures, preparation of the samples, operation of the bomb calorineter, and the like, could be more readily apparent as a result of the randomization.

\section{Discussion of Results}

The results of the determinations for total moisture, residual moisture, metal fraction (non-millable materials), furnace ash, bomb ash, and higher heating value (as-determined) are shown in Table 2. The first column identifies the sample day, bag, and fraction. Columns 2 through 7 list data on residual moisture, total moisture, metal (non-millable) fraction, furnace ash, bomb ash, and higher heating value (on an as-determined basis), respectively. Although total moisture was determined during the sample processing procedures, the 40 sub-samples gained inoisture due to the hygroscopic nature of cellulosic RDF. Therefore, residual moisture relates to the increase in sample weight due to moisture pick-up after sample processing. The metal fraction, furnace ash, and bomb ash are reported on a dry basis. Table 3 lists data on total furnace ash (dry basis) and total bomb ash (dry basis) in columns 2 and 3 , respectively. The amount of non-millable material listed in Table 2 , column 4 was combined with the furnace ash or bomb 
ash from Table 2, columns 5 and 6 , respectively, to determine the total ash content of the as received MSW. Also in Table 3 are data on higher heating values which were calculated on different bases; HHV1 denotes the as-received basis; HHV2 is calculated to the dry basis, and HHV3 represents the dry-ash-free basis calculated from both furance and bomb ash data. Table 4 contains all of the higher heating values (i.e., calorific values) in the common USA engineering unit of Btu/lb. Calculation to different bases were performed by using the standard ASTM test procedures for coal [3].

Figs. 2-12 are histograms which show that the moisture content, ash content, non-millable material and higher heating values vary for each day, for each bag, and for each fraction of a bag. The display of the total moisture content is shown in Fig. 2 and shows a span of values of 16 wt. \% (day 7, bag 2) to 52 wt. \% (day 9, bag 1). The data in Fig. 3, which gives the residual moisture content of the samples, shows a variation from 1.4 to 4.1 wt. \%. The absorption of moisture back into the RDF samples remained within reasonably narrow limits and was dependent upon the constancy of the humidity in the laboratory, as well as the nature of the sample itself. The bomb ash and furnace ash values for the 10 day sampling period are shown in Figs. 4 and 5, respectively, ranging from about 10 to $49 \mathrm{wt} . \%$. The variation in the non-millable fraction over the 10 day sampling period is given in Fig. 6; it ranges from 1.6 to $20.6 \mathrm{wt} . \%$ and is less than the variability observed for either total moisture or total ash content. The total ash content is obtained by summing the quantities of non-millable materials with the corresponding data for either bomb ash or furnace ash; these data are given in Figs 7 and 8 , respectively. The total ash variability is comparable to the total moisture variability.

Data on the higher heating value on an as-received basis (HHVI) for the 10 day sampling period are shown in Fig. 9. The variability spans a range from $6.992 \mathrm{MJ} / \mathrm{kg}(3006 \mathrm{Btu} / \mathrm{lb}$ ) (day 9, bag 1, fraction B) to $14.144 \mathrm{MJ} / \mathrm{kg}(6081 \mathrm{Btu} / \mathrm{lg}$ ) 
Table 2. Test Data

\begin{tabular}{|c|c|c|c|c|c|c|}
\hline $\begin{array}{r}\text { Sample } \\
\text { Day/bag }\end{array}$ & $\begin{array}{l}\text { Resid. } \\
\text { Moist. } \\
\text { Wt. } \%\end{array}$ & $\begin{array}{l}\text { Total } \\
\text { Moist. } \\
\text { Wt. \% }\end{array}$ & $\begin{array}{l}\text { lietal } \\
\text { rract. } \\
\text { Wt. } \%\end{array}$ & $\begin{array}{c}\text { Bomb } \\
\text { Ash } \\
\text { Wt. \% }\end{array}$ & $\begin{array}{c}\text { Furnace } \\
\text { Ash } \\
\text { Wt. } \%\end{array}$ & $H H V-A D$ \\
\hline $\begin{array}{r}1 / 1 \mathrm{~A} \\
1 \mathrm{~B} \\
2 \mathrm{~A} \\
2 \mathrm{~B}\end{array}$ & $\begin{array}{l}2.01 \\
2.87 \\
2.56 \\
3.51\end{array}$ & $\begin{array}{l}24.72 \\
25.60\end{array}$ & $\begin{array}{l}1.63 \\
2.04\end{array}$ & $\begin{array}{l}36.05 \\
34.43 \\
19.78 \\
21.28\end{array}$ & $\begin{array}{l}42.62 \\
53.06 \\
19.28 \\
23.49\end{array}$ & $\begin{array}{l}13.930 \\
14.079 \\
16.777 \\
16.463\end{array}$ \\
\hline $\begin{array}{r}2 / 1 \mathrm{~A} \\
1 \mathrm{~B} \\
2 \mathrm{~A} \\
2 \mathrm{~B}\end{array}$ & $\begin{array}{l}2.76 \\
2.67 \\
2.99 \\
2.96\end{array}$ & $\begin{array}{l}24.96 \\
21.85\end{array}$ & $\begin{array}{l}4.17^{\circ} \\
3.49\end{array}$ & $\begin{array}{l}24.95 \\
24.56 \\
17.09 \\
16.86\end{array}$ & $\begin{array}{l}26.12 \\
29.93 \\
17.67 \\
22.32\end{array}$ & $\begin{array}{l}16.091 \\
16.166 \\
17.861 \\
18.199\end{array}$ \\
\hline $\begin{array}{r}3 / 1 \mathrm{~A} \\
1 \mathrm{~B} \\
2 \mathrm{~A} \\
2 \mathrm{~B}\end{array}$ & $\begin{array}{l}2.10 \\
2.53 \\
1.68 \\
2.56\end{array}$ & $\begin{array}{l}20.26 \\
21.02\end{array}$ & $\begin{array}{c}6.09 \\
11.28\end{array}$ & $\begin{array}{l}28.09 \\
32.88 \\
31.93 \\
31.87\end{array}$ & $\begin{array}{l}26.79 \\
35.47 \\
29.98 \\
37.14\end{array}$ & $\begin{array}{l}15.566 \\
14.896 \\
14.975 \\
14.605\end{array}$ \\
\hline $\begin{array}{r}4 / 1 A \\
1 B \\
2 A \\
2 B\end{array}$ & $\begin{array}{l}2.16 \\
2.17 \\
2.92 \\
2.50\end{array}$ & $\begin{array}{l}17.31 \\
17.77\end{array}$ & $\begin{array}{r}13.51 \\
7.30\end{array}$ & $\begin{array}{l}29.41 \\
39.52 \\
41.80 \\
46.93\end{array}$ & $\begin{array}{l}38.66 \\
41.96 \\
48.93 \\
53.69\end{array}$ & $\begin{array}{l}14.114 \\
12.914 \\
11.628 \\
10.786\end{array}$ \\
\hline $\begin{array}{r}5 / 1 A \\
1 B \\
2 A \\
2 B\end{array}$ & $\begin{array}{l}2.06 \\
3.18 \\
2.82 \\
3.48\end{array}$ & $\begin{array}{l}38.92 \\
44.70\end{array}$ & $\begin{array}{l}3.10 \\
3.97\end{array}$ & $\begin{array}{l}14.61 \\
20.73 \\
17.27 \\
17.96\end{array}$ & $\begin{array}{l}16.26 \\
22.45 \\
16.64 \\
19.11\end{array}$ & $\begin{array}{l}17.822 \\
16.761 \\
17.057 \\
16.845\end{array}$ \\
\hline $\begin{array}{r}6 / 1 A \\
1 B \\
2 A \\
2 B\end{array}$ & $\begin{array}{l}2.48 \\
2.84 \\
1.36 \\
2.58\end{array}$ & $\begin{array}{l}26.40 \\
27.78\end{array}$ & $\begin{array}{r}12.44 \\
8.98\end{array}$ & $\begin{array}{l}31.45 \\
44.64 \\
35.97 \\
34.07\end{array}$ & $\begin{array}{l}34.36 \\
40.12 \\
34.69 \\
35.91\end{array}$ & $\begin{array}{l}14.305 \\
12.170 \\
14.075 \\
14.175\end{array}$ \\
\hline $\begin{array}{r}7 / 1 \mathrm{~A} \\
1 \mathrm{~B} \\
2 \mathrm{~A} \\
2 \mathrm{~B}\end{array}$ & $\begin{array}{l}2.18 \\
2.06 \\
1.54 \\
2.85\end{array}$ & $\begin{array}{r}24.55 \\
16.08\end{array}$ & $\begin{array}{l}14.23 \\
20.63\end{array}$ & $\begin{array}{l}36.92 \\
44.50 \\
33.32 \\
33.68\end{array}$ & $\begin{array}{l}39.55 \\
42.06 \\
34.17 \\
34.83\end{array}$ & $\begin{array}{l}13.996 \therefore \\
12.530 \\
14.442 \\
14.172\end{array}$ \\
\hline $\begin{array}{r}8 / 1 \mathrm{~A} \\
1 \mathrm{~B} \\
(2 \mathrm{~A} \\
(2 \mathrm{~B}\end{array}$ & $\begin{array}{l}1.47 \\
2.41 \\
1.79 \\
2.57\end{array}$ & $\begin{array}{l}20.68 \\
21.16\end{array}$ & $\begin{array}{l}7.03 \\
3.52\end{array}$ & $\begin{array}{l}43.20 \\
41.41 \\
59.38 \\
37.47\end{array}$ & $\begin{array}{l}43.49 \\
45.90 \\
48.46 \\
39.97\end{array}$ & $\begin{array}{c}12.660 \\
12.767 \\
9.053) \\
13.049)\end{array}$ \\
\hline $\begin{array}{r}9 / 1 A \\
1 B \\
2 A \\
2 B\end{array}$ & $\begin{array}{l}2.40 \\
3.43 \\
3.08 \\
2.93\end{array}$ & $\begin{array}{l}52.03 \\
23.33\end{array}$ & $\begin{array}{l}4.71 \\
2.67\end{array}$ & $\begin{array}{l}23.59 \\
27.96 \\
24.84 \\
32.94\end{array}$ & $\begin{array}{l}23.42 \\
28.90 \\
26.60 \\
33.47\end{array}$ & $\begin{array}{l}15.647 \\
14.770 \\
15.435 \\
14.058\end{array}$ \\
\hline $\begin{array}{r}10 / 1 A \\
1 B \\
2 A \\
2 B\end{array}$ & $\begin{array}{l}1.95 \\
3.05 \\
2.77 \\
4.13\end{array}$ & 22.67 & $\begin{array}{l}3.90 \\
4.64\end{array}$ & $\begin{array}{l}9.66 \\
9.59 \\
9.93 \\
9.82\end{array}$ & $\begin{array}{l}10.98 \\
10.18 \\
13.76 \\
14.14\end{array}$ & $\begin{array}{l}17.982 \\
17.813 \\
16.994 \\
16.403\end{array}$ \\
\hline
\end{tabular}


Sample Total Ash

Day/bag Furnace Bomb wit. $\%$ wt.

$1 / 1 \mathrm{~A}$

$1 B$

$2 A$

$2 B$

$2 / 1 \mathrm{~A}$

$1 \mathrm{~B}$

$2 \mathrm{~A}$

2B

$3 / 1 \mathrm{~A}$

$1 \mathrm{~B}$

$2 \mathrm{~A}$

2B

$4 / 1 \mathrm{~A}$

$1 B$

$2 A$

$2 B$

$5 / 1 \mathrm{~A}$

$1 B$

$2 \mathrm{~A}$

$2 B$

$6 / 1 A$

$1 B$

$2 \mathrm{~A}$

2B

$7 / 1 \mathrm{~A}$

$1 B$

$2 \mathrm{~A}$

$2 B$

$8 / 1 A$

$1 \mathrm{~B}$

$(2 \mathrm{~A}$

(2B

9/1A

$1 \mathrm{~B}$

$2 \mathrm{~A}$

2B

$10 / 1 \mathrm{~A}$

$1 \mathrm{~B}$

$2 A$

$2 B$
43.55

53.83

20.93

25.04

29.20

32.86

20.54

25.03

31.25

39.40

37.88

44.23

46.94

49.80

52.66

57.07

18.85

24.86

19.95

22.33

42.53

47.57

40.56

41.66

48.15

50.31

47.75

48.28

47.46

49.71

50.27

42.08

27.03

32.25

28.56

35.25

14.46

13.68

17.76

18.13
37.09

35.50

21.41

22.88

28.03

27.70

19.98

19.77

32.47

36.97

39.61

39.56

38.94

47.69

46.05

50.81

17.26

23. 18

20.55

21.22

$39.98^{\circ}$

51.53

41.72

39.99

45.89

52.40

47.08

47.36

47.19

45.53

60.81

39.69

27.18

31.35

26.84

34.73

13.18

13.12

14.10

$14: 00$
HHV - 1

$\mathrm{MJ} / \mathrm{kg}$

$\begin{array}{ll}10.527 & 14.217 \\ 10.734 & 14.496 \\ 12.549 & 17.217 \\ 12.435 & 17.064\end{array}$

11.900

11.944

13.886

14.144

11.907

11.444

10.672

10.502

10.318
9.441
9.130
8.432

16.547

16.610

18.410

18.755

15.901

15.282

15.231

14.989

14.426

13.200

11.977

11.062

10.769

10.246

9.320

9.267

9.453

8.071

9. 381

9.565

9.260

8. 278

9.769

9.716

18.196

17.312

17.552

17.452

14.668

12.526

14.268

14.549

9.476

9.648

7.011

10.188

14.307

12.793

14.668

14.589

12.849

13.084

9.218

13.393

7.329

6.992

11.884

10.807

13. 156

13.179

12.888

12.616
16.031

15.296

15.926

14.484

18. 341

18.373

17.478

17.110 \begin{tabular}{cc}
\multicolumn{2}{c}{$H \mathrm{HV}-3$} \\
Furnace & Bomb \\
$\mathrm{MJ} / \mathrm{kg}$ & $\mathrm{MJ} / \mathrm{kg}$
\end{tabular}

$\begin{array}{ll}24.774 & 22.232 \\ 31.931 & 22.106 \\ 21.332 & 21.462 \\ 22.299 & 21.674\end{array}$

$22.397 \quad 22.048$

$23.704 \quad 22.016$

$22.362 \quad 22.206$

$24.142 \quad 22.558$

$21.718 \quad 22.111$

$23.681 \quad 22.769$

$21.753 \quad 22.374$

$23.844 \quad 21.9 .99$

$23.516 \quad 20.434$

$22.741 \quad 21.827$

$23.453 \quad 20.580$

$23.888 \quad 20.846$

$21.729 \quad 21.311$

$22.325 \quad 21.839$

$21.055 \quad 21.215$

$21.576 \quad 21.274$

$22.348 \quad 21.399$

$20.918 \quad 22.627$

$21.848 \quad 22.283$

$21.702 \quad 22.067$

$23.669 \quad 22.679$

$22.081 \quad 23.053$

$22.281 \quad 21.999$

$22.385 \quad 21.997$

$22.739 \quad 22.620$

$24.183 \quad 22.330$

17.885 22.695)

$22.309 \quad 21.420)$

$20.934 \quad 20.981$

$21.513 \quad 21.232$

$21.697 \quad 21.188$

$21.769 \quad 21.597$

$20.604 \quad 20.301$

$20.453 \quad 20.322$

$20.266 \quad 19.403$

$19.929 \quad 18.971$ 


\begin{tabular}{|c|c|c|c|c|c|}
\hline \multirow{2}{*}{$\begin{array}{r}\text { Sample } \\
\text { Day/bag }\end{array}$} & \multirow[t]{2}{*}{$H H V-A D$} & \multirow[t]{2}{*}{$H H V-1$} & \multirow[t]{2}{*}{$\mathrm{HHV}-2$} & \multicolumn{2}{|c|}{$\mathrm{HHV}-3$} \\
\hline & & & & Furnace & Bomb \\
\hline & $\mathrm{BTU} / \mathrm{Ib}$ & $\mathrm{BTU} / \mathrm{Ib}$ & $\mathrm{BTU} / \mathrm{Ib}$ & $\mathrm{BTU} / \mathrm{Ib}$ & $\mathrm{BTU} / \mathrm{Ib}$ \\
\hline $1 / 1 \mathrm{~A}$ & 5989 & 4526 & 6112 & 10651 & 9558 \\
\hline IB & 6053 & 4615 & 6232 & 13728 & 9504 \\
\hline $2 \mathrm{~A}$ & 7213 & 5395 & 7402 & 9171 & 9227 \\
\hline $2 B$ & 7078 & 5346 & 7336 & 9587 & 9318 \\
\hline $2 / 1 \mathrm{~A}$ & 6918 & 5116 & 7114 & 9629 & 9479 \\
\hline $1 \mathrm{~B}$ & 6950 & 5135 & 7141 & 10191 & 9465 \\
\hline $2 \mathrm{~A}$ & 7679 & 5970 & 7915 & 9614 & 9547 \\
\hline $2 B$ & 7824 & 6081 & 8063 & 10379 & 9698 \\
\hline $3 / 1 \mathrm{~A}$ & 6692 & 5119 & 6836 & 9337 & 9506 \\
\hline $1 \mathrm{~B}$ & 6404 & 4920 & 6570 & 10181 & 9789 \\
\hline $2 \mathrm{~A}$ & 6438 & 4588 & 6548 & 9352 & 9619 \\
\hline $2 B$ & 6279 & 4515 & 6444 & 10251 & 9458 \\
\hline $4 / 1 \mathrm{~A}$ & 6068 & 4436 & 6202 & 10110 & 8785 \\
\hline $1 \mathrm{~B}$ & 5552 & 4059 & 5675 & 9777 & 9384 \\
\hline $2 \mathrm{~A}$ & 4999 & 3925 & 5149 & 10083 & 8848 \\
\hline $2 B$ & 4637 & 3625 & 4756 & 10270 & 8962 \\
\hline $5 / 1 \mathrm{~A}$ & 7662 & 4630 & 7823 & 9342 & 9162 \\
\hline & 7206 & 4405 & 7443 & 9598 & 9389. \\
\hline $2 \mathrm{~A}$ & 7333 & 4007 & 7546 & 9052 & $9121^{\circ}$ \\
\hline $2 B$ & 7242 & 3984 & 7503 & 9276 & 9146 \\
\hline $6 / 1 \mathrm{~A}$ & ' 6150 & 4064 & 6306 . & 9608 & 9200 \\
\hline IB & 5232 & 3470 & $5385^{\circ}$ & 8993 & 9728 \\
\hline $2 A$ & 6051 & 4033 & 6134 & 9393 & 9580 \\
\hline $2 B$ & 6094 & 4112 & 6255 & 9760 & 9487 \\
\hline $7 / 1 \mathrm{~A}$ & 6017 & 3981 & 6151 & 10176 & 9750 \\
\hline 1B & 5387 & 3559 & 5500 & 9493 & 9911 \\
\hline $2 \mathrm{~A}$ & 6209 & 4200 & 6306 & 9579 & 9458 \\
\hline $2 B$ & 6093 & 4177 & 6272 & 9624 & 9457 \\
\hline $8 / 1 \mathrm{~A}$ & 5443 & 4074 & 5524 & 9776 & 9725 \\
\hline IB & 5489 & 4148 & 5625 & 10397 & 9600 \\
\hline$(2 \mathrm{~A}$ & 3892 & 3014 & 3963 & 7689 & 9757) \\
\hline$(2 B$ & 5610 & 4380 & 5758 & 9591 & 9209) \\
\hline $9 / 1 \mathrm{~A}$ & 6727 & 3151 & 6892 & 9000 & 9020 \\
\hline $1 B$ & 6350 & 3006 & 6576 & 9249 & 9128 \\
\hline $2 \mathrm{~A}$ & 6636 & 5109 & 6847 & 9328 & 9109 \\
\hline $2 \mathrm{~B}$ & 6044 & 4646 & 6227 & 9359 & 9285 \\
\hline $10 / 1 \mathrm{~A}$ & & 5656 & 7885 & 8858 & 8728 \\
\hline $1 \mathrm{~B}$ & 7658 & 5666 & 7899 & 8793 & 8737 \\
\hline $2 \mathrm{~A}$ & 7306 & 5541 & 7514 & 8713 & 8342 \\
\hline $2 B$ & 7052 & 5424 & 7356 & 8568 & 8156 \\
\hline
\end{tabular}


(day 2, bag 2, fraction $\mathrm{B}$ ). The range is over $6.978 \mathrm{MJ} / \mathrm{kg}(3000 \mathrm{Btu} / \mathrm{lb})$. In Fig. 10, data on the higher heating value on a dry basis (HHV2) are reported for the 10 day sampling period. The high and low values are $18.755 \mathrm{MJ} / \mathrm{kg}$ $(8063 \mathrm{Btu} / \mathrm{lb})$ and $9.218 \mathrm{MJ} / \mathrm{kg}(3963 \mathrm{Btu} / \mathrm{lb})$. In this case, the range is more than $9.304 \mathrm{MJ} / \mathrm{kg}(4000 \mathrm{Btu} / \mathrm{lb})$. The data on the higher heating values on a dryash-free basis (HHV3) are given in two forms. These are related to the furnace ash and bomb ash data, and are given in Figs. 11 and 12, respectively. The data for HHV3 based on the furnace ash have a high and low value of $30.885 \mathrm{MJ} / \mathrm{kg}$ (13278 Btu/1b) and $19.929 \mathrm{MJ} / \mathrm{kg}(8568 \mathrm{Btu} / \mathrm{lb})$ respectively, and corresponds to a range of $10.955 \mathrm{MJ} / \mathrm{kg}(4710 \mathrm{Btu} / \mathrm{lb})$. In comparison, the data for HHV3 based upon the bomb ash has a high and low value of $23.053 \mathrm{MJ} / \mathrm{kg}(9911 \mathrm{Btu} / \mathrm{lb})$ and $18.971 \mathrm{MJ} / \mathrm{kg}(8156 \mathrm{Btu} / \mathrm{lb})$ respectively. These data show a range of only $4.082 \mathrm{MJ} / \mathrm{kg}$ $(1755 \mathrm{Btu} / \mathrm{lb})$ and indicate that a closer correspondence exists between the HHV3 data and the bornb ash than the furnace ash since the former is formed directly as a result of burning the RDF sample in the bomb calorimeter.

The above discussion dealt solely with the variability indicated by the histograms. However, included in this variability is the within bag variability. The differences between the data for fraction $A$ and fraction $B$ has been examined for furnace ash, bomb ash, and are shown in figs. 13-15, respectively. The A-B difference for HHV2 appears to be mostly negative, while for both sets of ash data, the A-B difference is mostly positive. A systematic shift has taken place between the two sets of data and a reasonable explanation for it is not apparent.

The A-B differences for HHV2 and ash were also examined to determine the relationship between ash content data and calorific value data. This relationship is important because the ash values on a dry basis are combined with HHV2 on a dry basis to calculate HHV3 on a dry-ash-free basis. The existing ASTM standard procedures for ash determination [4] are done on a twin or equivalent analysis sample and the above results on fraction $A$ and fraction $B$ 
from within the same bag clearly show the inability to pick equivalent analysis samples from the same bag. Therefore, the A-B differences for HHV2 were plotted versus the $A-B$ differences for furnace ash and bomb ash and are shown in Figs. 16 and 17, respectively. Note that the furnace ash is determined on an equivalent (twin) sample and thus should show little or no correlation; the bomb ash is determined as the bomb residue from the actual calorific value measurement and thus should show a definite correlation. Figs. 16 and 17 verify this.

A definite inverse relationship exists between the A-B differences for HHV2 and bomb ash. This is not the case for HHV2 and the furnace ash. A correlation coefficient equal to -0.965 is obtained between the bomb ash and HHV2 showing a strong relationship while a value of -0.236 is found between the furnace ash and HHV2 indicating a much weaker and almost insignificant relationship.

During the course of examining the experimental data for HHV2, furnace ash and bomb ash, the A-B difference obtained for day 8, bag 2 appeared to be significantly large and atypical. For this reason, the data obtained for day 8 , bag 2 were omitted from figures 16 and 17 and from all statistical calculations.

\section{Statistical Analysis of Results}

A statistical analysis was performed on measurements determined for each of the following properties: (1) total moisture, (2) metal fraction, (3) furnace ash, (4) bomb ash, (5) HHV1, the higher heating value on an as-received basis, (6) HHV2, the higher heating value on a dry basis, (7) HHV3-F, the higher heating value on a dry and ash-free basis using furnace ash data, and (8) HHV3-B, the higher heating value on a dry ash-free basis using bomb data. Table 5 gives the statistical parameters for this study. The following set of statistical parameters were calculated for each set of measurements:

(1) average value shown in column 2;

(2) component of variability due to within bag variability shown in column 3; 
(3) component of variability due to within day variability shown in column 4;

(4) component of variability due to day to day variability shown in column 5; and

(5) the total variability in the last column on the right.

Table 5. Summary of Results

Components, expressed as \%CV for

\begin{tabular}{lccccc} 
Property & Average & $\begin{array}{c}\text { Within } \\
\text { Bag }\end{array}$ & $\begin{array}{c}\text { Bags } \\
\text { Within days }\end{array}$ & $\begin{array}{c}\text { Between } \\
\text { Days }\end{array}$ & Total $^{(a)}$ \\
\hline Total Moisture(\%) & 26.0 & -- & 27.9 & 22.7 & 36.0 \\
Metal Fraction(\%) & 7.2 & -- & 36.8 & 61.3 & 71.5 \\
Furnace Ash (\%) & 31.2 & 6.5 & 23.0 & 29.8 & 38.2 \\
Bomb Ash (\%) & 28.9 & 10.6 & 15.6 & 33.7 & 38.6 \\
HHV1 & 10.530 & 3.2 & 12.2 & 11.6 & 17.7 \\
HHV2 & 15.393 & 3.2 & 6.6 & 11.2 & 13.4 \\
HHV3-F & 22.523 & 5.1 & 5.4 & 3.1 & 8.0 \\
HHV3-B & 21.664 & 1.6 & 1.5 & 3.7 & 4.3 \\
\hline
\end{tabular}

(a) Total \%CV is the sum in quadrature of the \%CV for within bag, bags within days and between days. 
The statistical parameters expressed by items (2) through (5) are expressed as the percent coefficient of variation (\% CV) which is defined as \% CV $=(100)$ $S / \bar{X}$ where $S$ is the standard deviation of an individual measurement and $\bar{X}$ is the average or mean value.

The variabilities of the combustible and non-combustible components of municipal solid waste (MSW) were derived from this study. $\%$ CV in excess of $36 \%$ were calculated for the moisture and ash content of MSW. Between 70 and $80 \%$ of the total $\% \mathrm{CV}$ is from the within day and between day components of \% CV. The $\% \mathrm{CV}$ for HHV1 was 17.1. This indicates that variability of MSW is primarily due to moisture and ash content and not to combustible content. The variability of the combustible fraction of MSW can be extracted by looking at HHV3-B which is the higher heating value on a dry and bomb ash-free basis. The combustible fraction was and should be determined from HHV3-B rather than HHV3-F because the correlation coefficient, shown in the last section, between HHV2 and bomb ash was calculated to be -0.965 compared to a correlation coefficient of -0.236 between HHV2 and the furnace ash. The total \% CV for HHV-3 is only 4.3 which indicates that the combustible fraction of MSW is surprisingly homogeneous. In fact, the within bag variability accounts for $37.21 \%$ (1.6 of 4.3) of the total \% CV for HHV3-B and includes the $A-B$ differences which are most probably due to segregation of light and heavy particles, and are a reflection of the analyst's inability to choose equivalent analysis samples from the same bag.

5. Conclusions

(1) Data derived from carrying out test methods for moisture, ash, and calorific value show that the within day and between day variability of refuse are large and depend upon the nature of the waste stream. These variabilities constitute about 70 to $80 \%$ of the overall uncertainty in the measured properties.

(2) Test data suggest that improvements in the test method which impact upon attaining greater within bag precision will probably not increase the over- 
all precision of the measured properties in a significant manner.

Within bag variability constitute about 20 to $30 \%$ of the overall uncertainty.

(3) Data on the calorific value of RDF-2 can be expressed in a more precise manner using the bomb ash data because the actual ash which formed during the combustion calorimetric measurement is used to calculate the higher heating value on a moisture ash-free basis, HHV3-B.

(4) Total moisture, metal fraction, and ash content data show large variability on a within-day and between-day basis.

(5) The precision of data for the higher heating value improves as correction is made to remove contributions due to moisture and ash content. The overall coefficients of variability for HHV3-F and HHV3-B are 8.0 and $4.3 \%$, respectively. The experimental data indicate that HHV3-B is the most precise measure of the heating value of RDF, on a moisture and ash free basis.

(6) The data for HHV3-B shows that the combustible fraction of RDF is reasonably constant.

6. Acknowledgements

This research was funded by the NBS Office of Recycled Materials and the DoE Office of Energy from Municipal Waste.

We acknowledge the cooperation and assistance provided by the Baltimore County Resource Recovery Facility which is operated by the Teledyne National Corporation in Cockeysville, Maryland.

\section{References}

[1] ASTM Committee E38.93 on Terminology. Thesaurus compiled by Herbert I. Hollander, Sanders \& Thomas, Inc., Pottstown, Pa. 19464.

[2] ASTM Standard D121, Definition of Terms Relating to Coal and Coke.

[3] ASTM Standard D3174, Ash in the Analys is Sample of Coal and Coke.

[4] ASTM Standard D3180, Calculating Coal and Coke Analysis from As-Determined to Determined Bases. 


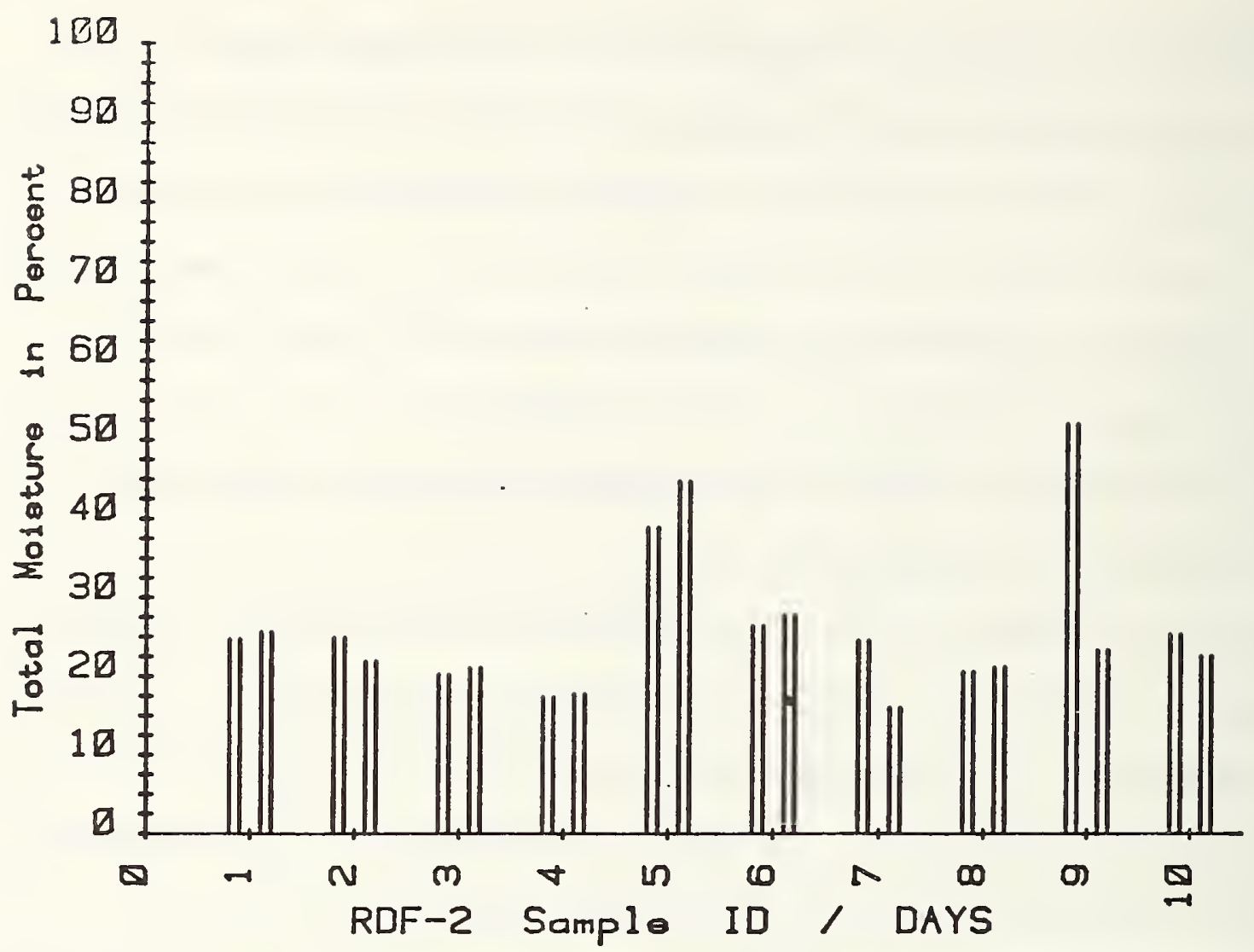

Fig. 2 Variability of Total Moisture

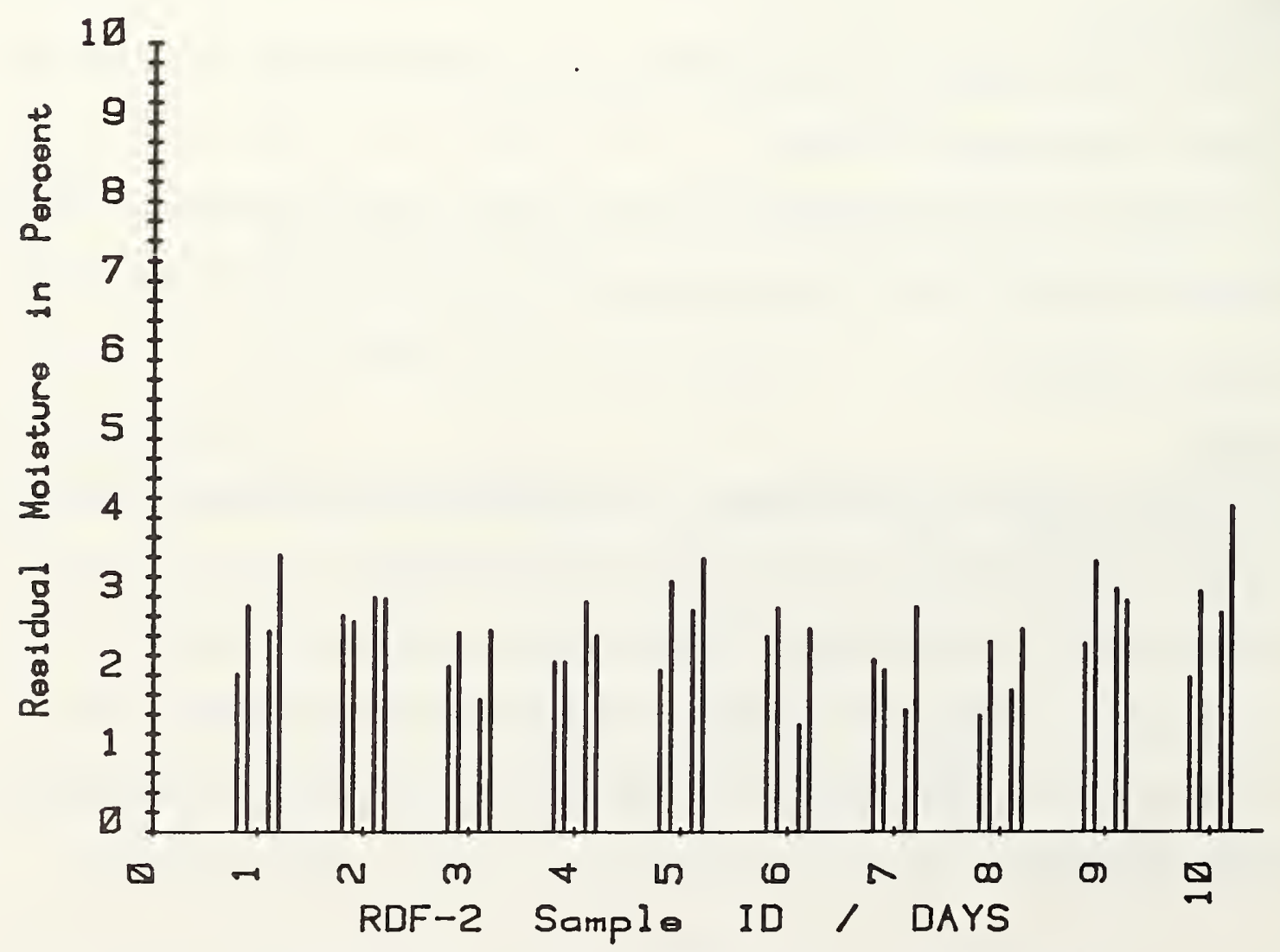

Fig. 3 Variability of Residual Moisture 


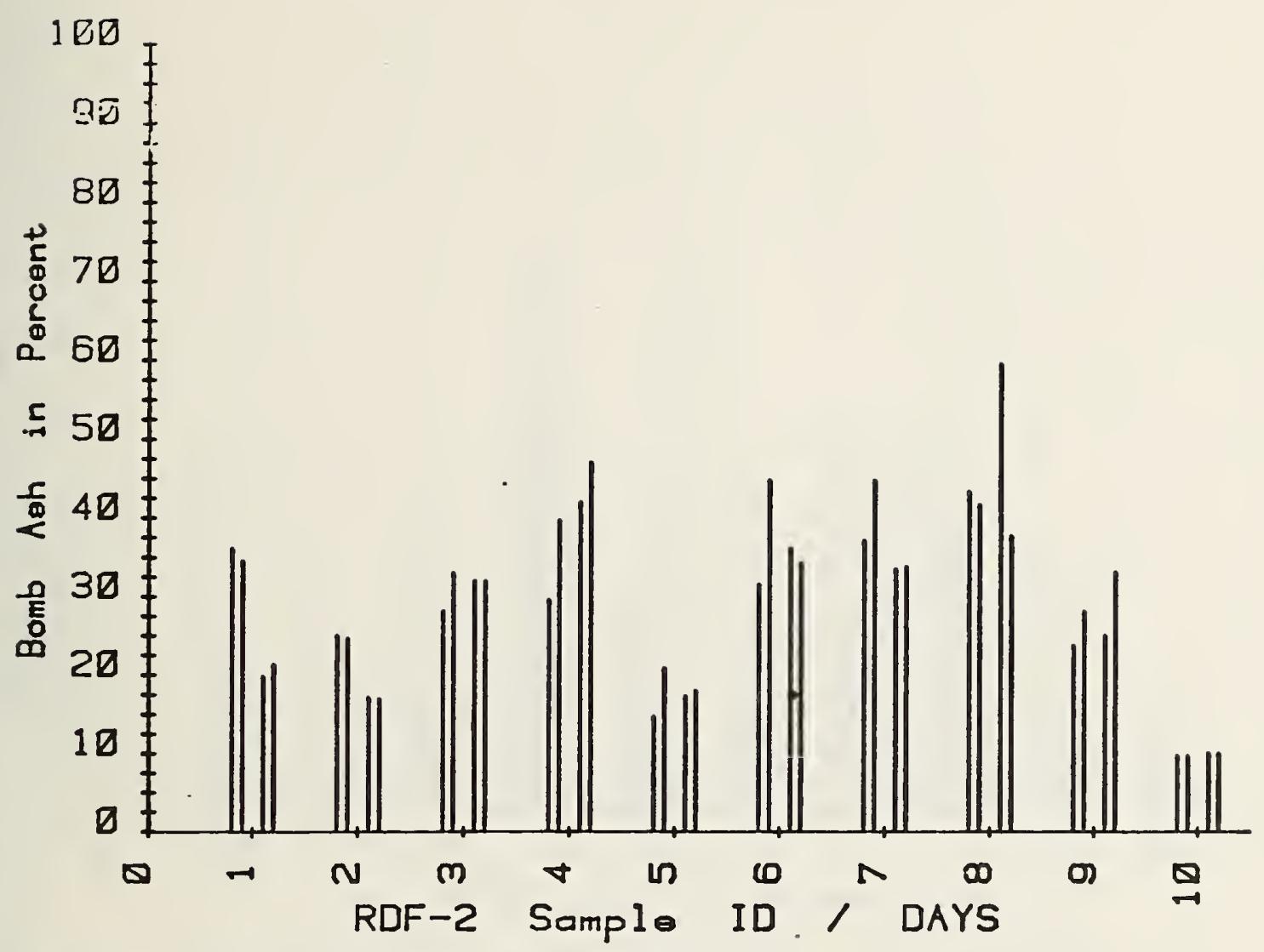

Fig. 4 Variability of Bomb Ash

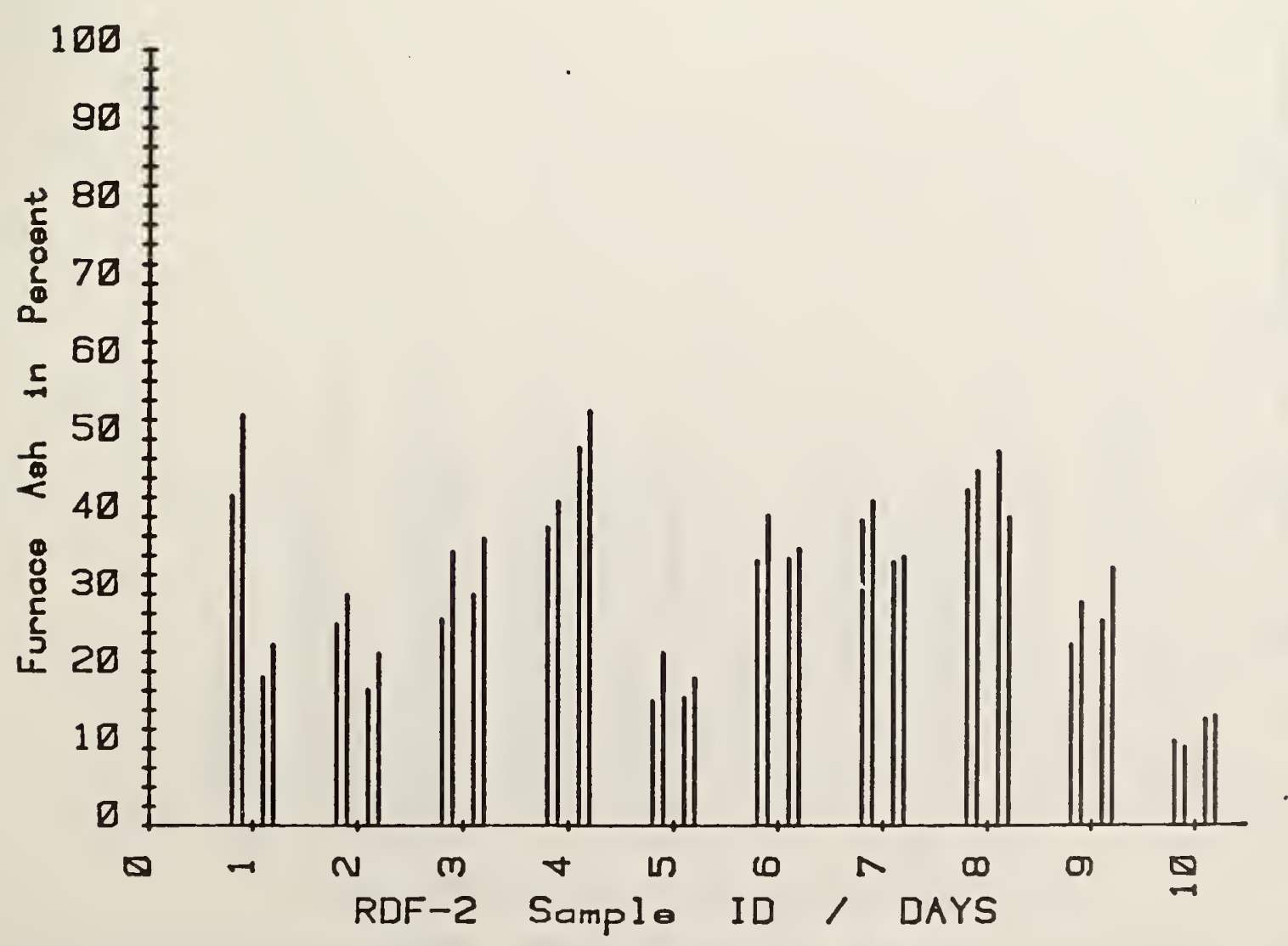

Fig. 5 Variability of Furnace Ash 


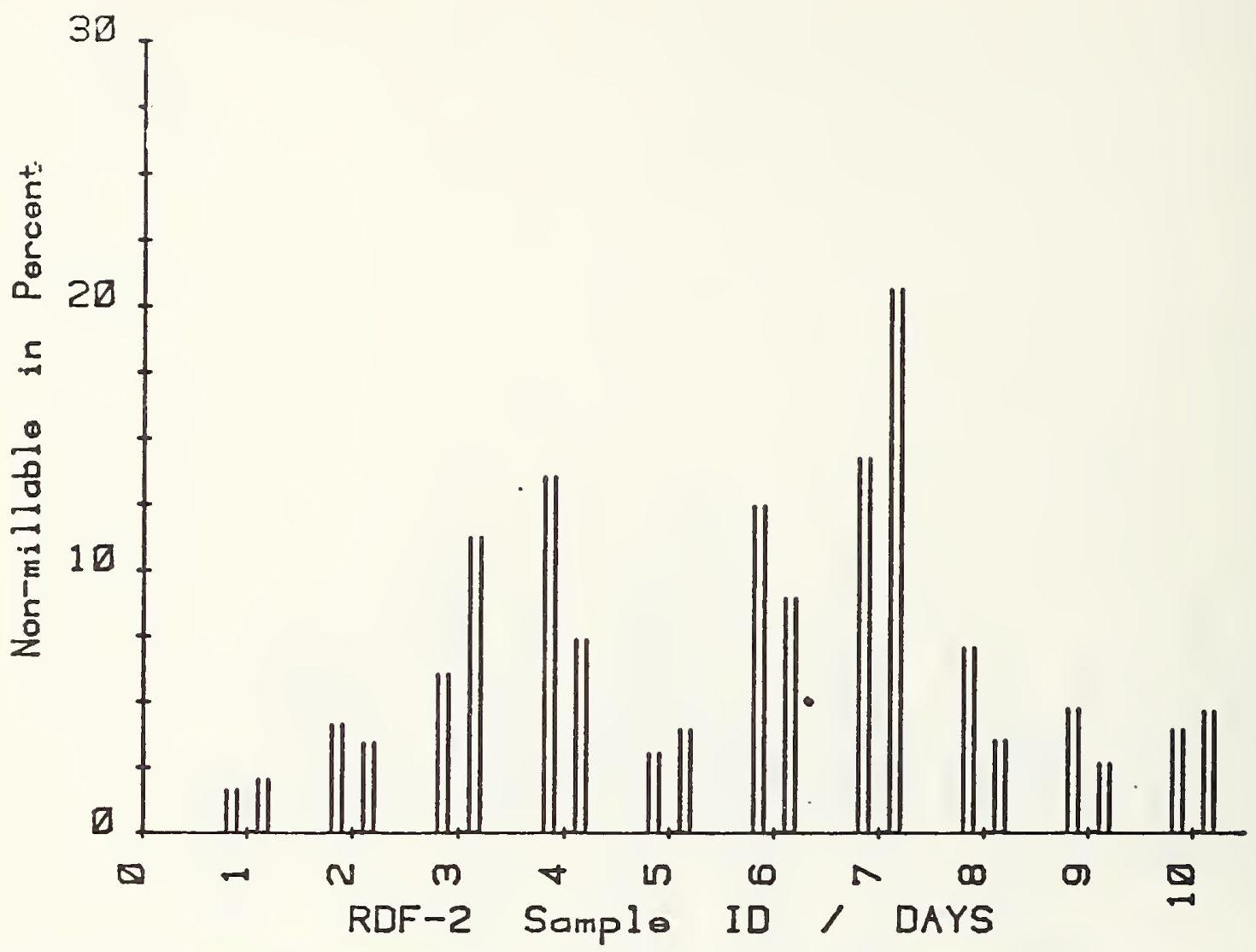

Fig. 6 Variability of Non-millable Material

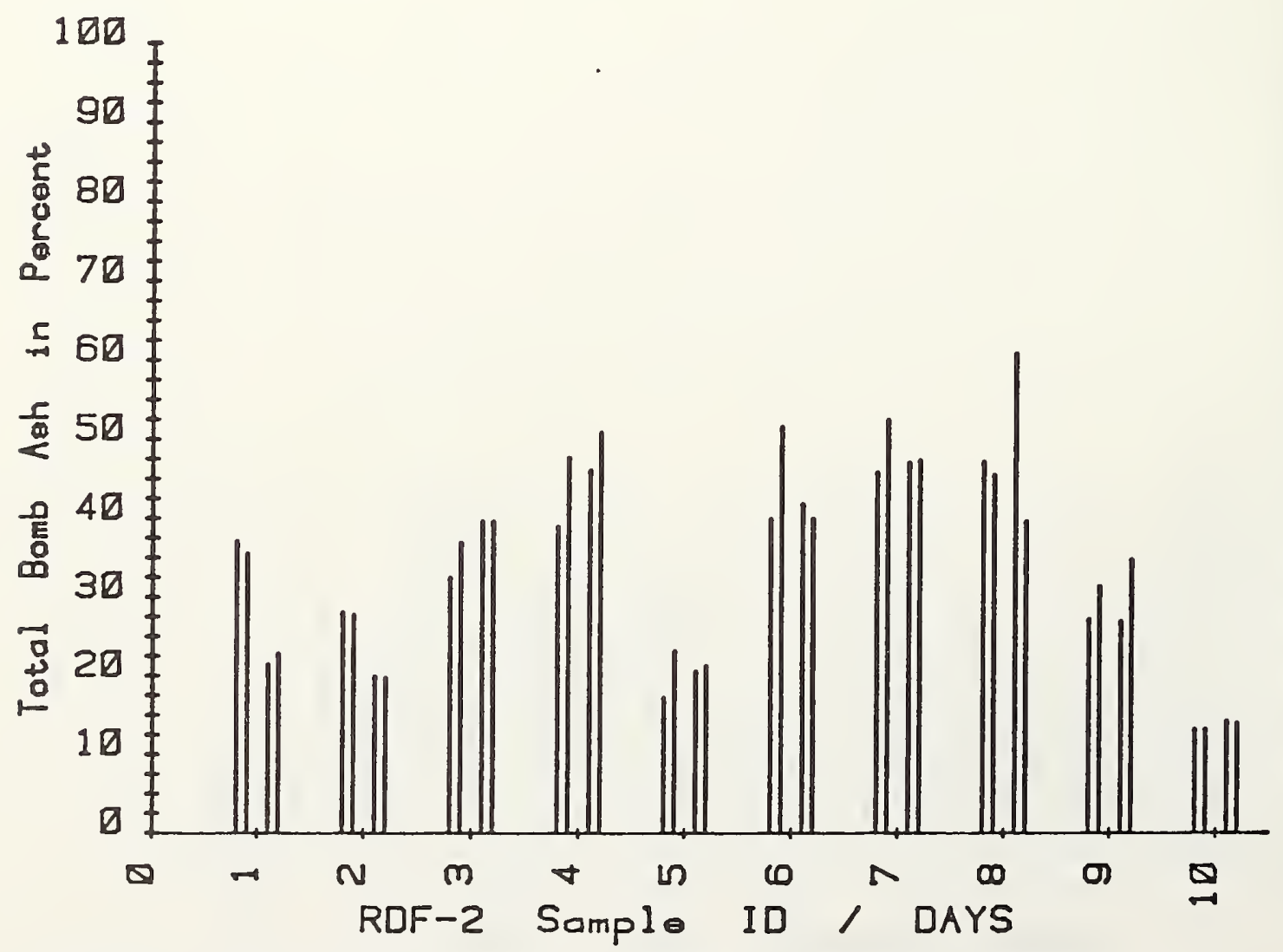

Fig. 7 Variability of Total Bomb Ash 


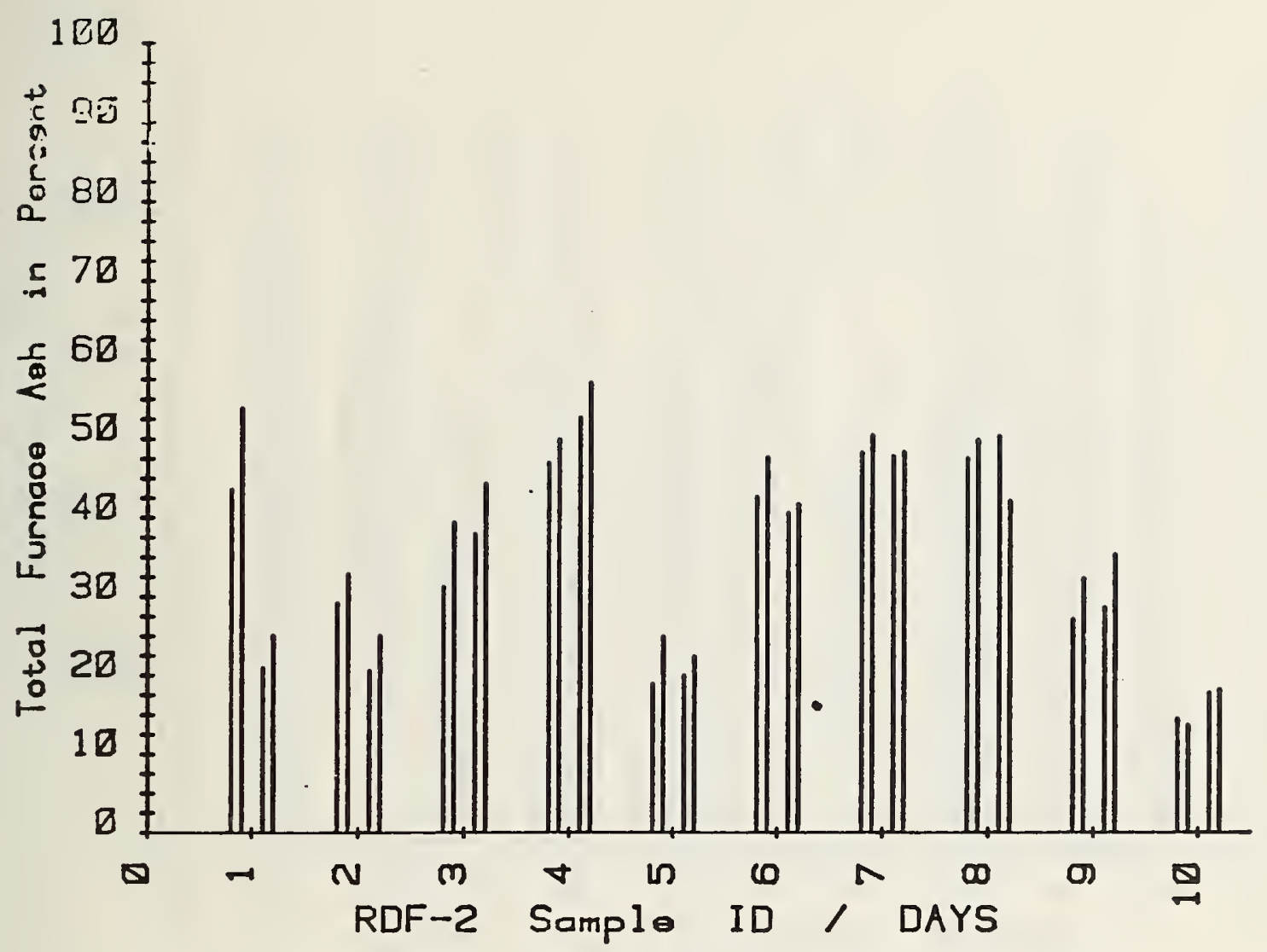

Fig. 8 Variability of Total Furnace Ash

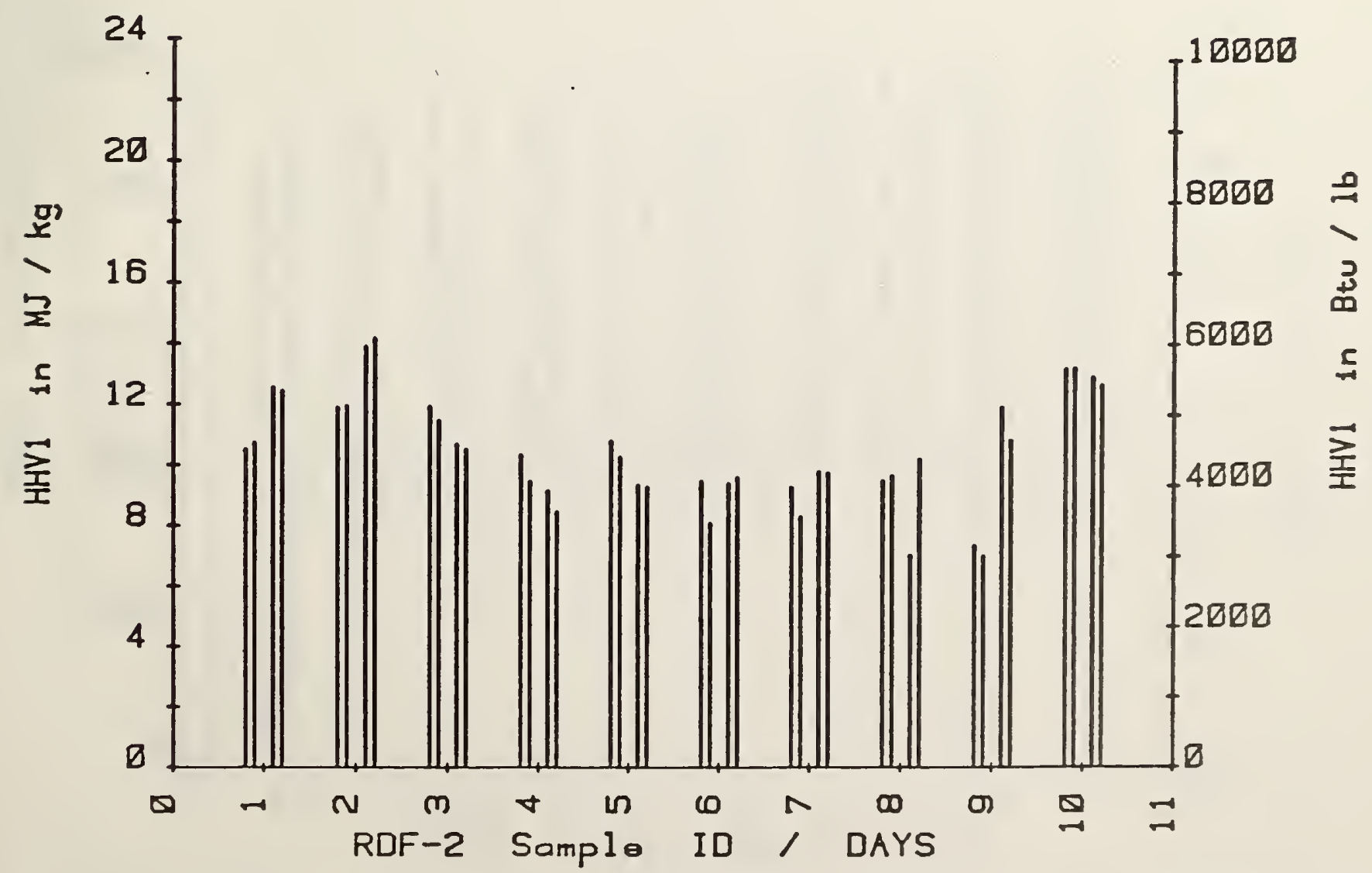

Fig. 9 Variability of HHVI 


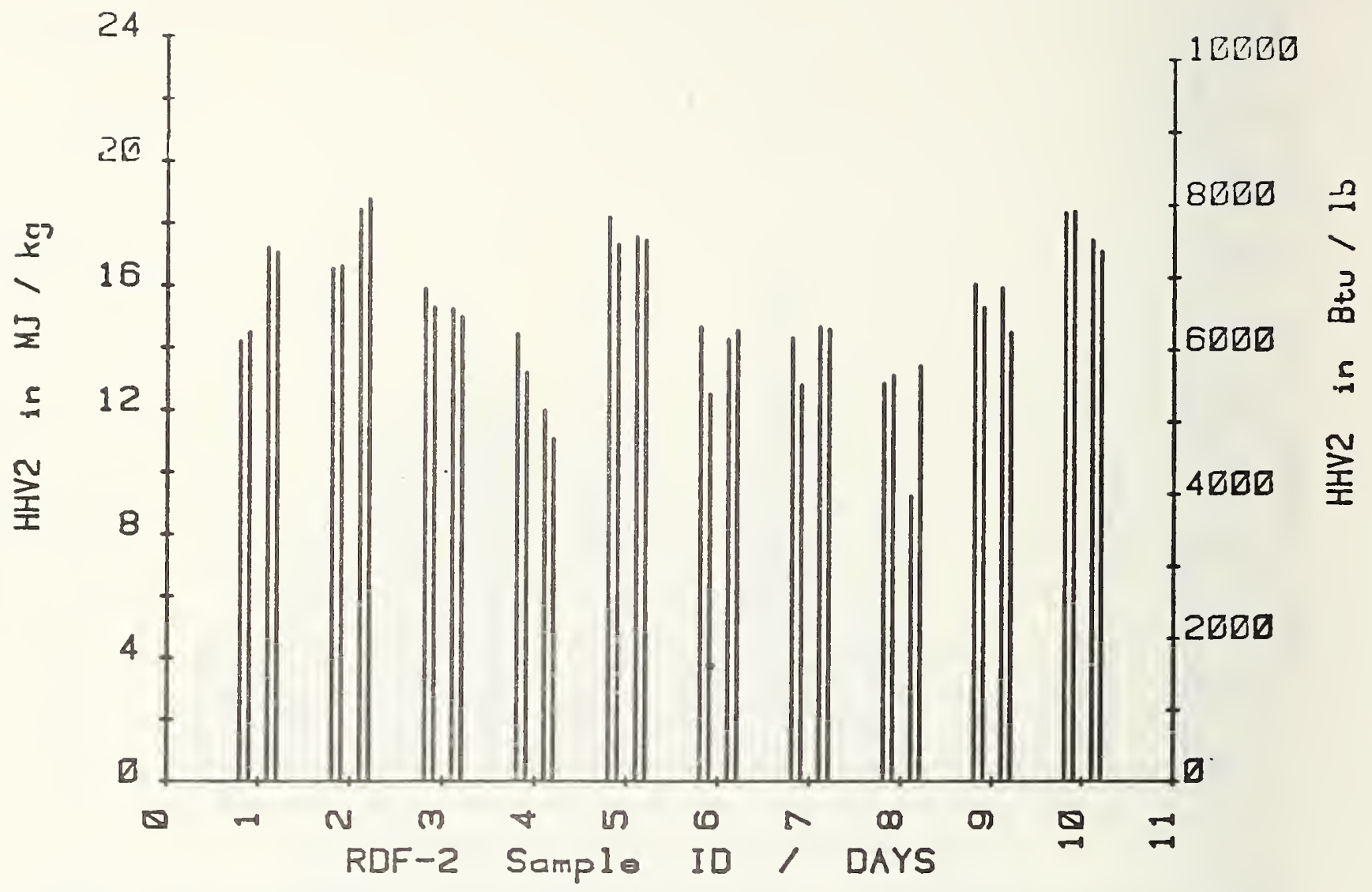

Fige 10 Variability of HHVZ

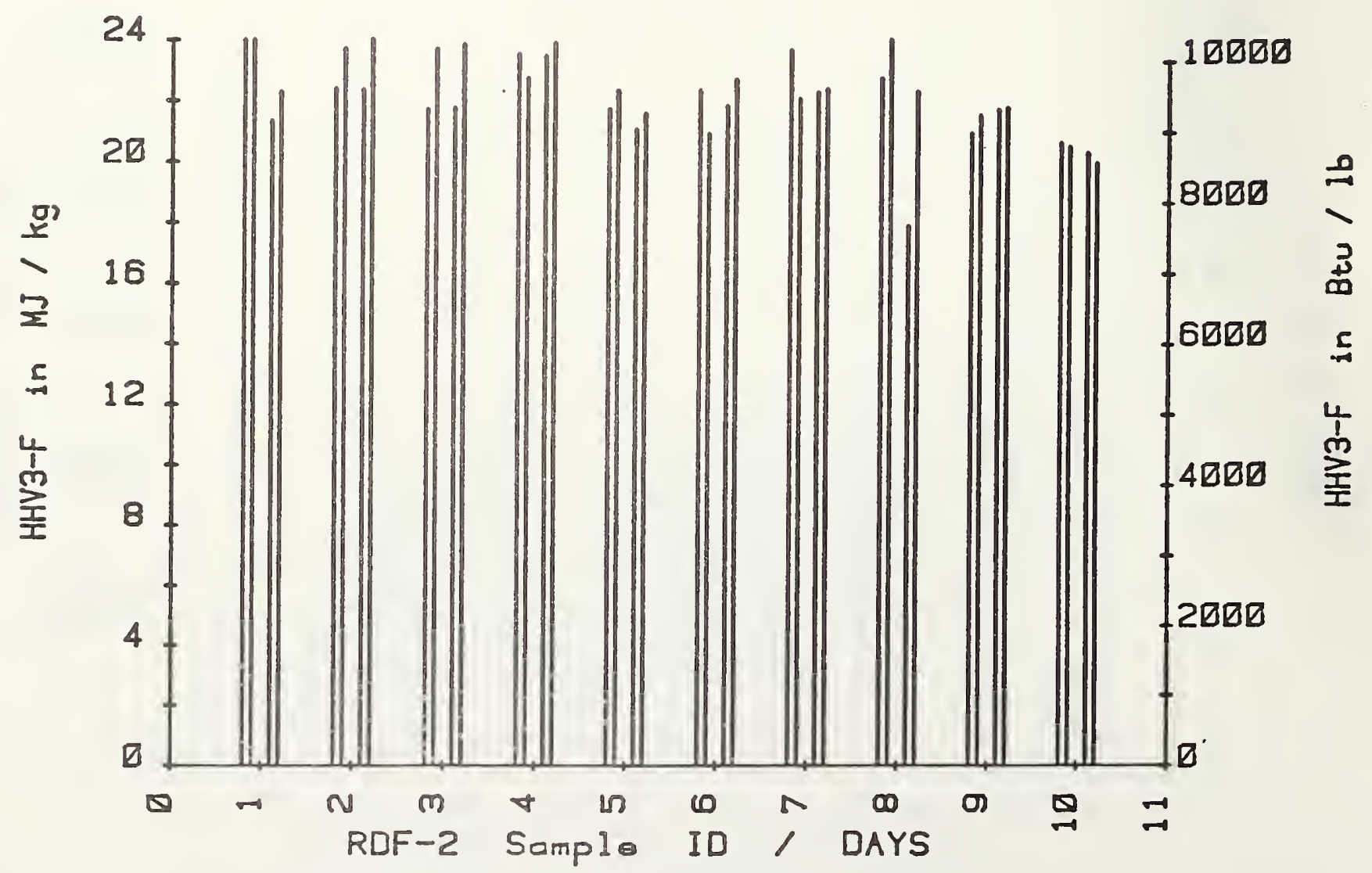

Fig. 11 Variability of HHV3-F 


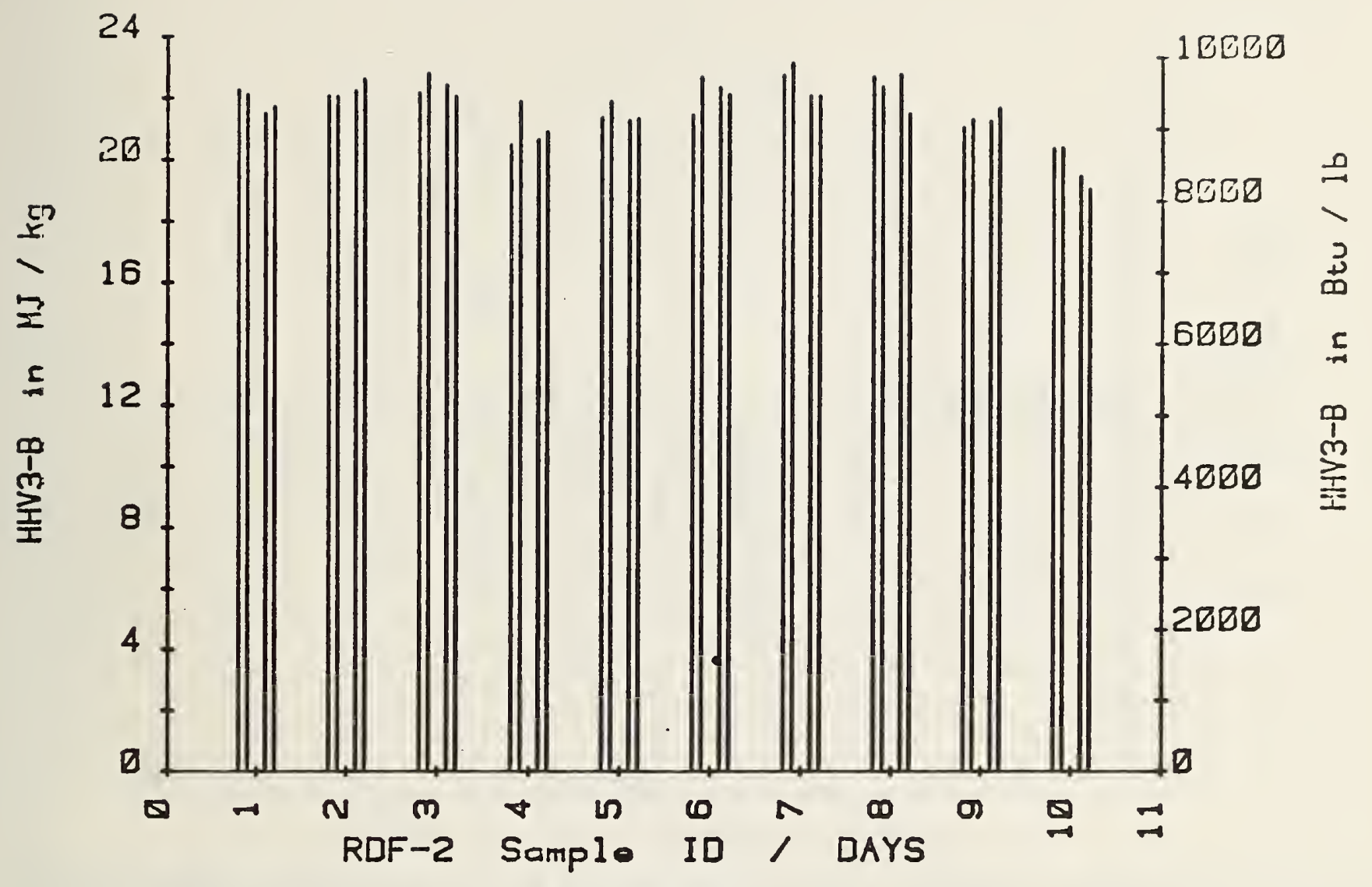

Fig. 12 Variability of HHV3-B

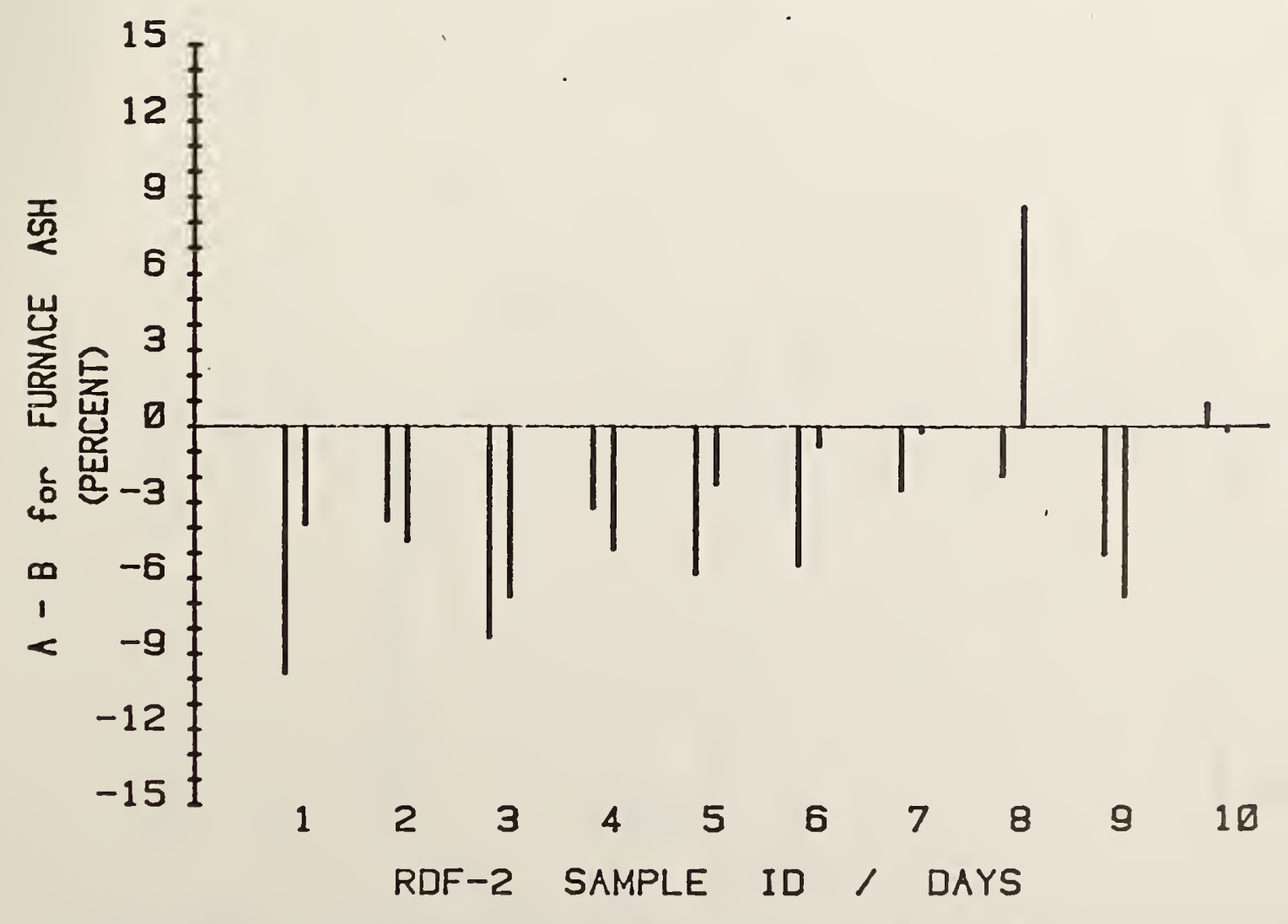

Fig. 13 Effoot of the Sample Split on Furnaco Aoh 


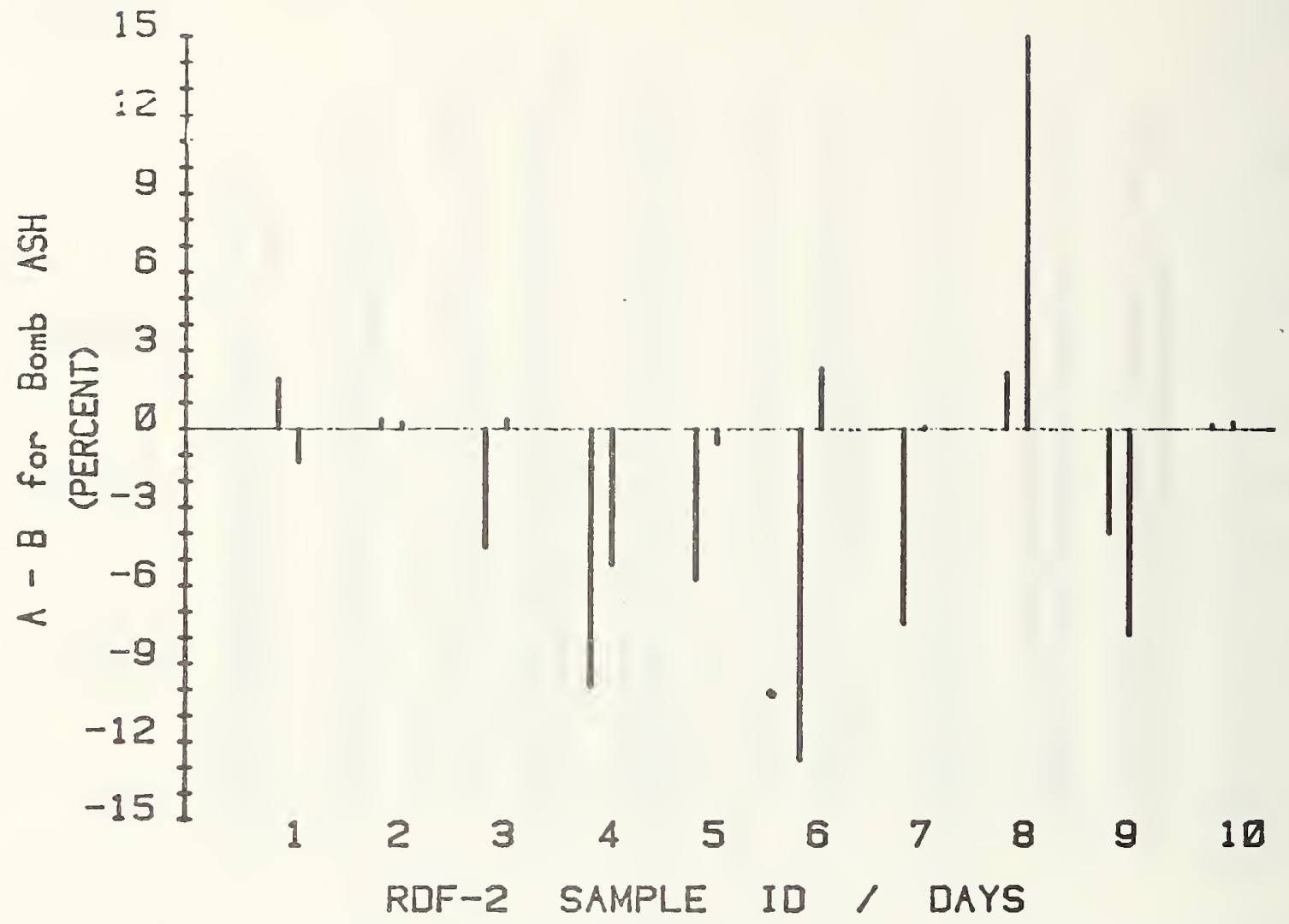

Fig. 14 Effeot of the Sample Split on Bomb Aeh

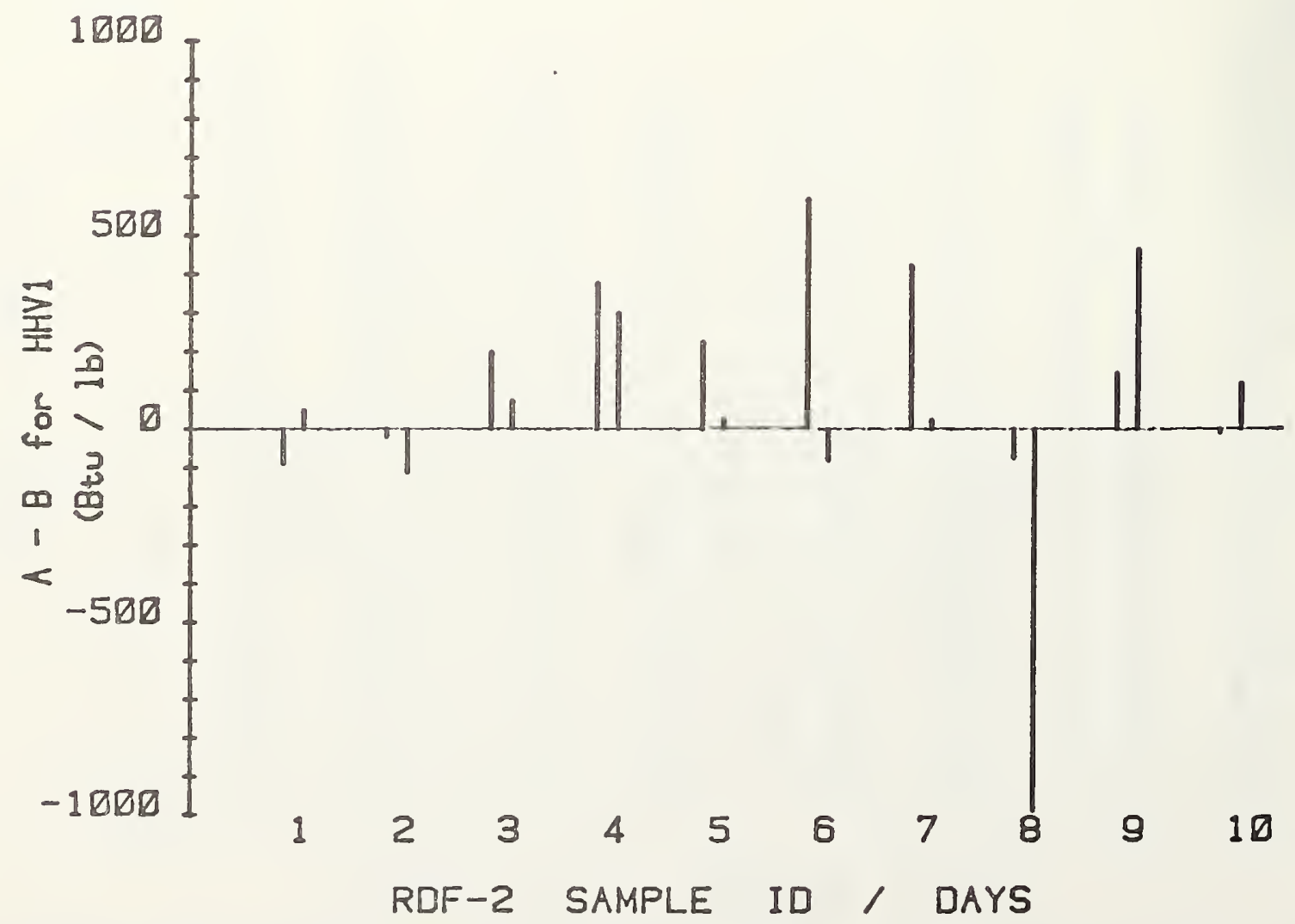

Fig. 15 Effoct of the Sample Split on HHVI 


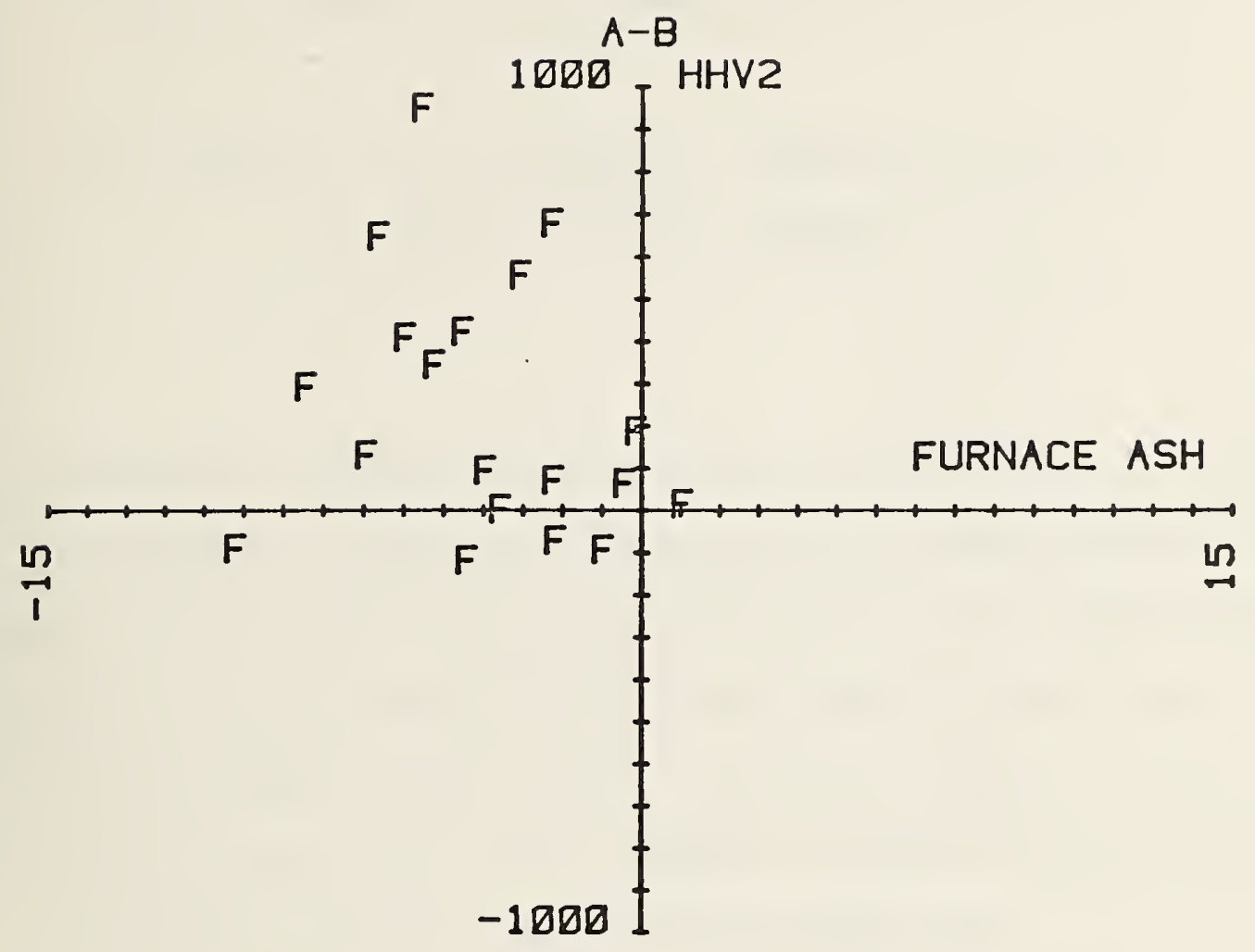

Fig. 16 Corralation of Highor Heating Value and Furnace Aah

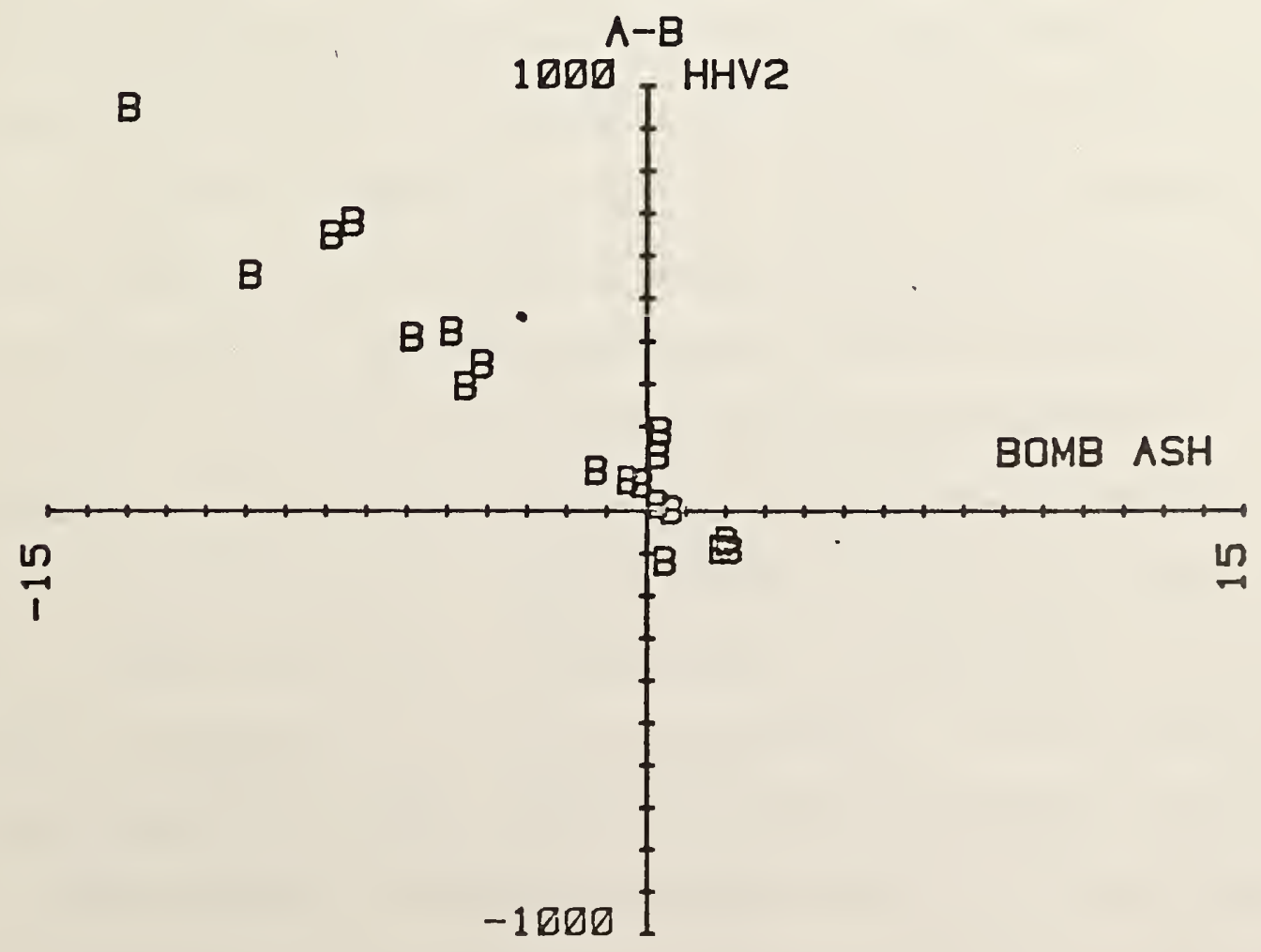

Fig. 17 Corrolation of Highor Hoating Valvo and Bomb Aah 

PART B. 25 GRAM CAPACITY COMBUSTION FLOW CALORIMETER

E.S. Domalski, K.L. Churney, M.L. Reilly, D.R. Kirklin, A.E. Ledford, and D.D. Thornton

\section{Abstract}

A new calorimeter is being developed at the National Bureau of Standards to determine the enthalpies of combustion of kilogram-size samples of municipal solid waste (MSW) in flowing oxygen near atmospheric pressure. The organic fraction of 25 gram pellets of highly processed MSW has been burned in pure oxygen to $\mathrm{CO}_{2}$ and $\mathrm{H}_{2} \mathrm{O}$ in a small prototype flow calorimeter. The carbon content of the ash and the uncertainty in the amount of $\mathrm{CO}$ in the combustion products contribute calorimetric errors of 0.1 percent or less to the enthalpy of combustion.

\section{Introduction}

The ivational Bureau of Standards (NBS) has been mandated by the U.S. Congress through Public Law 94-580, the Resource Conservation and Recovery Act of 1976, to develop guidelines for specifications on recoverable waste materials. NBS has made a commitment to develop a calorimeter to determine the calorific value of kilogram-size samples of minimally processed municipal solid waste (MSW). A large scale calorimeter affords greater credibility because kilogram-size samples of MSW should be more representative of the heterogeneous bulk material than the highly processed gram-size samples currently used in bomb calorimetric determinations.

Combustion of samples in flowing oxygen near atmospheric pressure rather than in the high pressure oxygen of a combustion bomb was adopted for safety considerations. However, the flow technique has not been used in any substantial way to determine the enthalpy of combustion of solids since the 1880's. Its development was discontinued because of the simpler techniques and more quantitative results obtained with the bomb calorimeter. Attaining complete combustion was particularly difficult in a flow system. As a consequence, the first goal of the NBS project was to 
demonstrate that the oxygen flow technique could be used to obtain complete combustion of MSW.

The first step in the program was to build a calorimeter for combustion of 25 gram pellets of highly processed MSW. The sample was burned in the form of pellets to simplify ash collection. The purpose was to estabiish the equivalence of the flow results with those obtained by bomb calorimetry (e.g. with the new NBS 25 gram capacity bomb calorimeter [1]). The preliminary results obtained with the 25 gram flow calorimetric system are described in the following sections.

The early development of the combustor for the 25 gram capacity flow calorimeter dates back to December 1978. An acceptable design and suitable operating procedures for this combustor were established as a result of combustion studies completed in the summer of 1979. Calorimetric measurements were conducted and analyzed between October 1979 and July 1980. The prototype flow calorimeter was used for these measurements while the final calorimeter was being fabricated. All measurements described below were made with the prototype calorimeter. Measurements with the final calorimeter will be started in fiscal year 1981.

Benzoic acid was found to be unsatisfactory as a solid calibrant material for flow work. Pellets melted and burned with a flame that produced large amounts of soot. U1tra-pure carbon powder also proved to be unsatisfactory; it was very difficult to ignite. Flame calorimetry calibrants (gaseous $\mathrm{H}_{2}$ or $\mathrm{CH}_{4}$ ) were not appropriate because an apparatus for quantitative (i.e. $0.01 \%$ ) determination of the amounts of calibrant combusted had not been constructed.

The prototype flow calorimeter was calibrated by burning five pellets of RDF-4 from Lot $A$, whose enthalpy of combustion had been determined previously with a conventional gram-size bomb calorimeter at NBS. The heat of combustion of a different RDF-4, called Lot B, was measured in six experiments. The ash was determined for each experiment in order to verify that the combustible fraction of each lot was the same within experimental uncertainty for both bomb and flow measurements . 
After the RDF-4 experiments, one gram pellets of pure cellulose were test burned in a similar combustor. The pellets ignited easily and burned completely. Subsequently, bomb calorimetric measurements were initiated to establish the enthalpy of combustion of this material so that it can be used as a standard solid calibrant for the flow calorimetric measurements.

The experimental measurements on Lot $A$ and Lot $B$ are described in section two and the calculation of results and an error analysis of the results are given in sections three and four. Results and conclusions are given in section five. 


\section{25 Gram Flow Calorimeter Experimental Work}

\subsection{Apparatus and Samples}

The combustor used in the 25 gram oxygen flow experiments is shown in Fig. 1. The sample pellet (D of Fig. 1) was placed on a quartz plate (F) which had eight radial slots and a small central hole which permitted circulation of oxygen beneath the sample. The plate sat in a quartz crucible (E) which was supported by a nichrome stand $(J)$. Primary oxygen $(H)$ was supplied locally to the sample through a three-port tubular quartz ring which was located immediately above the crucible. The lower edge of the Pyrex thermal shield (C) enclosed the ring and upper edge of the crucible. The outer boundary of the combustor consisted of the stainless steel top (N) and base (I) plus the Pyrex wall (L) which were sealed by rubber gaskets $(B)$. A secondary flow of oxygen $(G)$ swept down between the wall of the combustor and the thermal shield and confined the flow of the products of combustion to the interior of the thermal shield. Gases left the combustor through a stainless steel exit line (A) in the top. The temperatures of the product gases near the exit port of the thermal shield and of the midpoint of the interior of the thermal shield were monitored using Type $K$ thermocouples. The thermocouples were supported in helical Pyrex tubing (K) which stood within the thermal shield.

The combustor was placed in a provisional calorimetric assembly similar to the final design shown in Fig. 2. The provisional assembly consisted of an insulated bell jar filled with water which was circulated by a stirrer ( $J$ of Fig. 2 ) mounted from the bell jar cover. The flow shield (E) caused the water to circulate up past the combustor $(L)$ and down along the inner wall of the jar (analogous to $B$ ). The thin metal disk (K) mounted on the stirrer shaft eliminated leakage of water through the Teflon seal between the stirrer shaft and the bell jar cover (cf. between the shaft and A of Fig. 2). The temperature of the water was measured 


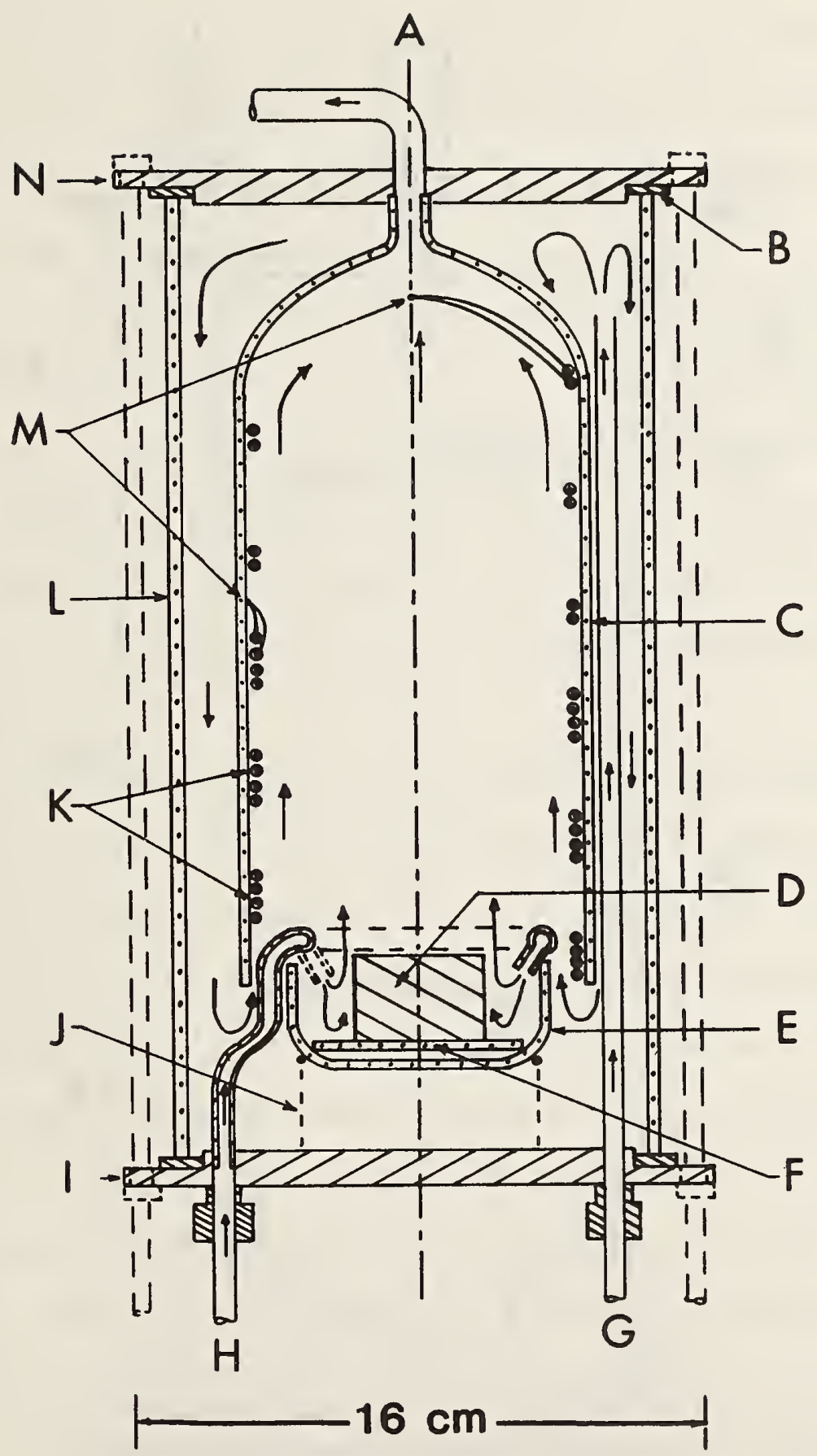

Fig. 1. The 25 Gran Combustor.

Notations: A denotes the product gas exit line, B the gasket, $C$ the thermal shield, D the RDF-4 sample, E the crucible, $F$ the plate, $G$ the secondary oxygen, $H$ the primary oxygen, I the combustor base, $J$ the crucible support, $K$ the thermocouple coils, $L$ the combustor walls, $M$ the thermocouple junctions and $\mathrm{N}$ the combustor Iid. 


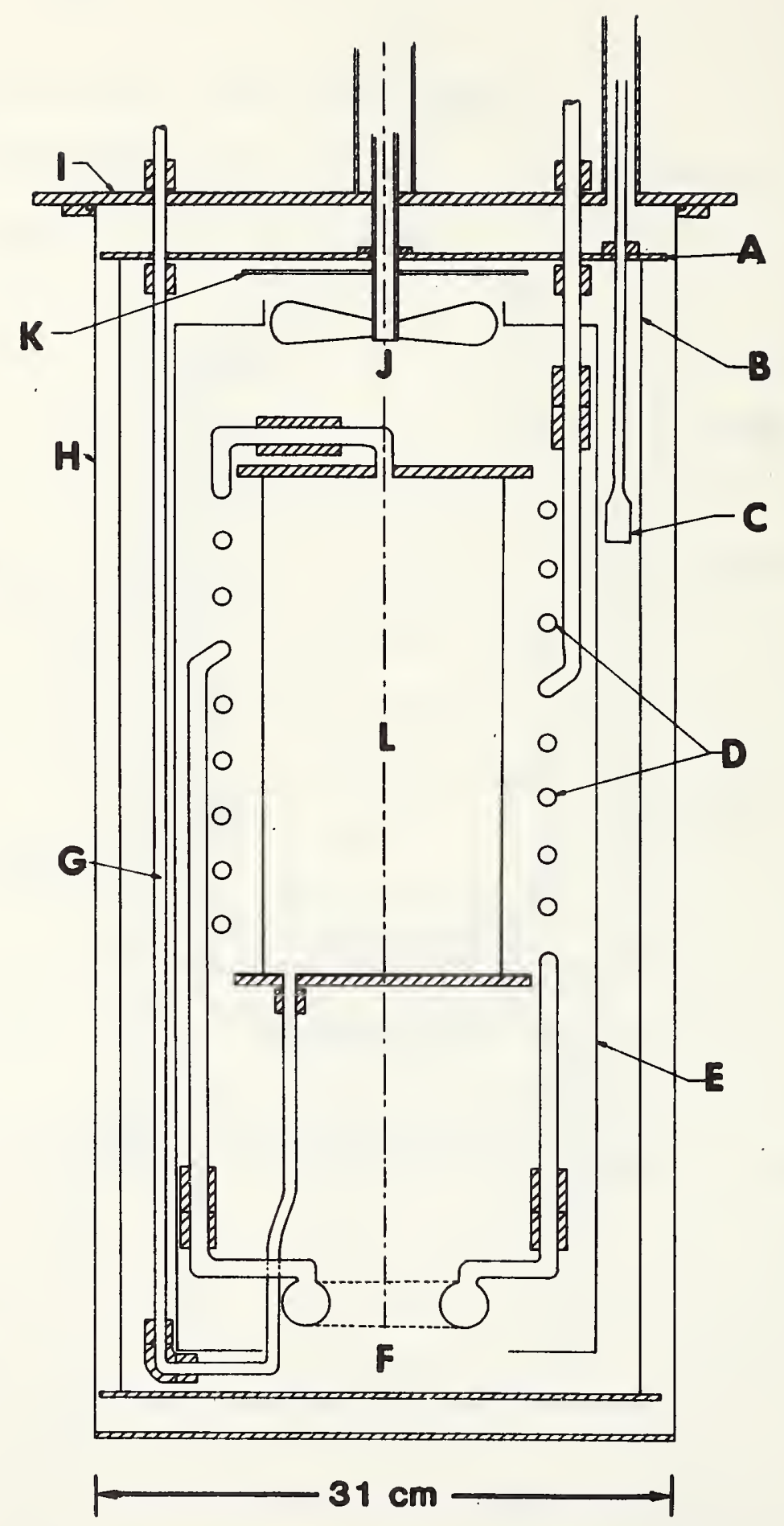

Fig. 2. The 25 Gram Flow Calorimeter.

Notations: A denotes the vessel lid, B the vessel can, C the thermometer, D the exit gas coils, E the flow shield, $F$ the water trap, $G$ the oxygen supply lines, $\mathrm{H}$ the submarine can, I the submarine lid, J the stirrer, $K$ the metal disk and $I$ the combustor. 
with a long stem platinum resistance thermometer $(C)$. The product gases flowed through the helical exit gas coil (D) into the water trap ( $F$ ) and then through a second coil before leaving the calorimeter. The coils and trap were made from Pyrex.

After leaving the calorimeter, the product gases were scrubbed by bubbling through water and then were diluted with dry gas before the entire gas stream was passed successively through a variable orifice flowmeter and non-dispersive infrared detectors for measuring the concentrations of $\mathrm{CO}$ and $\mathrm{CO}_{2}$. Analysis of the product gases in the combustor development experiments by both mass spectrometry and conventional infrared spectroscopy showed $\mathrm{CO}$ to be the only species present due to incomplete combustion.

Ordinarily, the oxygen supplied to the calorimeter was saturated with water vapor by bubbling the gas through two gas washing flasks connected in series.

Type $\mathrm{K}$ thermocouples were used to measure the temperature of the oxygen as it entered the calorimeter and the temperature of the product gases both as they left the calorimeter and at the $\mathrm{CO}$ and $\mathrm{CO}_{2}$ detectors. The pressure of the product gases at the $\mathrm{CO}$ and $\mathrm{CO}_{2}$, detectors was monitored with a capacitance digital manometer. The analog output of the $\mathrm{CO}, \mathrm{CO}_{2}$, and pressure detectors and all thermocouple voltages were monitored using a $51 / 2$ digit voltmeter ( $1 \mu V$ sensitivity), a ten channel scanner, and a 16-bit minicomputer. Calorimeter water temperatures were measured manually by determining the resistance of the platinum resistance thermometer with a Mueller bridge and an electronic null detector.

Sample pellets for the 25 gram experiments were prepared from a blended powder of minus $0.5 \mathrm{~mm}$ particle size. The powder was made by milling large batches of minus $2 \mathrm{~cm}$ MSW from which most of the metals, glass and entrained inorganics had been removed. This powder is referred to as RDF-4. The cylindrical pellets, which were $3.5 \mathrm{~cm}$ in diameter and a $2.5 \mathrm{~cm}$ high, were prepared by pressing the powder in a die using a force ranging from 45 to $160 \mathrm{kN}(10,000$ to $36,0001 \mathrm{bs})$. The residual moisture content of the powder was determined to be about $5 \%$ using the 
ASTM test E790, test for residual moisture in a refuse-derived fuel analysis sample.

\subsection{Description of a Typical Experiment}

In a typical experiment, the calorimeter was assembled and the combustor was flushed with pure oxygen. The primary and secondary oxygen flow rates were set at approximately 8 and 5 liters per minute $\left(L \cdot \mathrm{m}^{-1}\right)$, respectively. The temperature of the calorimeter water was recorded as a function of time for approximately twenty minutes after a steady drift rate was attained. The sample was ignited by passing electrical current through an iron fuse wire (not shown in Fig 1) which was in contact with the top of the pellet. A 25 gram pellet typically burned within fourteen minutes. Upon ignition the pellet burned with a diffusion flame which spread over its entire surface within the first 30 seconds. The flame temperature was determined to be greater than $1500{ }^{\circ} \mathrm{C}$. A small but detectable peak in the Co concentration occurred at about 3 minutes. The surface flame disappeared at about the eighth minute. The glowing sample continued to burn internally leaving a porous ash structure that had almost the same shape as the original pellet. The product gases contained increasing amounts of $\mathrm{C} 0$, which reached a peak near the eleventh minute. However, the ratio of $\mathrm{CO}$ to $\mathrm{CO}_{2}$ remained low. A typical $\mathrm{CO}-\mathrm{CO}_{2}$ composition profile of the product gases is shown in Fig 3.

The temperature of the product gas stream at the top of the combustor rose quickly to a peak near $600{ }^{\circ} \mathrm{C}$ some 30 seconds after ignition and then decayed exponentially as the combustion proceeded. The product gases were cooled in the exit gas coils and approximately $85 \%$ of the water formed was collected in the trap. The gas was further cooled in the second exit coil. Throughout the combustion, the temperature of the product gases leaving the calorimeter was less than $0.1{ }^{\circ} \mathrm{C}$ above the temperature of the calorimeter water.

During the first seven minutes the temperature of the calorimeter water rose linearly at a rate near $0.25{ }^{\circ} \mathrm{C} / \mathrm{min}$. Subsequently, the temperature of the calorimeter water continued to rise at a progessively diminishing rate until a final 


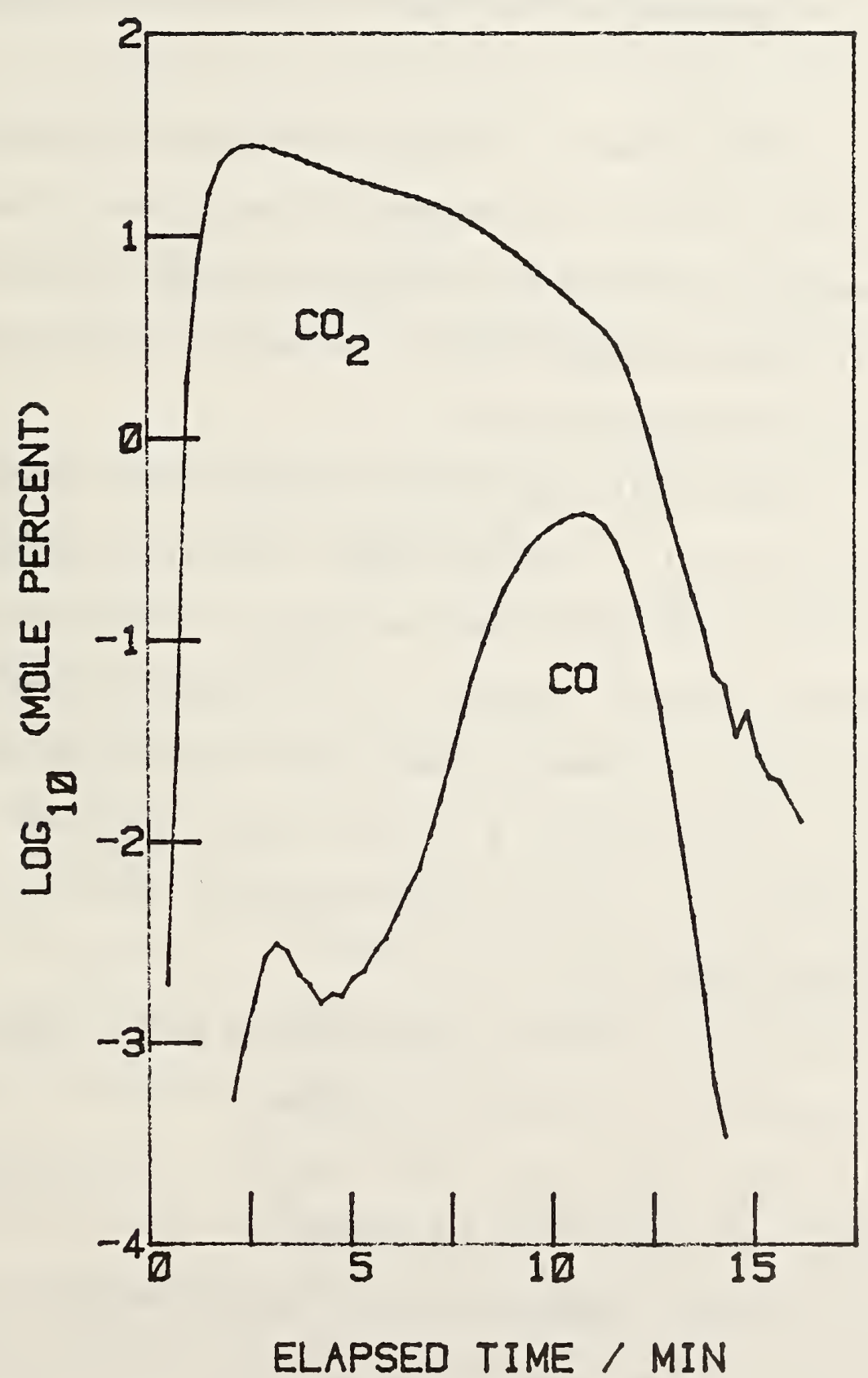

Fig. 3. The $\mathrm{CO}-\mathrm{CO}_{2}$ Composition of the Product Gases vs the Elapsed Time from Ignition for a Typical Experiment. 
steady-state drift rate was attained about forty to forty-five minutes after ignition. To establish the final drift rate, temperatures were recorded for at least thirty additional minutes. The temperature rise (from the end of the initial drift period to the beginning of the final drift period) was typically $2.3{ }^{\circ} \mathrm{C}$.

After disassembly of the calorimeter, the ash and the sample crucible were weighed. The carbon content of the ash was determined from the sum of the mass decrease produced by heating the ash and crucible with an oxygen-gas flame plus subsequent analysis for the remaining total occluded carbon. The carbon in the ash ranged from 0.015 to $0.1 \%$ of the initial sample mass.

A small amount $(<1 \mathrm{mg})$ of white residue was deposited on the thermal shield during each run. In about one-half of the experiments, the water condensed in the trap (see Fig. 2) was greenish blue rather than clear. The trap also contained a black residue. Apparently this was trapped fly ash which had partially dissolved. Analysis showed that no carbon was present in the residue and that the colored solution contained $\mathrm{Fe}, \mathrm{Cr}, \mathrm{Ni}, \mathrm{Na}$ and $\mathrm{K}$ (i.e. in the range of $100 \mu \mathrm{g} \cdot \mathrm{ml}^{-1}$ ) in addition to the usual acidity ( 0.1 to 0.2 milliequivalents per milliliter).

\section{Calculation of 25 Gram Flow Results}

Results are calculated using a format adapted from the work of Prosen et al. [2] which treats only the case where all reactants are gaseous.

$$
E \Delta T / m=-\left(\Delta H_{s t}+\Delta H_{i c}+\Delta H_{g}+\Delta H_{v}+\Delta H_{i g n}\right)
$$

In eq. $1, E$ is the energy equivalent of the calorimeter,

$\Delta T$ is the corrected temperature rise of the calorimeter,

$m$ is the mass of the pelleted RDF-4 sample,

$\Delta \mathrm{H}_{\text {st }}$ is the enthalpy change of an assumed standard flow reaction for one gram of RDF-4 in which all combustion products are at the same reference temperature $T_{h}$. 
The remaining four enthalpy change terms (per gram of RDF-4) on the right side of eq. 1 account for the conditions of a particular experiment. They are as follows:

$\Delta \mathrm{H}_{i \mathrm{c}}$ is the correction for the incomplete combustion of all $\mathrm{CO}$ to $\mathrm{CO}_{2}$, $\Delta H_{g}$ is the correction for the net heat transport by oxygen entering and product gases leaving the calorimeter at some temperature other than $T_{h}$,

$\Delta H_{v}$ is the correction for the heat of vaporization of water formed in the combustion that is lost from the calorimeter with the product gases,

and

$\Delta \mathrm{H}_{i g n}$ is the correction for the enthalpy of combustion of the iron

fuse wire used to ignite the RDF-4 sample.

The electrical work done to heat the fuse wire in order to ignite the sample has been assumed to be negligible.

An energy equivalent of the calorimeter was calculated for each of the experiments involving Lot $A$ using a value of $\Delta \mathrm{H}_{\text {st }}$ derived from bomb calorimetric measurements. The value of $\Delta H_{s t}$ for Lot $B$ was calculated using the average $E$ from the Lot $A$ experiments. The method used to calculate $\Delta T$ is given in section 3.1 and the enthalpy terms on the right side of eq. 1 are given in section 3.2 to 3.7. Results are tabulated in section 3.8.

\subsection{Calculation of $\Delta T$}

The value for $\Delta T$ is calculated in the same manner as in bomb calorimetry. The temperature of the calorimeter water was measured with the platinum thermometer immersed in the stirred water outside of the flow shield. As the resistance, R, of the thermometer varies nearly linearly with $T$ over the range of temperature $\left(<4{ }^{\circ} \mathrm{C}\right)$ involved in calculating $\Delta T$, the corrected resistance rise, $\Delta R$, was calculated in place of $\Delta T$, using eq. 2. The only effect is to change the units of $E$ to $\mathrm{J} \cdot \Omega^{-1}$ (joules per ohm); $1 \Omega$ is approximately $10{ }^{\circ} \mathrm{C}$. 


$$
\Delta R=R_{f}-R_{j}-k \int_{t_{j}}^{t_{f}}\left(R_{\infty}-R\right) d t
$$

In eq. $2, R_{f}$ and $R_{i}$ are resistances at the end, time $t_{f}$, and the beginning, time $t_{j}$, of the main period. The symbols $k$ and $R_{\infty}$ are called the cooling constant and the convergence resistance, respectively. The values of $k$ and $R_{\infty}$ are calculated for each experiment by simultaneously fitting eq. 3 to both the initial and final drift data using a computer program written by R.L. Nuttall [3].

$$
d R / d t=k\left(R_{\infty}-R\right)
$$

The results are summarized in table 1. Experiment numbers in column 1 indicate the order in which they were performed followed by a letter indicating the lot designation.

Oxygen was supplied to the calorimeter at a constant flow rate throughout each experiment. In experiments 1 through 9, the inlet oxygen was saturated with water vapor. The assumption was made that $k$ and $R_{\infty}$ were the same as if no oxygen was flowing during either drift period since this is, strictly speaking, required for the validity of eqs. 1 through 3. The length of the main period was taken to be 41 minutes or longer, except for experiment 5B where insufficient final drift data were available. Main periods of shorter duration yielded values of $k$ smaller than those in table 1 which indicate eq. 3 does not apply until $t_{f}-t_{j} \geq 41$ minutes.

The oxygen supplied to the calorimeter in experiments 10 and 11 was dry. Values of $k, R_{\infty}$ and $\Delta R$ in experiments 10 and 11 were adjusted to the average $k$ of the first nine experiments. It was assumed that the observed final drift rates in experiments 10 and 11 were too small due to vaporization of water from the calorimeter in the final drift period. The correction, $\delta \Delta R$, added to $\Delta R$ was:

$$
\delta \Delta R=-\left(k-k_{c}\right)\left[\left(R_{\infty}-R_{j}\right)\left(t_{f}-t_{j}\right)-\int_{t_{j}}^{t_{f}}\left(R_{\infty}-R\right) d t\right]
$$


บ

总

㐫

อ

总

$\stackrel{5}{\infty}$

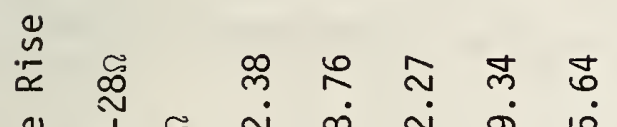

₹

$\stackrel{\substack{E \\ \frac{\pi}{2}}}{\frac{\pi}{2}}$

๓

I

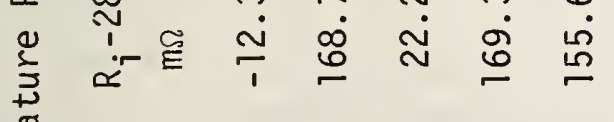

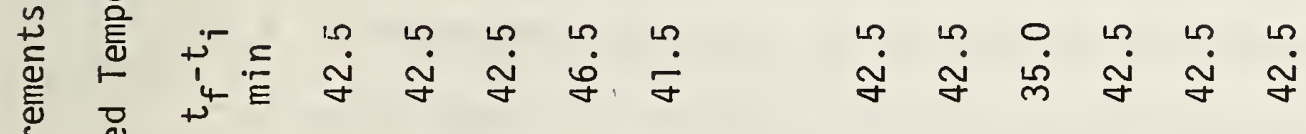

$\stackrel{0}{ \pm}$

ธ

芩

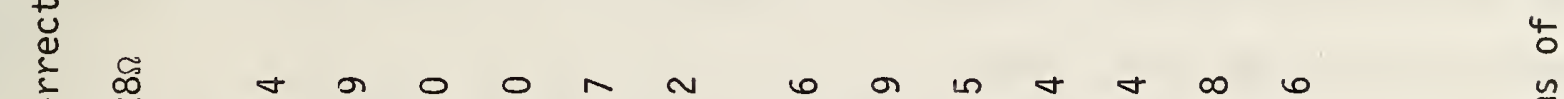

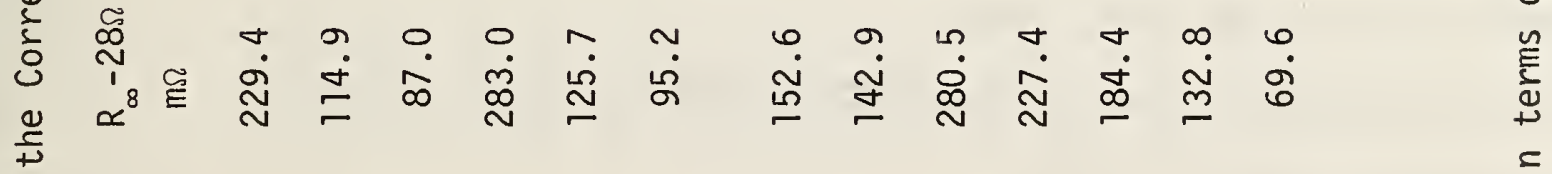

$4+$

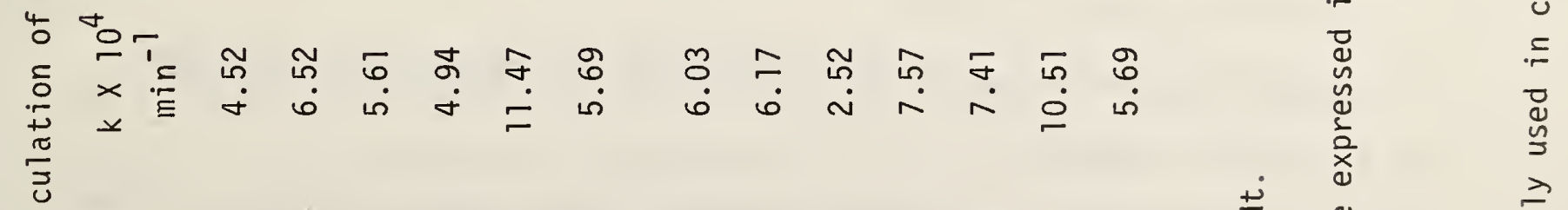

岕

$\stackrel{\square}{ \pm}$

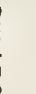

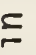

赵

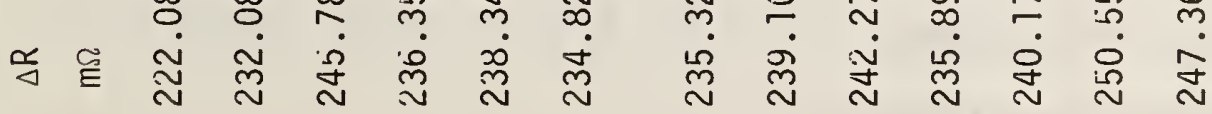

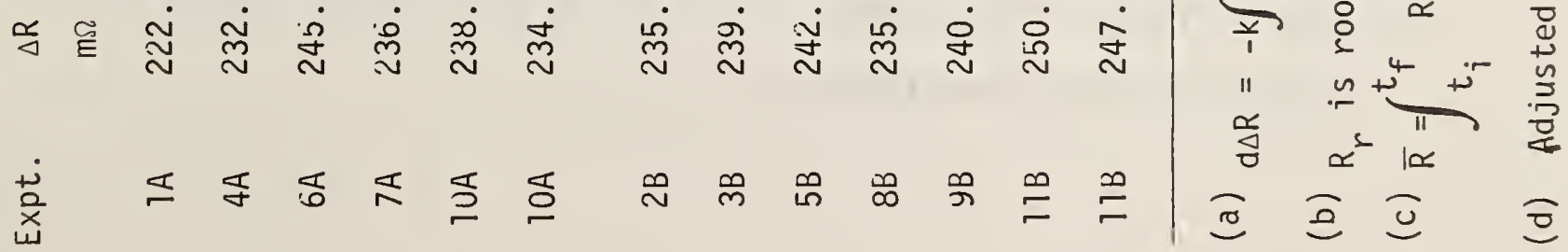


In eq. $4, k_{c}$ is the average cooling constant of the first nine experiments, and was calculated to be $5.69 \times 10^{-4} \min ^{-1}$. Adjusted values are listed in the second row of results for experiments 10 and 11 .

\subsection{Calculation of Enthalpy Changes}

The changes in state corresponding to each of the enthalpy changes in eq. 1 are given in succeeding sections. The calculations and the changes in state are based on the following assumptions:

a) The pressures of the gases entering and leaving the calorimeter are constant and differ negligibly from one atmosphere (101.325 kPa); the pressure in the combustor at times $t_{i}$ and $t_{f}$ is one atmosphere. We have assumed that the gases (or gas mixtures) obey the perfect gas law.

b) Oxygen entering the calorimeter is pure except for the presence of water vapor and the moles of oxygen within the boundary of the calorimeter are the same at times $t_{j}$ and $t_{f}$.

c) In the combustion reaction, the nitrogen, sulfur, and chlorine of the RDF-4 sample that are not retained in the ash are completely converted to $N_{2}(g)$, $\mathrm{SO}_{2}(\mathrm{~g})$, and aqueous $\mathrm{HCl}$, respectively. The last species is assumed to be condensed completely into the water trap.

d) The only product due to incomplete combustion of the organic fraction of RDF-4 is CO.

e) The energy equivalent of the calorimeter is assumed to be the same at times $t_{j}$ and $t_{f}$.

f) The temperature, $T_{h}$, of the isothermal flow process (analogous to the isothermal bomb process of bomb calorimetry) is equal to $T_{j}$, the temperature of the calorimeter at time $t_{j}$.

g) The correction for viscous effects and kinetic energy losses of the flowing gases is negligible. 


\subsection{Calculation of $\Delta H_{\text {st }}$ from Bomb Calorimetry}

The change in state corresponding to $\Delta H_{s t}$ for the flow experiments is given by the equation for the standard flow reaction for RDF-4,

$$
\begin{aligned}
& {\left[\left(n_{1}+n_{2}\right) O_{2}+n_{3} H_{2} \mathrm{O}\right]\left(g, T_{i}\right)+1 \text { gram RDF-4 }\left(s, T_{i}\right)=} \\
& {\left[n_{5} \mathrm{CO}_{2}+n_{6} N_{2}+n_{8} \mathrm{SO}_{2}+n_{2} \mathrm{O}_{2}\right]\left(g, T_{i}\right)+m^{\prime} \operatorname{gram} \text { Ash }\left(s, T_{i}\right)+} \\
& {\left[n_{9} \mathrm{HCl}+n_{10} \mathrm{H}_{2} \mathrm{O}\right]\left(\operatorname{soln}, T_{i}\right) ; P=1 \mathrm{~atm} .}
\end{aligned}
$$

In eq. $5,(g)$ refers to gas, (s) to solid, and (soln) to solution; $n_{1}$ is the number of moles of $\mathrm{O}_{2}$ required to convert one gram of RDF-4 having some residual moisture to $n_{5}$ moles of $\mathrm{CO}_{2}(\mathrm{~g}), \mathrm{n}_{6}$ moles of $\mathrm{N}_{2}(\mathrm{~g}), \mathrm{n}_{8}$ moles of $\mathrm{SO}_{2}(\mathrm{~g}), \mathrm{n}_{9}$ moles aqueous $\mathrm{HCl}$, and $n_{10}$ moles of liquid water. The quantities $n_{2}$ and $n_{3}$ are the moles of oxygen in excess of stoichiometry and water vapor that enter the calorimeter during the main period, respectively. $P=1 \mathrm{~atm}$ indicates the total pressure of products or reactants is one atmosphere.

The molar quantities $n_{7} n_{5}, n_{6}, n_{8}, n_{9}$ and $n_{10}$ are calculated from the elemental composition determined at the time of the bomb calorimetric measurements. The quantity $n_{10}$ is the most important. In the shortcut procedure of this work, $n_{10}$ minus the moles of water per gram RDF-4 remaining in the water trap is used to evaluate the largest of the correction term on the right side of eq. $1, \Delta H_{v}$. The values of $n_{10}$ were checked in the last two flow experiments, as discussed in section 3.6 .

The elemental composition determinations and the energy of combustion data determined by bomb calorimetry are summarized in table 2. Results are listed for moisture-free RDF-4; composition is given in terms of mass fraction. Each lot was homogenized in a vee-blender, coned, and quartered. An elemental composition determination, two moisture determinations, and a single measurement of the energy of combustion were made using samples drawn from each quarter. The mass fraction of $H$ for Lot $A$ actually used in the computations is listed in parentheses and is discussed in section 3.6 . 
Table 2 Bomb Calorimetric Measurements on RDF-4 Samples

Elemental Composition (mass fraction, moisture free)

\begin{tabular}{|c|c|c|c|c|c|c|c|}
\hline Quarter & C & $H$ & N & 0 & $S$ & $\mathrm{Cl}$ & Ash \\
\hline & & & Lot $A$ & & & & \\
\hline 1 & .4273 & .0637 & .0079 & .2653 & .0047 & .0027 & .2283 \\
\hline 2 & .4386 & .0645 & .0083 & .2601 & .0048 & .0029 & .2207 \\
\hline 3 & .4259 & .0634 & .0077 & .2696 & .0048 & .0027 & .2259 \\
\hline 4 & .4367 & .0629 & .0074 & .2596 & .0049 & .0030 & .2256 \\
\hline AVERAGE & .4321 & .0636 & .0078 & .2637 & .0048 & .0028 & .2252 \\
\hline & & $(.0523)$ & & & & & \\
\hline & & & Lot $B$ & & & & \\
\hline 1 & .4249 & .0546 & .0056 & .2546 & .0014 & .0035 & .2554 \\
\hline 2 & .3925 & .0511 & .0049 & .3048 & .0013 & .0034 & .2420 \\
\hline 3 & .3576 & .0462 & .0046 & .3529 & .0013 & .0034 & .2340 \\
\hline 4 & .4174 & .0545 & .0044 & .2822 & .0013 & .0043 & .2353 \\
\hline AVERAGE & .3981 & .0516 & .0049 & .2986 & .0013 & .0036 & .2418 \\
\hline
\end{tabular}

(a) Value actually used, see section 3.6. 
Table 2 (continued)

Energy of Combustion (moisture free)

Quarter

1

2

3

4

AVERAGE

Std. Dev. Mean

Imprecision (b)
Lot A

HHV2 ${ }^{(a)}$

$3 \cdot g^{-1}$
17010

17087

16889

17054

17010

$\pm 43(.25 \%)$

$\pm 137(.80 \%)$

Lot B

HHV2

$\begin{array}{cc}J \cdot g^{-1} & \text { Btu } \cdot 1 b^{-1} \\ 17208 & 7398\end{array}$

17291

7434

7261

7331

7331

$\pm 18$

$+24(.14 \%) \quad+10$

$\pm 70$

(a) HHV2: Energy of Combustion, moisture free; $1 \mathrm{~kJ} \cdot \mathrm{g}^{-1}=429.9226 \mathrm{Btu} \cdot 1 \mathrm{~b}^{-1}$.

(b) Calculated as 3.18 times the std. dev. of mean. 
To calculate the parameters in eq. 5 for the flow experiments, the following assumptions were made:

a) The four fractions of each lot are identical.

b) The ash in the bomb and flow reactions are identical

(i.e. in mass fraction and chemical composition).

The results are summarized in table 3 for RDF-4 samples which have the appropriate residual moisture contents determined at the time of the flow measurements: $5.08 \pm 0.01$ and $3.96 \pm .01$ mass percent for Lot $A$ and Lot $B$, respectively.

To calculate $\Delta H_{s t}$ from HHV2 of the bomb measurements, the following additional assumptions were made:

a) The net correction to obtain HHV2 for the bomb reaction at atmospheric pressure from the measured HHV2 at 30 atmosphere pressure of oxygen is negligible.

b) The change in HHV2 or $\Delta H_{s t}$ for the flow reaction with temperature can be neglected.

The calculation of $\Delta \mathrm{H}_{\mathrm{st}}$ for both lots is summarized in table 4 . HHV2 is the average of the four fractions for each lot; the uncertainty is the imprecision corresponding to $95 \%$ confidence limit and three degrees of freedom. HHV2-HHV(AD) is the correction to convert one gram of dry RDF-4 to one gram of RDF-4 having the moisture contents appropriate to the flow experiments. The parameter $\Delta \mathrm{nRT}$ is the pressure-volume correction to obtain $\Delta H_{s t}$ from HHV2. $\Delta n$ is the sum of moles of $\mathrm{CO}_{2}(\mathrm{~g}), \mathrm{N}_{2}(\mathrm{~g})$, and $\mathrm{SO}_{2}(\mathrm{~g})$ minus the moles of stoichiometric $\mathrm{O}_{2}$. $\Delta \mathrm{n}$ is given in table 3.

\subsection{Calculation of $\Delta H_{i c}$}

$\Delta H_{j c}$ was calculated for the change in state

$$
n_{4} \mathrm{CO}_{2}(g)=n_{4} \mathrm{CO}(g)+\left(n_{4} / 2\right) O_{2}(g) ; T_{j} ; P=1 \mathrm{~atm}
$$


Table 3. Standard Flow Reaction

Composition of one gram of RDF-4

formula: $\left[\begin{array}{llllllll}\mathrm{C}_{\mathrm{a}} & \mathrm{H}_{b} & \mathrm{~N}_{\mathrm{C}} & \mathrm{O}_{\mathrm{d}} & \mathrm{S}_{\mathrm{e}} & \mathrm{Cl}_{\mathrm{f}}\end{array} \mathrm{(H}_{2} \mathrm{O}\right)_{\mathrm{h}}\left(\mathrm{m}^{\prime}\right.$ grams Ash)

Symbol mmol, Lot $A$

34.15

a

b

c

d

e

$f$

h

$m^{\prime}$ $59.9(49.6)^{(a)}$

0.53

15.64

0.15

0.075

2.82

0.2138 mmol, Lot $B$

31.83

4.92

0.34

17.92

0.039

0.10

2.20

0.2322

Values of Quantities in the Standard Flow Reaction, eq. 5.

\begin{tabular}{|c|c|c|c|}
\hline Symbol ${ }^{(b)}$ & Species & $\mathrm{mmol}$, Lot $A$ & mmol, Lot B \\
\hline$n_{1}$ & $\mathrm{O}_{2}(\mathrm{~g})$ & $41.44(38.88)^{(a)}$ & 35.18 \\
\hline$n_{5}$ & $\mathrm{CO}_{2}(\mathrm{~g})$ & 34.15 & 31.83 \\
\hline$n_{6}$ & $\mathrm{~N}_{2}(\mathrm{~g})$ & 0.27 & 0.17 \\
\hline$n_{8}$ & $\mathrm{SO}_{2}(\mathrm{~g})$ & 0.15 & 0.039 \\
\hline$n_{9}$ & $\mathrm{HCL}$ (aq) & 0.075 & 0.10 \\
\hline$n_{10}$ & $\mathrm{H}_{2} \mathrm{O} \quad(\mathrm{aq})$ & $32.73(26.6)^{(a)}$ & 26.75 \\
\hline$\Delta n^{(c)}(s$ & text) & $-6.87(-4.29)^{(a)}$ & -3.14 \\
\hline $\mathrm{m}^{\prime \prime}$ & Ash & 0.2138 & 0.2322 \\
\hline
\end{tabular}
(a) Value actually used, see section 3.6.
(b) $n_{1}=a+e+b / 4-f / 4-d / 2, n_{5}=a, n_{6}=c / 2, n_{8}=e, n_{g}=f, n_{10}=b / 2+h-f / 2$
$\Delta n=n_{5}+n_{6}+n_{8}-n_{1}$ 


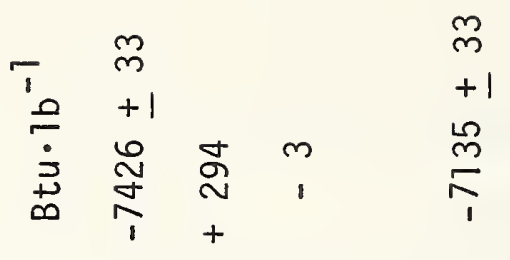

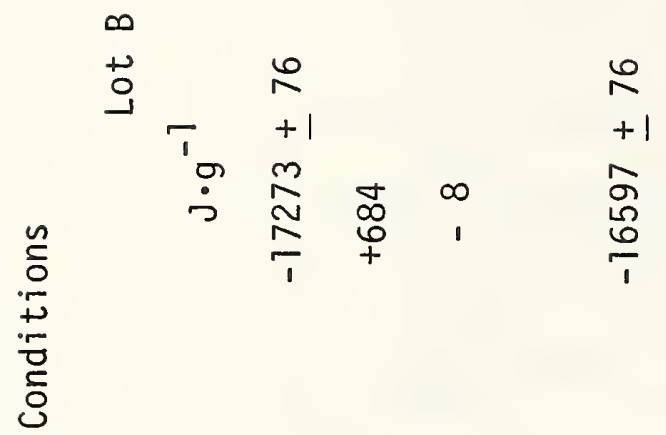

총

는

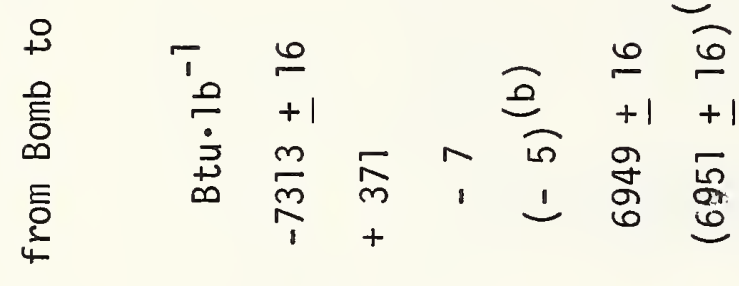

营

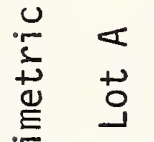

0
0
0
4
0
5
0
5
$\frac{1}{0}$
0
0
0

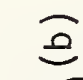

2

๘ิ

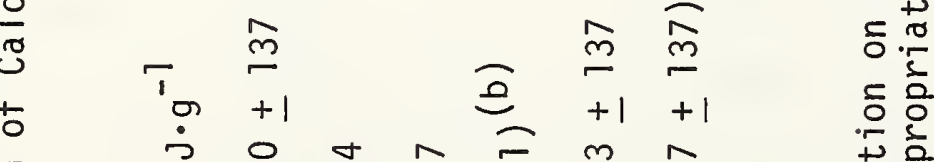

등

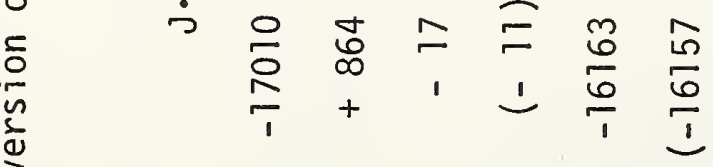

느응

농유. 음

害步 过

4 告 \&

을

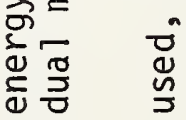

$\frac{0}{2}$

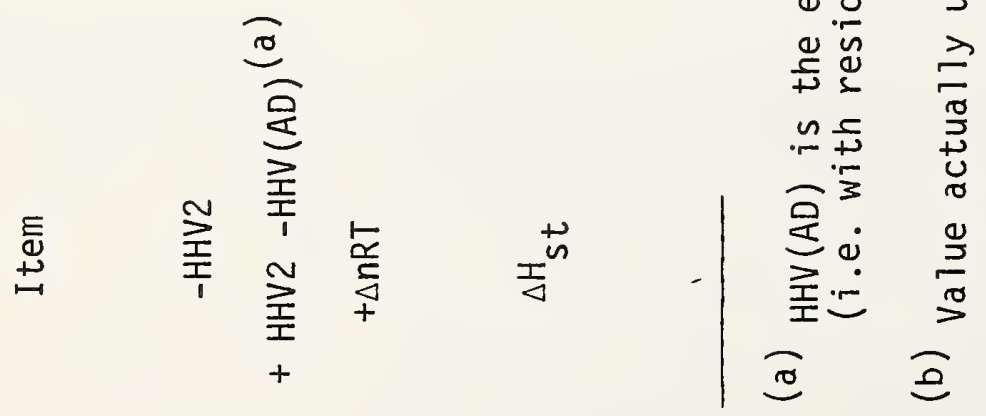


where $n_{4}$ is the number of moles of $C 0$ per gram RDF-4. The enthalpy change per mole of CO was taken as $298 \mathrm{~kJ} \cdot \mathrm{mol}^{-1}$ from reference 4. The value of $n_{4}$ was calculated using the measured volume flow rate of the gases entering the co detector and the pressure and temperature of the gases leaving the detector. During a combustion run, the latter parameters were constant to $2 \%$ for the flow rate, 0.2 to $0.5 \mathrm{kPa}$ for the pressure, and $0.2{ }^{\circ} \mathrm{C}$ for the temperature. The $\mathrm{C} 0$ detector was calibrated using known concentrations of $0.1,0.3,0.6,1$, and $2 \mathrm{~mol} \% \mathrm{co}$ in nitrogen.

\subsection{Calculation of $\Delta \mathrm{H}_{\mathrm{g}}$}

The reaction corresponding to $\Delta H_{g}$ is

$$
[X]\left(g, T_{i n}\right)+[Y]\left(g, T_{j}\right)=[X]\left(g, T_{j}\right)+[Y]\left(g, T_{e x}\right)
$$

where

$$
x=\left(n_{1}+n_{2}-n_{4} / 2\right) 0_{2}+n_{3} H_{2} 0
$$

and

$$
Y=\left(n_{5}-n_{4}\right) C O_{2}+n_{4} \mathrm{CO}+n_{6} N_{2}+n_{8} S_{2}+n_{2} O_{2}+\left(n_{3}+n_{7}\right) H_{2} O
$$

In eq. $7, T_{i n}$ and $T_{e x}$ are the temperatures of the gases entering (in) and leaving (ex) the calorimeter, respectively. $T_{j}$ corresponds to $T_{h} \cdot n_{7}$ is the moles of water vapor leaving the calorimeter in excess of the amount, $n_{3}$, entering during the main period. $\Delta H_{g}$ was calculated using eq. 8.

$$
\begin{aligned}
& \Delta H_{g}=\Delta H_{g 1}+\Delta H_{g 2}+\Delta H_{g 3} ; \\
& \Delta H_{g 1} \cong\left(n_{2} C_{2}+n_{3} C_{3}\right)\left(T_{e x}-T_{i n}\right) ; \\
& \Delta H_{g 2} \cong n_{1} C_{2}\left(T_{j}-T_{i n}\right) ; \\
& \Delta H_{g 3} \cong\left(n_{7} C_{3}+n_{5} C_{5}\right)\left(T_{e x}-T_{j}\right) .
\end{aligned}
$$

$C_{2}, C_{3}$, and $C_{5}$ are the molar heat capacities of gaseous oxygen, water, and $\mathrm{CO}_{2}$ and were taken as the values given in reference 4 at $25^{\circ} \mathrm{C}$. The approximate equalities assume $n_{4}$ and $n_{6}$ are negligible. $T_{\text {in }}$ and $T_{\text {ex }}$ were assumed to equal 
respectively, the room temperature, $T_{r}$, and $T$, the temperature corresponding to the average resistance, $R$, of the platinum thermometer over the main period listed in the last column of table 1 . The numerical value of $\Delta \mathrm{H}_{\mathrm{gl}}$ is about $93 \%$ of $\Delta H_{e}$ in our experiments. Thus $\Delta H_{g}$ is quite insensitive to the value selected for $T_{h}$ (i.e. here $T_{j}$ ).

The values for $n_{1}$ and $n_{5}$ were taken from table 3 . The value of $n_{2}$ was calculated from $n_{1}$ and $\left(n_{1}+n_{2}\right)$. The value of $\left(n_{1}+n_{2}\right)$ was calculated from the sum of the measured volume flow rates of the primary and secondary oxygen. $n_{3}$ was calculated assuming the inlet oxygen in experiments 1 through 9 was saturated with water vapor at room temperature. Values of $n_{7}$ were calculated as discussed in section 3.6 and are given in table 6. Temperature differences were calculated using the last three columns of table 1 and the conversion factor of $9.903 \Omega$ (ohms) difference equals $1 \mathrm{~K}$ difference in temperature.

\subsection{Calculation of $\Delta H_{V}$}

The change in state corresponding to $\Delta \mathrm{H}_{\mathrm{v}}$ is

$$
\begin{aligned}
& Z\left(\operatorname{soln}, T_{j}\right)+\left(n_{7}+n_{3}^{i}-n_{3}^{f}\right) H_{2} O\left(g, T_{j}\right)= \\
& {\left[Z+\left(n_{7}+n_{3}^{i}-n_{3}^{f}\right) H_{2} O\right]\left(\operatorname{soln}, T_{j}\right) ; P=1 \mathrm{~atm} .}
\end{aligned}
$$

where $Z$ is defined as

$$
\mathrm{Z}=\mathrm{n}_{8} \mathrm{H}_{2} \mathrm{SO}_{4}+\mathrm{n}_{9} \mathrm{HCl}+\mathrm{n}_{10} \mathrm{H}_{2} \mathrm{O} \text {. }
$$

In eq. $9, n_{7}$ is the net moles of water vaporized from the calorimeter during the main period. $n_{3}{ }^{i}$ and $n_{3}{ }^{f}$ are moles of water vapor present in the calorimeter at times $t_{i}$ and $t_{f}$, respectively. $n_{3}{ }^{i}-n_{3}^{f}$ was assumed to be negligible in comparison to $n_{7}$. 
$\Delta H_{v}$ was calculated using

$$
\Delta H_{v}=n_{7} 44.011 \mathrm{~kJ} \cdot \mathrm{mol}^{-1}
$$

which assumes the solutions in eq. 9a are dilute.

The value for $n_{7}$ was first calculated in the shortcut procedure from the values $n_{10}$, the moles of water produced per gram RDF-4, given in table 3 and $n_{10}-n_{7}$ calculated by eq. 11:

$$
n_{10}-n_{7}=\Delta w(18.0154 \cdot m)
$$

$\Delta \mathrm{W}$ is the increase of the mass of the water trap and $\mathrm{m}$ is the mass of the RDF-4 sample. The quantity of water vaporized during the final drift period in experiments 1 through 9 was neglected since the inlet oxygen was saturated. In experi-

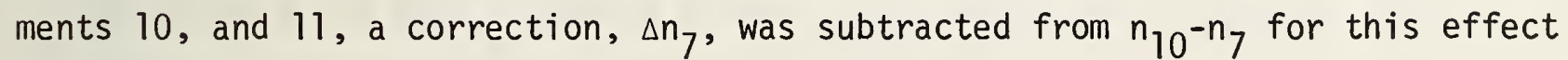
since the inlet oxygen was dry. $\Delta n_{7}$ was calculated from:

$$
\Delta n_{7}=\left(k-k_{c}\right)\left(R_{f}-R_{j}\right) E \Delta t_{f} /(44011 \cdot m)
$$

In eq. $12, k$ is the observed cooling constant and $k_{c}$ is the average cooling constant for runs 1 through $9 . R_{f}$ and $R_{j}$ are the resistances of the platinum thermometer at the end $(f)$ and beginning ( $i$ ) of the main period. $E$ is the energy equivalent of the calorimeter in units of $J \cdot \Omega^{-1} . \Delta t_{f}$ is the length of the final drift period in minutes. Again, $m$ is the mass of the RDF-4 sample. The values of $n_{10^{-n}}$ are 1 isted in column two of table 5 .

The values of $n_{10}$ for Lot $A$ and Lot $B$ were checked in experiments $10 A$ and 11B, respectively, by passing the product gases through a drying trap consisting of tubes containing anhydrous $\mathrm{CaSO}_{4}$ (Drierite) instead of the water bubbler. Since the inlet oxygen was dry in these flow experiments, $n_{7}$ can be determined directly from the increase in mass of the drying trap and $n_{10}$ was calculated from $n_{7}$ plus the increase in the mass of the water condenser in the calorimeter. The results were 
$\mathrm{n}_{10}$ (flow expts.), mmol

$\mathrm{n}_{10}($ table 3$), \mathrm{mmol}$
$26.6 \pm 0.4$

$32.7 \pm 0.5$
$26.9+0.4$

$26.8 \pm 0.5$

The uncertainty in $n_{10}$ determined in the flow experiments is an estimate. The uncertainty in $n_{10}$ from table 3 is based on the imprecision at the $95 \%$ confidence level of the four measurements of the hydrogen content for each log given in table 2 . The two values for Lot $B$ agree within their combined uncertainties but those for Lot $A$ do not. The value of $n_{10}$ measured for Lot $A$ in the flow experiments was taken as the correct value for reasons given below.

First, the efficiency of the water trap, calculated as $\left(n_{10}-n_{7}\right) / n_{10}$, should be independent of whether the sample that is being burned is from Lot A or Lot B. Comparison of the entries in the third column of table 5 (e.g. compare $1 A$ with $2 B$, $4 A$ with the mean of $3 B$ and $5 B$, the mean of $6 A$ and $7 A$ with the mean of $8 B$ and $9 B$, and $10 A$ with $11 B$ ) shows the trap efficiency is consistently lower for Lot $A$ as compared to Lot $B$. Hence, $n_{10}$ for Lot $A$ is too large. An equivalent and clearer result of demonstrating the same result is to assume that, in experiments 1 through 9 , the pressure of water vapor, $\mathrm{P}\left(\mathrm{H}_{2} \mathrm{O}\right.$, out $)$, in the gases leaving the calorimeter is equal to the vapor pressure at the temperature $T_{\text {ex }}$ (see section 3.5). Then, the pressure, $P\left(\mathrm{H}_{2} \mathrm{O}, \mathrm{in}\right)$, of water vapor in the gases entering the calorimeter can be calculated from $n_{7}{ }^{*} P\left(H_{2} 0\right.$, in $)$ should be near the vapor pressure, $P_{\text {sat }}$, of water at room temperature. Values for $\mathrm{P}\left(\mathrm{H}_{2} \mathrm{O}\right.$, out $), \mathrm{P}_{\left(\mathrm{H}_{2} \mathrm{O}\right.}$ in $)$, and $\mathrm{P}_{\text {sat }}$ are listed in columns 4 through 6 of table 5. Comparison of columns 5 and 6 shows that $P\left(H_{2} \mathrm{O}\right.$, in $) / P_{\text {sat }}$ is near one for Lot $B$ but is about $2 / 3$ for Lot $A$. If $n_{10}$ for Lot $A$ is changed from 32.6 to $27.6 \mathrm{~mol}$, the trap efficiencies and $P\left(\mathrm{H}_{2} 0\right.$, in $) / P_{\text {sat }}$ of Lot $A$ and Lot $B$ are about the same. This can be seen from the revised trap efficiencies and pressure ratios given in the parentheses next to columns 3 and 4 of table 5 , respectively.

*

$$
\begin{aligned}
& \left.\mathrm{P}\left(\mathrm{H}_{2}\right) \text {, in }\right) \text { was calculated from } \\
& \qquad \mathrm{P}\left(\mathrm{H}_{2} \mathrm{O}, \text { in }\right) \frac{V}{\mathrm{RT}}+\mathrm{n}_{7}=\left(\mathrm{PH}_{2} \mathrm{O} \text {, out }\right) \frac{V}{\mathrm{R} T} \mathrm{ex} ; \mathrm{V}=\mathrm{V}\left(\mathrm{t}_{\mathrm{f}}-\mathrm{t}_{i}\right) ;
\end{aligned}
$$

$V$ is the measured total volume flow rate. 
๑

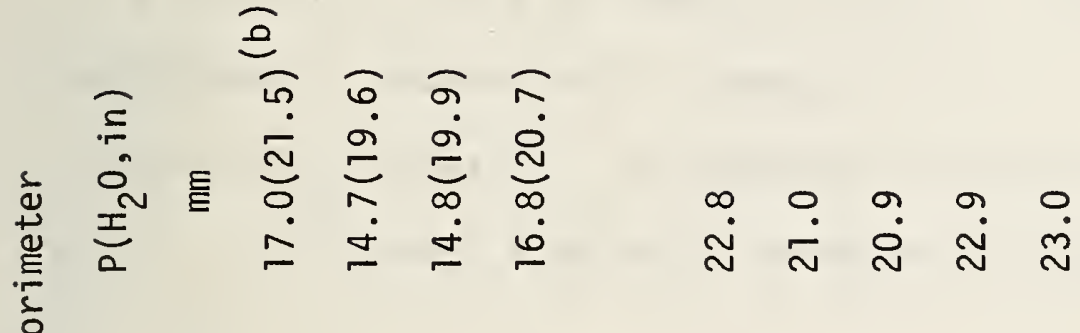

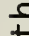

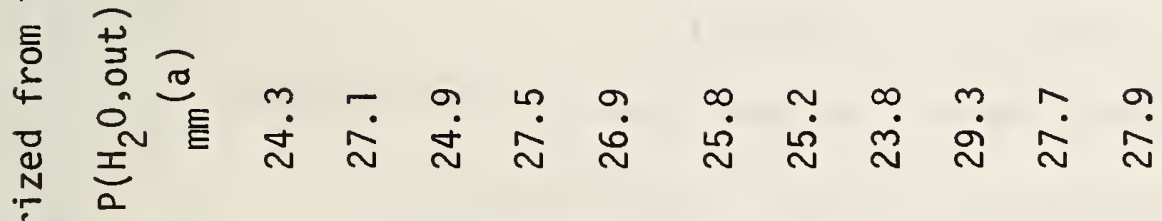

응

㐫 ते

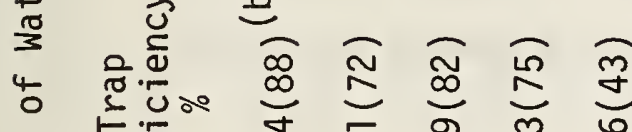

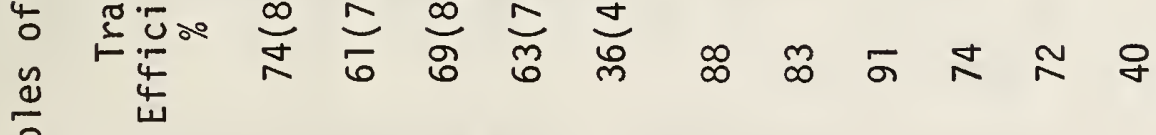

4

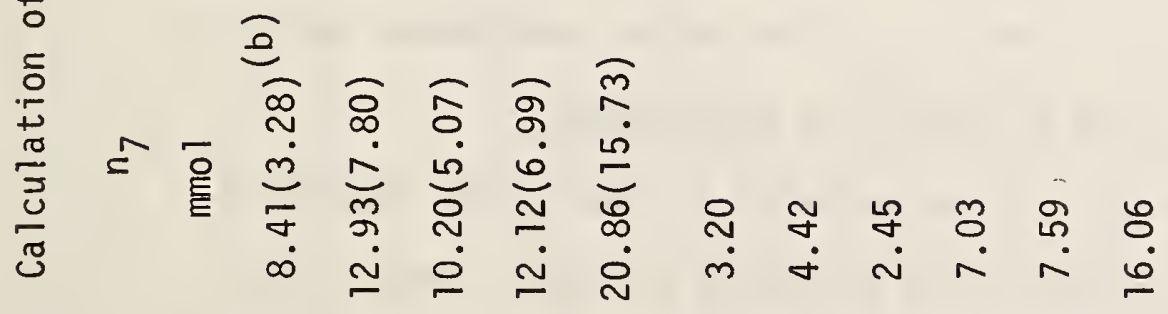

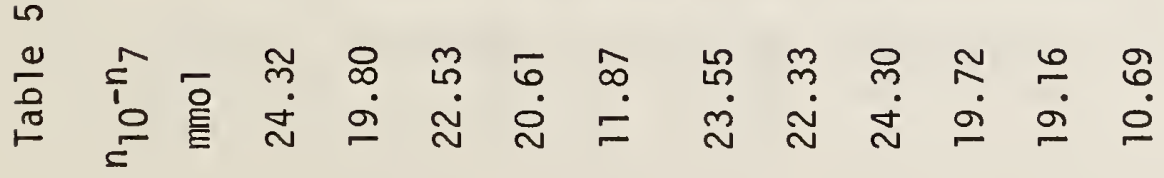

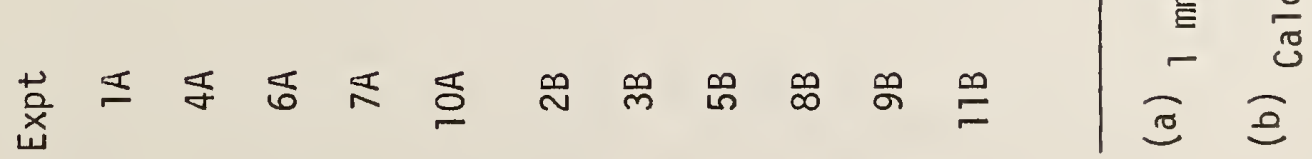


The second reason for adopting $27.6 \mathrm{mmol}$ for $n_{10}$ of Lot $A$ was that a redetermination of the hydrogen content of the remaining portion of Lot $A$ (part of quarter 2, see table 2) gave a value for $n_{10}$ of $25.0 \pm 3.5 \mathrm{mmol}$. This agrees with the result of experiment 10A but disagrees with the values listed in table 3. The carbon content of the same part of Lot A was $39.79 \pm 5.39$ mass \% (dry basis) which agrees with that given in table 3, $43.21 \pm 1.03$ mass $\%$. Thus, segregation cannot account for the discrepancy in the hydrogen concentration.

Revised values for the mass percent of hydrogen, $n_{1}, \Delta n$, and $\Delta H_{s t}$ for Lot $A$ based on $n_{10}=27.0 \mathrm{mmol}$ are given in parentheses of tables 2 through 4 .

\subsection{Calculation of $\Delta \mathrm{H}_{i g n}$}

$\Delta H_{i g n}$ was assumed to correspond to the change in state

$$
(7 / 2) \mathrm{O}_{2}(\mathrm{~g})+\mathrm{Fe}(\mathrm{c})=(1 / 2)\left[\mathrm{Fe}_{2} \mathrm{O}_{3}(\mathrm{~s})+\mathrm{Fe}_{3} \mathrm{O}_{4}(\mathrm{~s})\right]
$$

Eq. 13 assumes no interaction with the ash and that fuse wire is completely oxidized. $\Delta H$ for eq. 13 was taken as $6.66 \mathrm{~kJ} \cdot \mathrm{g}^{-1}$ from reference 4 .

\subsection{Summary of Calculations}

Table 6 lists the values of moles per gram of RDF-4 of $\mathrm{CO}(\mathrm{g}), \mathrm{n}_{4}$, excess oxygen entering the calorimeter, $n_{2}$, moles of water vapor entering the calorimeter, $n_{3}$, and net moles of water vaporized, $n_{7}$.

Table 7 summarizes the calculations of $E$ and $-\Delta H_{s t}$. The estimated value of $\Delta H_{i c}$ for experiment $5 B$ is the average $\Delta H_{i c}$ of the other experiments with Lot $B$. 4.0 Systematic Error Analys is of 25 Gram Flow Experiments.

The larger systematic errors in the calculated results are identified and their magnitudes are estimated in the following sections. Most of the estimates are based on auxiliary experimental measurements which are also described. 


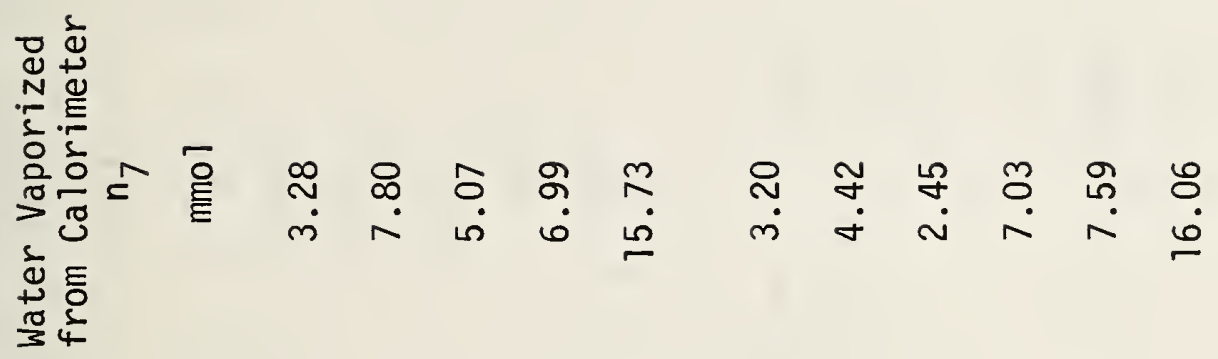

ב

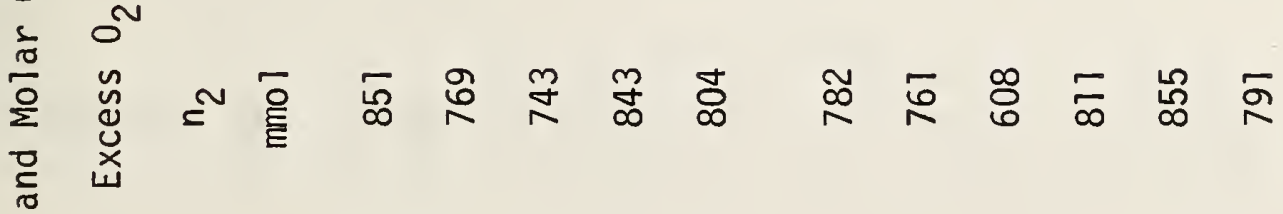

ñ

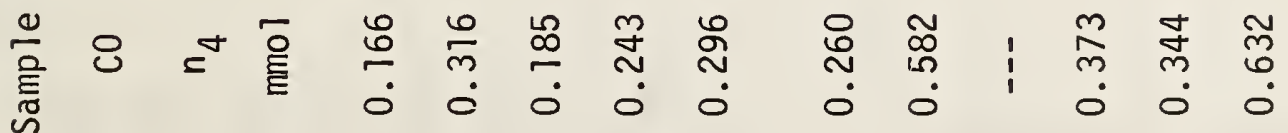

$\omega$

$\frac{0}{\circ} \stackrel{0}{\circ}$ i

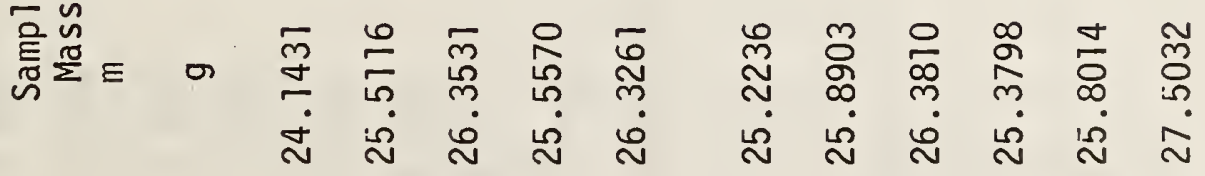

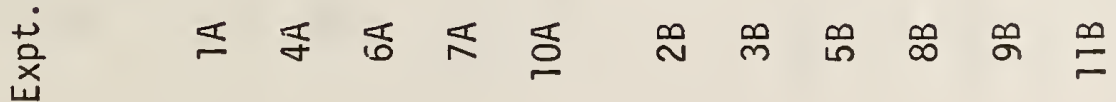


山

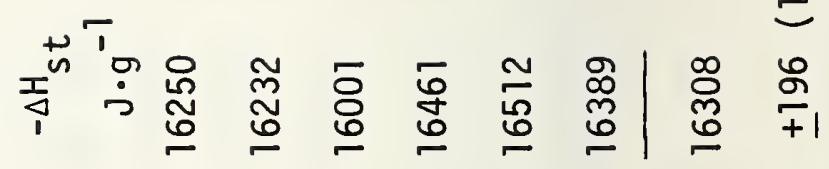

TЕ "в

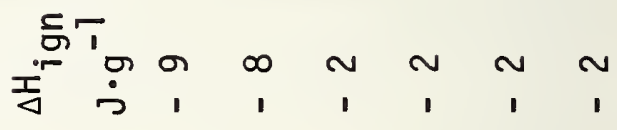

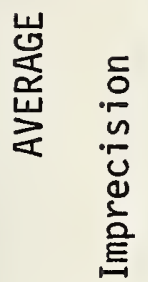

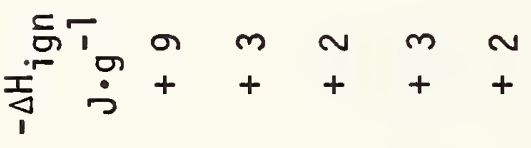

김

는

국

זั่

오

4

홍

둥

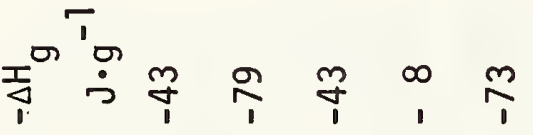

㿝

$\frac{\pi}{3}$

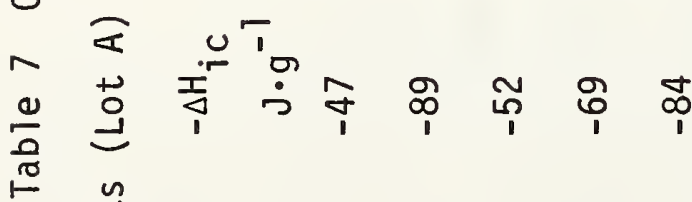

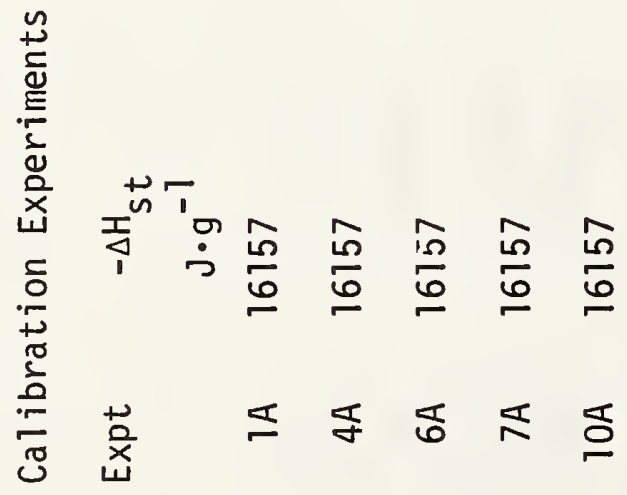

๑

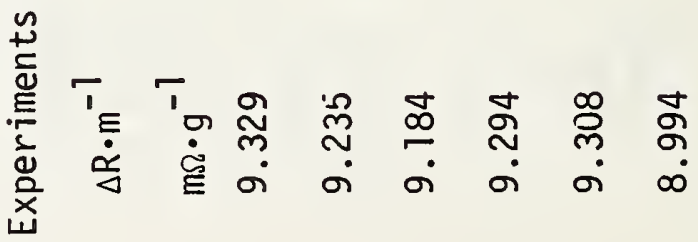

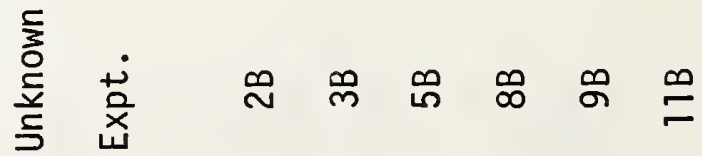




\subsection{Errors in $\Delta R$}

The errors in $k$ and $R_{\infty}$ caused by allowing gases to enter and leave the calorimeter during drift periods are negligible so long as no water is vaporized from the calorimeter. (The estimated error in $k$ is $0.03 \times 10^{-4} \mathrm{~min}^{-1}$.) The calculations assume that no water is vaporized from the calorimeter during the final drift period when the inlet oxygen was saturated. To estimate the error caused by deviations from this assumption, $k$ of eq. 3 was determined when no gases were allowed to enter or leave the calorimeter. The validity of eq. 3 was also checked. This was done as follows.

An electrical heater was installed in the provisional calorimeter and a series of drift rates, $d R / d t$, were determined for eight values of $R$ between 27.99 and $29.56 \Omega$ without opening the calorimeter. Measurements were made over a period of two days at a constant room temperature of $24.4{ }^{\circ} \mathrm{C}\left(\mathrm{R}_{r}\right.$ defined in table 1 was $28.031 \Omega$ ). Provided drift rates were determined thirty or more minutes after the heater was turned off, all the values of $d R / d t$ lay on a straight line with a standard deviation of $0.4 \times 10^{-5} \Omega / \mathrm{min}$. A least square fit of the data gave

$$
(\mathrm{dR} / \mathrm{dt}) \times 10^{5}=6.22 \pm 0.72-(36.1 \pm 2.8)(\mathrm{R}-28)
$$

Uncertainties are imprecisions at the $95 \%$ confidence level for eight measurements.

The results indicate that eq. 3 is valid in the preliminary flow experiments. The results also show that effect of vaporization of water during the final drift period on $k$ and $R_{\infty}$ in experiments 1 to 9 cannot be neglected since the average $k$ for these experiments is $5.69 \times 10^{-4} \mathrm{~min}^{-1}$. Correction of all experiments using eq. 4 with $k_{C}$ equal to $3.6 \times 10^{-4}$ min $^{-1}$ increases $E$ by $0.5 \%$ and decreases $-\Delta H_{s t}$ for lot $B$ by $0.6 \%$. To be consistent, an additional correction must be done on $\Delta H_{V}$ since vaporization of water cannot be neglected during the final drift period when the inlet oxygen is saturated. The correction is estimated in section 4.5. The combined effect is to increase $E$ by $0.7 \%$ and decrease $-\Delta H_{\text {st }}$ for Lot $B$ by $0.7 \%$. 


\subsection{Errors in $\Delta H_{s t}$}

The measured millequivalents of acid per gram (meq $\cdot \mathrm{g}^{-1}$ ) of RDF-4 sample contained in the water trap are listed in the first column of table 8 . From table 3 , one would expect 0.08 for Lot $A$ and 0.10 for Lot B. Estimated correction of the experimental results for vaporization of acid (by multiplying each by $n_{10} /\left(n_{10}-n_{7}\right)$ from table 5) yields the results given in the second column of table 8. The measured value of the acid content of the water in the bubbler through which the cumulative product gases of experiments 6 through 9 were passed was $0.064 \mathrm{meq} \cdot \mathrm{g}^{-1}$. This is in reasonable agreement with the estimated value of $0.073 \mathrm{meq} \cdot \mathrm{g}^{-1}$ which was computed as the sum of the values in the third column in table 8 minus the sum of those in the second column for these experiments. However, since the values given in the second column of table 8 for experiments 10 and 11 grossly exceed the predicted results, the agreement must be regarded as fortuitous. We concluded that 1) most of the oxidized sulfur is $\left.\mathrm{SO}_{2}(\mathrm{~g}), 2\right)$ most of the aqueous acid is probably $\mathrm{HCl}$, and 3) the correction for the small but unknown amount of aqueous $\mathrm{H}_{2} \mathrm{SO}_{4}$ is negligible.

The possibility that the chemical composition of the ash produced in the bomb reaction is different from that of the flow reaction is not reflected in differences in the mass fraction of the ash contents. The mass fraction of the ash determined by weighing of the residue in the bomb experiments, flow experiments, and ASTM test methods corrected to the moisture contents of the flow work are given in Table 8. 
Table 8 Acid Content of Water Trap

\begin{tabular}{|c|c|c|}
\hline Expt. & $\begin{array}{l}\text { Acid } \\
\mathrm{meq} \cdot \mathrm{g}^{-1}(\mathrm{a})\end{array}$ & $\begin{array}{c}\text { Acid/Trap Efficiency (b) } \\
\text { meq } \cdot g^{-1}\end{array}$ \\
\hline $1 A$ & --- & -- \\
\hline $4 A$ & 0.069 & 0.098 \\
\hline $6 \mathrm{~A}$ & 0.076 & 0.094 \\
\hline $7 A$ & 0.073 & 0.099 \\
\hline $10 \mathrm{~A}$ & 0.066 & 0.154 \\
\hline $2 B$ & 0.066 & 0.075 \\
\hline $3 B$ & 0.064 & 0.077 \\
\hline $5 B$ & --- & -.- \\
\hline $8 B$ & 0.037 & 0.051 \\
\hline $9 B$ & 0.038 & 0.053 \\
\hline $11 \mathrm{~B}$ & 0.069 & 0.172 \\
\hline
\end{tabular}
(a) meq = milliequivalents
(b) See table 5 
ASTM Test (a)

Combustion Bomb

Flow Calorimeter
$0.2322 \pm 0.0148$

$0.2363 \pm 0.0035$

$0.2301 \pm 0.0028$

Uncertainties are imprecisions at the $95 \%$ confidence level. The results agree within their imprecisions.

\section{(a) ASTM Test D3173-73}

A check was made for possible changes or inhomogeneity in the RDF-4 samples between the time when the original composition determinations and bomb calorimetric experiments were made and flow calorimetric experiments were carried out. After the flow experiments, ash and moisture contents of each lot were determined before and after mixing each lot in a vee-blender. The mass fraction results of the ASTM test were as follows:

Lot $A$

Before mixing

After mixing
0.2135

0.0469

0.2133

0.0464
Lot B

0.2317

0.0369

0.2215

0.0354

Ash contents have been corrected to the moisture contents of the flow work for comparison with previous results. The only significant difference is in the Lot B ash content which indicates that some segregation of Lot B may have occurred. This possibility is also reflected in the large uncertainty associated with the original ash determinations on Lot B (see above). Differences between these moisture contents and those used in the flow work (i.e. 0.0508 for Lot $A$ and 0.0396 for Lot B) suggested the assignment of an estimated systematic error of 0.005 to the mass fraction of moisture for each lot in the flow work. 


\subsection{Errors in $\Delta H_{i c}$}

Maximum errors of $5 \%$ in the gas flow rate and $4 \%$ in the fractional content of CO in the product gases produce errors of $0.03 \%$ and $0.02 \%$, respectively, in $E$. The net effect on $-\Delta H_{s t}$ for Lot $B$, if the error is the same in all experiments, is negligible.

The average loss in mass of the ash from the flow experiments due to heating with the flame of an oxygen-gas torch was $0.071 \%$ of the initial sample mass. The species which vaporized or were oxidized were not identified. After several experiments, black smudges resembling "soot" were observed on the quartz plate and/or crucible; these disappeared upon flaming.

The residual carbon content of the heated ash was determined to be $0.008 \%$ of the initial sample mass.

Traces of brownish material resembling "tars" were found on the exit port of the thermal shield after each combustion run, the mass was less than $0.001 \%$ of the initial sample mass.

The total solid products of incomplete combustion constitute $0.08 \%$ of the initial sample mass. Treated as unburned combustibles in RDF-4, E would increase by $0.10 \%$. If the solids are treated as pure carbon, $E$ increases by $0.14 \%$. In either case the net effect on $-\Delta H_{s t}$ for Lot $B$ is negligible.

\subsection{Errors in $\Delta H_{9}$}

A maximum error of $5 \%$ in the inlet gas flow rate introduces an error of $0.02 \%$ in $E$. Combined with the flow rate effect on $\Delta H_{i c}$, a $5 \%$ error in flow rates introduces an error of $0.05 \%$ in $E$. The net effect on $-\Delta H_{s t}$ for the same error in all experiments is negligible.

Measurement of $T_{\text {ex }}-T_{r}$ and $T_{i n}-T_{r}$, where $T_{r}$ is the room temperature were made in experiments $5,6,9$, and 11 using Type $K$ thermocouples. Analys is of the data showed that during drift periods eqs. 14 apply. 


$$
\begin{aligned}
& T_{\text {in }}-T_{r} \simeq-0.17+0.26\left(T-T_{r}-0.17\right) \\
& T_{e x}-T \simeq-0.13\left(T-T_{r}-0.17\right)
\end{aligned}
$$

$T$ is the observed temperature of the calorimeter water. Deviations from eq. 14b during the main period were less than $0.1 \mathrm{~K}$ except for temporary changes in thermocouple voltage that seem to be associated with evaporation and/or condensation of moisture on the thermocouple junction. The uncertainty in the expressions on the right side of eqs. 14 is unknown but appreciable. In any event, recalculation of $\Delta \mathrm{H}_{\mathrm{gl}}$ for all experiments using eqs. 14 increases $E$ by $0.18 \%$ and decreases $-\Delta H_{\text {st }}$ for Lot $B$ by $0.26 \%$.

\subsection{Errors in $\Delta \mathrm{H}_{\mathrm{V}}$}

The correction for the error made in assuming that the water vaporized during the final drift period is negligible when the inlet oxygen is saturated was calculated using eq. 10 and the drift data given in section 4.1. E increases by $0.17 \%$ and $-\Delta H_{\text {st }}$ for Lot B decreases by $0.13 \%$.

\subsection{Summary of Error Analysis}

A summary of the effect of the errors expressed in terms of their percentage effect on the energy equivalent, $E$, and the energy of combustion, $-\Delta H_{s t}$, of Lot $B$ is given in table 9. The parentheses after the error source enclose the section number where the analysis is given. Below each error source, variation contributions are itemized. The percent effect of each error contribution to $-\Delta H_{s t}$, given in column three, was computed additively.

The total systematic error in $-\Delta H_{s t}$ is $-1.0 \pm 1.0 \%$. The systematic error associated with the provisional calorimetric assembly is considered to be $-2.6 \%$ (item $4 . b$ ). 
Table 9. Systematic Error Analysis of 25 Gram Flow Experiments

\section{EFFECT}

Source of Error

$\begin{array}{cc}E & -\Delta H_{s t} \\ (\%) & (\%)\end{array}$

1. $\Delta R(4.1)$

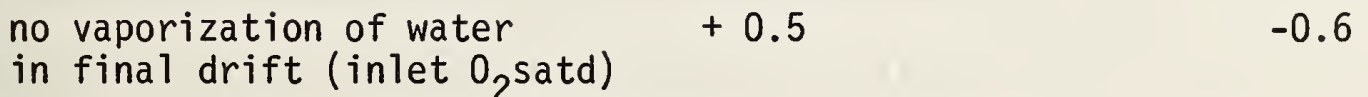

2. Sample mass

moisture content

$$
\pm 0.5
$$

3. $\Delta H_{i c}(4.3)$
a) Co concentration (a)
$< \pm 0.02$
0.0
b) flow rate ${ }^{(b)}$ :
$< \pm 0.03$
0.0
c) complete combustion
$+0.1$
0.0
to $\mathrm{CO}$ and $\mathrm{CO}_{2}$

4. $\Delta H_{g}(4.4)$
a) flow rate ${ }^{(b)}$
$\leq \pm 0.02$
0.0
b) $T_{\text {in }}=T_{r}, T_{\text {ex }}=T$
$+0.18$
$-0.26$

5. $\Delta H_{v}(4.5)$

no vaporization of water in final drift

(inlet $\mathrm{O}_{2}$ satd)

(a) Assumes error in CO concentration of $\pm 4 \%$.

(b) Assumes error in product gas flow rate of $\pm 5 \%$. 
5. Results of 25 Gram Flow Experiments and Conclusions

A summary of the results is listed in table 10. In the calibration experiments, $\Delta H_{s t}$ is the enthalpy change for the standard flow reaction for one gram of RDF-4 calculated from the results of combustion bomb measurements. Results are expressed in terms of the mass of sample with moisture contents of the actual calibration experiments. $\Delta \mathrm{H}_{\mathrm{ic}}$ is the average correction per gram of RDF-4 for incomplete combustion of all $\mathrm{CO}$ to $\mathrm{CO}_{2} \cdot \Delta \mathrm{H}_{\mathrm{g}}$ is the average correction per gram of RDF-4 for the net heat transport by oxygen entering and gaseous products leaving the calorimeter at some temperature different than the temperature of the standard flow reaction (we assumed this temperature to be the initial calorimeter temperature). $\Delta H_{v}$ is the average correction for the heat of vaporization of water formed in the combustion that is lost from the calorimeter. $\Delta H_{i g n}$ is the average of the ratio of the enthalpy of the combustion of the iron fuse wire used to ignite the RDF-4 sample divided by the mass of the RDF-4 sample. $\Delta R / m$ is the average ratio of temperature rise, expressed in ohms (i.e. $0.1 \Omega \sim 1^{\circ} \mathrm{C}$ ), of the calorimeter corrected for heat leak divided by the mass of the sample, $m$.

$E$ is the mean energy equivalent of the calorimeter and is calculated from the basic measurement equation:

$$
\frac{E \Delta R}{m}=-\left(\Delta^{\prime \prime} s t+\Delta H_{i c}+\Delta H_{g}+\Delta H_{v}+\Delta H_{i g n}\right)
$$

The imprecision is the product of the standard deviation of the mean and the "Student $t$ " factor for four degrees of freedom at the $95 \%$ confidence level. 
Table 10 Summary of 25 Gram Flow Results

Calibration Experiments (Lot $A$ )

$\begin{array}{ccc}+\Delta H_{s t} & -16157 \mathrm{~J} \cdot g^{-1} & \left(-6946 \mathrm{BTU} \cdot 1 \mathrm{~b}^{-1}\right) \\ +\Delta \mathrm{H}_{i \mathrm{C}} & +68 \mathrm{~J} \cdot \mathrm{g}^{-1} & \left(+29 \mathrm{BTU} \cdot 1 \mathrm{~b}^{-1}\right) \\ +\Delta \mathrm{H}_{\mathrm{g}} & +49 \mathrm{~J} \cdot \mathrm{g}^{-1} & \left(+21 \mathrm{BTU} \cdot 1 \mathrm{~b}^{-1}\right) \\ +\Delta \mathrm{H}_{v} & +340 \mathrm{~J} \cdot \mathrm{g}^{-1} & \left(+146 \mathrm{BTU} \cdot 1 \mathrm{~b}^{-1}\right) \\ +\Delta \mathrm{H}_{i g n} & -4 \mathrm{~J} \cdot \mathrm{g}^{-1} & \left(-2 \mathrm{BTU} \cdot 1 \mathrm{~b}^{-1}\right) \\ \Delta \mathrm{R} \cdot \mathrm{m}^{-1} & 9.156 \mathrm{~m} \Omega \cdot \mathrm{g}^{-1} & \left(4.153 \Omega \cdot 1 \mathrm{~b}^{-1}\right) \\ \mathrm{E} & 1.7151 \mathrm{MJ} \cdot \Omega^{-1} & \left(1608 \mathrm{BTU} \cdot \Omega^{-1}\right)\end{array}$

imprecision

$$
\pm .0156(0.91 \%) \quad \pm 15
$$

Unknown Experiments (Lot $B$ )

\begin{tabular}{|c|c|c|}
\hline$\Delta R \cdot m^{-1}$ & $9.224 \mathrm{~m} \Omega \cdot \mathrm{g}^{-1}$ & $\left(4.184 \Omega \cdot 1 \mathrm{~b}^{-7}\right)$ \\
\hline$E \Delta R \cdot m^{-1}$ & $+15,820 \mathrm{~J} \cdot \mathrm{g}^{-1}$ & $\left(+6801 \mathrm{BTU} \cdot 1 \mathrm{~b}^{-1}\right)$ \\
\hline$\Delta \mathrm{H}_{\mathrm{ic}}$ & $+\quad 124 \mathrm{~J} \cdot \mathrm{g}^{-1}$ & $\left(+\quad 53 \mathrm{BTU} \cdot 1 \mathrm{~b}^{-1}\right)$ \\
\hline$\Delta \mathrm{H}_{\mathrm{g}}$ & $69 \mathrm{~J} \cdot \mathrm{g}^{-1}$ & $\left(+\quad 30 \mathrm{BTU} \cdot 1 \mathrm{~b}^{-1}\right)$ \\
\hline$\Delta H_{v}$ & $297 \mathrm{~J} \cdot \mathrm{g}^{-1}$ & $\left(+128 \mathrm{BTU} \cdot 1 \mathrm{~b}^{-1}\right)$ \\
\hline$\Delta \mathrm{H}_{\text {ign }}$ & $4 \mathrm{~J} \cdot \mathrm{g}^{-1}$ & $\left(-\quad 2 \mathrm{BTU} \cdot 1 \mathrm{~b}^{-1}\right)$ \\
\hline$-\Delta H_{s t}$ & $16,308 \mathrm{~J} \cdot \mathrm{g}^{-1}$ & $\left(7011 \mathrm{BTU} \cdot 1 \mathrm{~b}^{-1}\right)$ \\
\hline imprecision & $\pm 196(1.20 \%)$ & \pm 84 \\
\hline
\end{tabular}

overali

imprecision $\pm 238(1.46 \%) \quad \pm 102$ 
The results of the experiments on the "unknown" are given in the bottom half of table 10. Definitions are the same except: 1) results are expressed in terms of the sample mass having a moisture content of the unknown experiments; 2) $-\Delta H_{s t}$ for Lot $B$ is calculated from eq. 15 using the mean value of E determined from the calibration experiments; and 3) the precision of $-\Delta H_{s t}$ is calculated using the "Student $t$ " factor for five degrees of freedom at the $95 \%$ confidence level. The overall imprecision in $-\Delta H_{\text {st }}$ for Lot $B$ takes into account the imprecision in $E$ from the Lot $A$ experiments. The value of $-\Delta H_{s t}$ for Lot $B$ was $16,308 \mathrm{~J} \cdot \mathrm{g}^{-1}\left(7011 \mathrm{Btu} \cdot 1 \mathrm{~b}^{-1}\right)$. The value of $-\Delta \mathrm{H}_{\mathrm{st}}$ is calculated from bomb calorimetric measurements for Lot $B$ was $16,597 \mathrm{~J} \cdot \mathrm{g}^{-1}\left(7135 \mathrm{Btu} \cdot 1 \mathrm{~b}^{-1}\right)$.

The flow and bomb calorimeter results for measurements on Lot $A$ and Lot $B$ are summarized in Table 11.

Table 11. Comparison of Flow and Bomb Results Flow Bomb

Imprecision, Lot A Measurements $0.91 \%$ $0.85 \%$ Imprecision, Lot B Measurements $1.20 \%(a)$ $0.46 \%$

$r=\left(\Delta H_{s t}\right)_{B} /\left(\Delta H_{s t}\right)_{A}$ 1.009 1.027 Uncertainty in $r$ $0.027^{(b)}$ 0.010

\footnotetext{
(a) Excludes imprecision in E.

(b) Sum of imprecision and the systematic error due to moisture content of samples.
} 
The imprecision of the flow measurements on lot $A$ and lot $B$ were $0.91 \%$ and $1.21 \%$ respectively. (i.e., imprecision of $E$ and $-\Delta H_{s t}$ excluding that due to $E$, respectively). The corresponding imprecision in the calorific values determined with the bomb calorimeter for four samples of lot A was $0.85 \%$ and for four samples of lot B was $0.46 \%$. The ratio, $r$, of $\Delta H_{s t}$ of lot $B$ divided by that of lot $A$ for the flow measurements is 1.009 with an imprecision of 0.015 (i.e. $1.5 \%$ ) at the $95 \%$ confidence level. For purposes of comparison with bomb results, the uncertainty of $r$ is estimated to be \pm 0.027 . This was computed as the sum of the overall imprecision in $r$ plus twice the uncertainty in the mass of the sample ( 0.5 mass percent) due to the uncertainty in moisture content of the RDF-4 samples used in the flow work. The corresponding value of $r$ based on the bomb calorimeter data was 1.027 with an imprecision and uncertainty of \pm 0.010 . The flow and bomb measurements agree within their combined uncertainties.

The average corrections for vaporization of water in table 10 are large because of the very large corrections made in experiments $10 A$ and $11 B$, in which the inlet oxygen was dry (rather than saturated with water). The average correction for the two dry inlet oxygen experiments was $4.3 \%$ of $-\Delta H_{\text {st }}$. When the inlet oxygen was asturated with water the average correction for vaporization was $1.6 \%$ for Lot $A$ and $1.3 \%$ for lot $B$. The sum of the average corrections for $C 0$, heat transport by gas flow, and sample ignition were only $0.7 \%$ for Lot $A$ and $1.2 \%$ for Lot $B$.

The average corrections for the heat leak applied to the observed temperature rise were $1.3 \%$ and $2.1 \%$ of $\Delta R$ for $\operatorname{lot} A$ and lot $B$, respectively. The corrections ranged from -0.7 to $+4 \%$ of $\Delta R$. The corrections assume that the temperature of the calorimeter environment is held constant and that steady state heat transfer between the calorimeter water and the environment is achieved rapidly. Since these conditions were not met by the prototype calorimeter, we felt the largest error in the flow measurements would be in the correction for the heat leak. Appropriate experiments plus an error analysis were carried out to determine if this was true. 
An error analysis of the results (see section 4) shows that the total estimated systematic error of the enthalpy ratio, $r$, is between $-2.0 \%$ and $+0.0 \%$ and that the estimated systematic error in $r$ associated with the prototype calorimeter is about $-0.3 \%$. Hence, we expect use of the completed final calorimeter rather than the prototype calorimetric assembly should reduce the systematic error in $r$ to between $-1.7 \%$ and $+0.3 \%$. If the final calorimeter were to be calibrated with cellulose or with an electrical heater, the systematic error would be about the same. A further reduction in the systematic error can only be accomplished in the final calorimeter by changes in the procedures and/or auxiliary measurements used in this preliminary work and further changes in the equipment. These changes will affect future measurements in the final 25 gram capacity flow calorimeter. A summary of the proposed changes and the basis for the proposals is as follows.

1) Heat leak corrections were made assuming no water is vaporized from the calorimeter during the final drift period when the oxygen entering the calorimeter is saturated with water vapor. The estimated maximum correction in $r$ due to the error in this assumption is $-0.7 \%$. The error can be eliminated by allowing no gases to enter or leave the calorimeter during both drift periods.

2) The method used to calculate the correction for water vaporized from the calorimeter during the main period was indirect. It was calculated from the average elemental hydrogen content of each lot of RDF-4 and the amount of water condensed in the water trap in each experiment. A more accurate method is to determine the amount of water vaporized from the calorimeter directly. The accuracy and precision of such a measurement will be greater if the oxygen supplied to the calorimeter is dry. In order to reduce the magnitude of the vaporization correction under this condition, a more efficient water trap is needed and has been constructed. 
3) The results were calculated assuming that all sulfur was oxidized to $\mathrm{SO}_{2}(\mathrm{~g})$ and all chlorine converted to hydrochloric acid. All the acid was assumed to be retained in the water trap. Comparison of the measured and predicted acidity of the water condensed in the water trap (i.e. from the elemental chlorine content of each RDF-4 lot) confirmed this assumption. Measurement of the acidity of the water in the product gas scrubber indicated aqueous acid was leaving the calorimeter. Hopefully, this loss can be reduced by the changes made to the water trap to improve its condensation efficiency. The chloride and sulfur content as well as the total acid content of the water trap must be determined in each experiment.

4) The estimated systematic error in $r$ of $\pm 0.5 \%$ due to possible error in the moisture content in each lot of RDF-4 should be reduced by changes in procedure.

5) The reduction in the mass of the ash produced by heating the ash plus crucible and plate with the flame of an oxygen-gas torch corresponded, on the average, to $0.07 \%$ of the original RDF-4 sample mass. The estimated systematic error in $r$ is negligible unless the calorimeter is calibrated with cellulose. In the latter case the error is beween $+0.09 \%$ and $+0.14 \%$. We feel the more probable value is $0.09 \%$. However, the species vaporized in the procedure were not identified. A possible method of eliminating the error is to expose the ash, sample plate, and crucible to a methane-oxygen flame during the last stages of combustion.

We found that the amount of ash formed in the combustions in the flow calorimeter agreed with that determined in the bomb calorimetric measurements and by the ASTM method within their combined experimental imprecision $( \pm 3.5 \%$ for the flow work and $\pm 2 \%$ for the bomb and ASTM work). (Measurement of ash contents for each lot before and after homogenization in a vee-blender suggested some segregation within Lot B may have occurred, but not Lot A. Agreement between the 
amount of ash in the bomb and flow experiments observation is a necessary but not sufficient condition that the enthalpy of reaction in a combustion bomb and flow calorimeter are the same. A subse̋quent series of measurements in the final flow calorimeter will be needed to relate the bomb and flow measurements .

6. References

[1] Kirklin, D.R., Domalski, E.S., and Mitche11, D.J., NBSIR 80-1968.

[2] Prosen, E.J., Maron, F.W., and Rossini, F.D., J. Res. Nat. Bur. Std. $\underline{42}$, 269 (1949).

[3] Goldberg, R.N., Nuttal1, R.L., Prosen, E.J., and Brunetti, A.P. Digital Data Acquisition and Computer Computation Applied to Calorimetric Experiments. Nat. Bur. Stand. (U.S.) Report 10-437; 1971 June.

[4] Wagman, D.D., Evans, W.H., Parker, V.B., Halow, I., Bailey, S.M., and Schumm, R.H., Selected Values of Chemical Thermodynamic Properties. Nat. Bur. Stand. (U.S.) Tech. Note 270-3; 1968 January. 264 p. 


\section{Acknowledgements}

The authors wish to acknowledge the very substantial contributions made by others at NBS to this work: W. Dorko and R. Burke (and colleagues) of the Center for Analytical Chemistry for mass spectrometric and elemental analyses, respectively, of combustion products; S. Abramowitz of the Center for Chemical Physics for infrared spectroscopic analysis of the combustion product gases and for guidance along with $W$. Dorko in selection of instrumentation for analysis of product gases; J. Berke, of the Office of Recycled Materials, for photographing combustions in the combustor development experiments; R.V. Kelly of the Center for Chemical Physics who determined ash and moisture contents of the RDF-4 samples after the flow combustion runs; G.T. Armstrong, D. Garvin, and E.J. Prosen of the Chemical Thermodynamics Division who provided key ideas to the 25 gram combustor development; and E.J. Prosen for advice on flow calorimetry.

This work was jointly sponsored by the NBS Office of Recycled Materials and the U. S. Department of Energy, Office of Energy from Municipal Waste. 

Part C. TRIAL COMBUSTIONS OF KILOGRAM-SIZE SAMPLES OF MUNICIPAL SOLID WASTE

A.E. Ledford, R.V. Ryan, M.L. Reilly, E.S. Domalski, and K.L. Churney

\section{Abstract}

A new calorimeter is being developed at the National Bureau of Standards to determine the enthalpies of combustion of kilogram-size samples of municipal solid waste (MSW) in flowing oxygen near atmospheric pressure. Experiments were carried out to develop a prototype combustor in which pellets of relatively unprocessed MSW can be rapidly and completely burned with minimal scattering of ash. Pellets of up to $2.2 \mathrm{~kg}$ mass with ash contents between 20 and 35 percent have been successfully burned at a rate of 15 minutes per kilogram initial mass with $\mathrm{CO} / \mathrm{CO}_{2}$ ratios not greater than 0.1 percent.

\section{Introduction}

The Resource Conservation and Recovery Act of 1976 mandates the National Bureau of Standards (NBS) to publish guidelines for the development of specifications for the classification of materials which can be recovered and would otherwise be destined for disposal. Of particular national interest is the desire to produce useable energy from such sources as refuse and refusederived-fuel (RDF).

At present, the calorific value of RDF is determined at commercial laboratories with conventional combustion bomb calorimetric techniques using gramsize samples. Such samples are prepared by reducing the bulk RDF to a powder of $0.5 \mathrm{~mm}$ particle size (RDF-4). Substantial processing is required to obtain the gram-size sample and there is concern among some combustion engineers that this processing may significantly alter its calorific value. There are also questions about sampling procedures and how representative such a gram-size 
sample can be of the waste stream.

To address these doubts, NBS has developed a calorimeter to determine the calorific value of kilogram-size samples of dried RDF-2. RDF-2 is municipal solid waste which has been processed to reduce the particle size so that 95 mass-percent passes through a $15 \mathrm{~cm}$ square mesh screen. Kilogram-size samples of RDF-2 should represent the properties of the heterogeneous bulk material more accurately and reliably than the highly processed gram-size samples currently used in bomb calorimetric determinations.

For safety considerations, we chose to build a calorimeter for the combustion of samples in flowing oxygen near atmospheric pressure rather than to scale up the conventional high pressure oxygen combustion bomb. Unfortunate$1 y$, after the 1880 's the development of the flow technique to determine the enthalpy of combustion of solids was discontinued in favor of the simpler procedures and more quantitative results which could be obtained with the bomb calorimeter. As a consequence, the first goal of the NBS project was to demonstrate that the oxygen flow technique could be used to obtain complete combustion of municipal solid waste (MSW).

A small combustion flow calorimeter $[1,2]$, that has been sucessfully used to burn $25 \mathrm{gram}$ pellets of the highly processed MSW prepared for combustion bomb experiments, was discussed in Part B of this report. The amount of carbon in the ash and the uncertainty in the amount of $\mathrm{CO}$ in the combustion products contributed errors of less than $0.1 \%$ to the enthalpy of combustion.

Before the design of a large-scale combustion flow calorimeter could be undertaken, it was necessary to determine the burning characteristics of the kilogram-size RDF-2 pellets in oxygen. The composition of the kilogram-size pellet is more variable and the physical heterogeneity measured against the sample size is greater than the 25 gram pellet of highly processed RDF-4. As 
a consequence its combustion properties were expected to be different.

The goal of the trial experiments was to develop a method for controlled burning of the sample pellets at a rate of 15 minutes per kilogram or less. This time limit was estimated to be the maximum time which would guarantee that the imprecision contributed by the calorimetric measurements would be less than one percent.

The trial combustor was designed, fabricated, and assembled during the second and third quarters of FY 1980. Suitable instrumentation and equipment to operate and evaluate the performance of the combustor were also set up during this time. Trial combustions using various arrangements of sample and oxygen supply inside the combustor were carried out during the last quarter of FY 1980.

\section{Apparatus and Samples}

Trial combustions of samples up to $2.2 \mathrm{~kg}$ of RDF-2 were carried out in a burner cooled by convective and radiant heat losses to ambient temperature. The burner was mounted in a large exhaust hood. The burner design was similar to that used in the 25 gram experiments in that thermal shields were employed to keep the hot, reacting, combustion product gases from being cooled by contact with the outer burner walls. The combustor differed from that used in the 25 gram experiments in that all of the oxygen was supplied locally to the sample; no oxygen flowed between the thermal shield and the cool combustor walls. 0xygen was supplied in the form of high velocity jets which were directed either at the top of or the side of the sample or both, depending on the experiment. A diffuse, slower, flow of oxygen was directed upward at the bottom of the sample.

The combustor, as it appeared in the first of the trial burns, is shown in cross section in Fig. 1. The unit consists of two chambers. The lower 
chamber, which was the cylindrical region enclosed by the lower burner jacket (E of Fig. 1), contained the sample and two oxygen supply inlets. The sample was supported on vertical stainless steel pins which fit into the holes of a porous stainless steel plate $(H)$. This support system was analogous to the quartz plate of the 25 gram combustor. Primary oxygen was supplied to the bottom of the sample from the inlet I. Secondary oxygen was directed at the top of the sample by three jets aimed radially and horizontally by the inlet F. The intent of the sample-oxygen supply arrangement was to mimic as far as possible the 25 gram flow experiments while maintaining an unobstructed view of the combustion. Hence, no crucible was used.

The thermal shield (B) of the upper chamber and upper burner jacket (C) sat on a annular steel plate (D) which was supported by three rods $(G)$ that projected through the lower chamber to the concrete floor. The annular plate also supported the lower burner jacket which was bolted to the plate around two-thirds of its circumference.

For access to the lower chamber, one-third of the circumference of the lower burner jacket was a semi-cylindrical door. Two Pyrex glass viewing ports (not shown in Fig. 1) were installed in the door so that the course of a combustion could be observed.

Oxygen was supplied to the primary and secondary inlets in the lower burner jacket by independent sources, each consisting of three standard b,200 liter (STP) oxygen tanks equipped with reducing valves and connected in parallel. Flow rates were measured with variable orifice flowmeters. A product gas analysis train similar to that used in the 25 gram experiments was used to monitor the $\mathrm{CO}$ and $\mathrm{CO}_{2}$ production.

Temperatures were monitored at as many as twelve different locations using 18 gauge Type $K$ (Chromel $P$ - Alumel) thermocouples. 
The RDF-2 from which the sample pellets were made was obtained from the Teledyne National Resource Recovery Facility in Cockeysville, Maryland where municipal solid waste of Baltimore County is processed. Lots were withdrawn at random from the conveyer belt leaving the primary shredder. At NBS, the large noncombustibles were removed and the remainder was dried in air at $105^{\circ} \mathrm{C}$ for 12 to 16 hours. Sample pellets were made by compressing the dried RDF-2 in a cylindrical die piece with a force ranging from 265 to $625 \mathrm{kN}$ (30 to 70 tons). A single compression yielded a pellet with reasonably good adhesion of the various heterogeneous horizontal layers. This adhesion was not improved by wetting the material with water prior to pressing. The finished pellet had a diameter of $22 \mathrm{~cm}$ and a height of about $6 \mathrm{~cm} / \mathrm{kg}$ of sample mass. To test the effect of increased surface area, three or seven vertical holes were drilled in some of the pellets using a metal drill and jig to hold the sample. One cellulose pellet was made by pressing pure cellulose fluff using the same technique.

In a11, eighteen experiments were carried out to test the effectiveness of: (1) various arrangements of primary and secondary oxygen inlets, (2) preheating the oxygen, and (3) reducing heat losses from the sample by the use of a crucible and a radiation shield. Thirteen experiments were run with RDF-2 pellets. Five experiments were run with pure cellulose or its equivalent (a stack of unglazed paper plates).

Changes in the apparatus made as a result of these tests are illustrated by Fig. 2 which shows the configuration of the lower chamber of the combustor used in the final two experiments. An RDF-2 sample with seven vertical $2.5 \mathrm{~cm}$ diameter holes sat on a horizontal lattice of alumina rods which rested on a stainless steel support (see D of Fig. 2). Two tiers of secondary oxygen inlets ( $B$ and $C$ ) were aimed radially inward and horizontally at the side of 
the pellet. The lower tier of six inlets was supplied with oxygen that was preheated by passing it through coiled tubing ( $F$ ) which was wound on the outside of the crucible. The upper tier of three inlets was supplied with oxygen that was preheated by passing it through the coil (I) which was inside of the crucible. 0xygen was supplied to the bottom of the sample from the primary oxygen inlet (E). Most of the ash falls through the center of the inlet to the bottom of the crucible. A radiation shield which just fits the inside diameter of the annular steel plate ( $D$ of Fig. 2) has been placed around the crucible to reduce heat losses.

Back flow of the product gases between the crucible side and the lower thermal shield was prevented by a low upward flow of diffuse oxygen from the multiport ring inlet ( $G$ of Fig. 2). Rectangular openings were cut in the crucible and thermal shield in order to observe the combustion. A Pyrex window covered the opening in the thermal shield.

3. Description of a Typical Experiment

In a typical experiment, the combustor was flushed with oxygen for ten minutes. The sample was then ignited by passing electrical current through an iron fuse wire ( $H$ of Fig. 2) touching the top of the sample. The flow rates were adjusted to preselected initial values for the experiment. Primary and secondary oxygen flows were varied to study the effectiveness of different rates and inlet arrangements. The ratio of diffuse to directed flow was of the order of 3:1 or greater and the total flow rate ranged between 3.5 and 11 moles per minute. At the end of the burn, the combustor was flushed with oxygen and allowed to cool. The ash was collected and weighed. The ash contents of RDF-2 pellets ranged from 15 to $32 \%$ of the initial mass and no unburned organic material was identified in the ash.

In general, the most rapid burning of the sample occurred on the areas 
where the secondary jets of oxygen struck the sample. As the secondary flow velocity was increased, these areas became white hot and the flame became ever more turbulent. Above a critical flow rate, the sample began to fragment vigorously with significant scattering of burning matter and ash. As the temperature of the preheated secondary oxygen was increased, the flow rate at which these changes occurred was lowered. Intense burning occurred in the vertical holes, when present. Bright columns of flame were observed above the holes.

No distinct glow period, analogous to that of the 25 gram samples, was observed. However, CO production tended to be larger during the combustion of the last quarter of the sample. The fraction combusted at any time was assumed to be the ratio of $\mathrm{CO}_{2}$ produced up to that time divided by the total $\mathrm{CO}_{2}$ production. The ratio of total $\mathrm{CO}$ to $\mathrm{CO}_{2}$ ranged from 2 to less than 0.1 mol\%. The time required to burn the last quarter of all RDF-2 samples was always longer than that required to burn the first three quarters of the sample. This was due to the presence of noncombustibles which tended to inhibit the combustion of the last quarter of the unburned material. Peak temperatures of the exhaust gases at the exit stack ranged from 365 to $500^{\circ} \mathrm{C}$, depending upon the experiment, and occurred before half of sample had been combusted. The flame temperature above the sample was determined to be in excess of $1400^{\circ} \mathrm{C}$. The peak temperature of the combustor components nearest the sample ranged from 500 to $1200^{\circ} \mathrm{C}$ for the top of the primary oxygen supply and 440 to $500^{\circ} \mathrm{C}$ for varous parts of the crucible and lower thermal shield. For the most part, only minor surface corrosion of the these components occurred. Actual ignition of the combustor components only took place when the burning sample fell from its support and came into contact with the oxygen inlets. 
4. Summary of Results

The total burn time of RDF-2 pellets was reduced from 77 minutes per kilogram initial mass in the early runs with the experimental arrangement illustrated in Fig. 1 to an acceptable $15 \mathrm{~min} / \mathrm{kg}$ using the arrangement of Fig. 2. As more than one change in sample and/or combustor configuration was made in each trial experiment, interpretation of the effects due to the individual changes tends to be somewhat ambiguous. However, we draw the following conclusions: (1) The introduction of a crucible (having a side wall one half the height of that shown in Fig. 2) caused a reduction in burn times for RDF-2 pellets of $33 \%$. (2) The introduction of vertical holes reduced burn times between 10 and $50 \%$. (3) Burn times for a single tier of lower secondary oxygen inlets (equivalent to $C$ of Fig. 2) were about $50 \%$ less than those obtained using a single tier of upper secondary inlets (which were directed downward at a $45^{\circ}$ angle toward the top edge of the pellet). (4) We found that cellulose pellets were easily ignited and burned smoothly leaving negligible ash. Burn times for cellulose samples were up to $50 \%$ shorter than those for RDF-2. Cellulose appears to be a satisfactory potential calibrant. 


\section{References}

[1] Reilly, M.L., Churney, K.L., Kirklin, D.R., Ledford, A.E. and Domalski, E.S., An oxygen flow calorimeter for kilogram samples of municipal solid waste, Part I. A 25 gram capacity combustion flow calorimeter for determining the calorific value of refuse derived fuels. Accepted for publication in mid-1982 in Resources and Conservation.

[2] Domalski, E.S., Churney, K.L., Reil1y, M.L., Kirk1in, D.R., Ledford, A.E. and Thornton, D.D. 25 gram capacity combustion flow calorimeter, NBSIR 82-2457, March 1982. 
Fig. 1. The 2.5 Kilogram Combustor. A denotes the stack, B the upper thermal shield, $C$ the upper burner jacket, $D$ the annular plate, $E$ the lower burner jacket, $F$ the secondary oxygen inlets, $G$ the support rod, $H$ the sample support and I the primary oxygen inlet.

Fig. 2. The Configuration of the Lower Chamber of the Combustor for Experiments 17 and 18. A denotes the lower thermal shield, B the upper tier of secondary oxygen inlets, $C$ the lower tier. of secondary oxygen inlets, $D$ the sample support, $E$ the primary oxygen inlet, $F$ the lower tier preheat coil, $G$ the multiport ring oxygen inlet, $H$ the iron fuse, I the upper tier preheat coil and $J$ the crucible lid. 


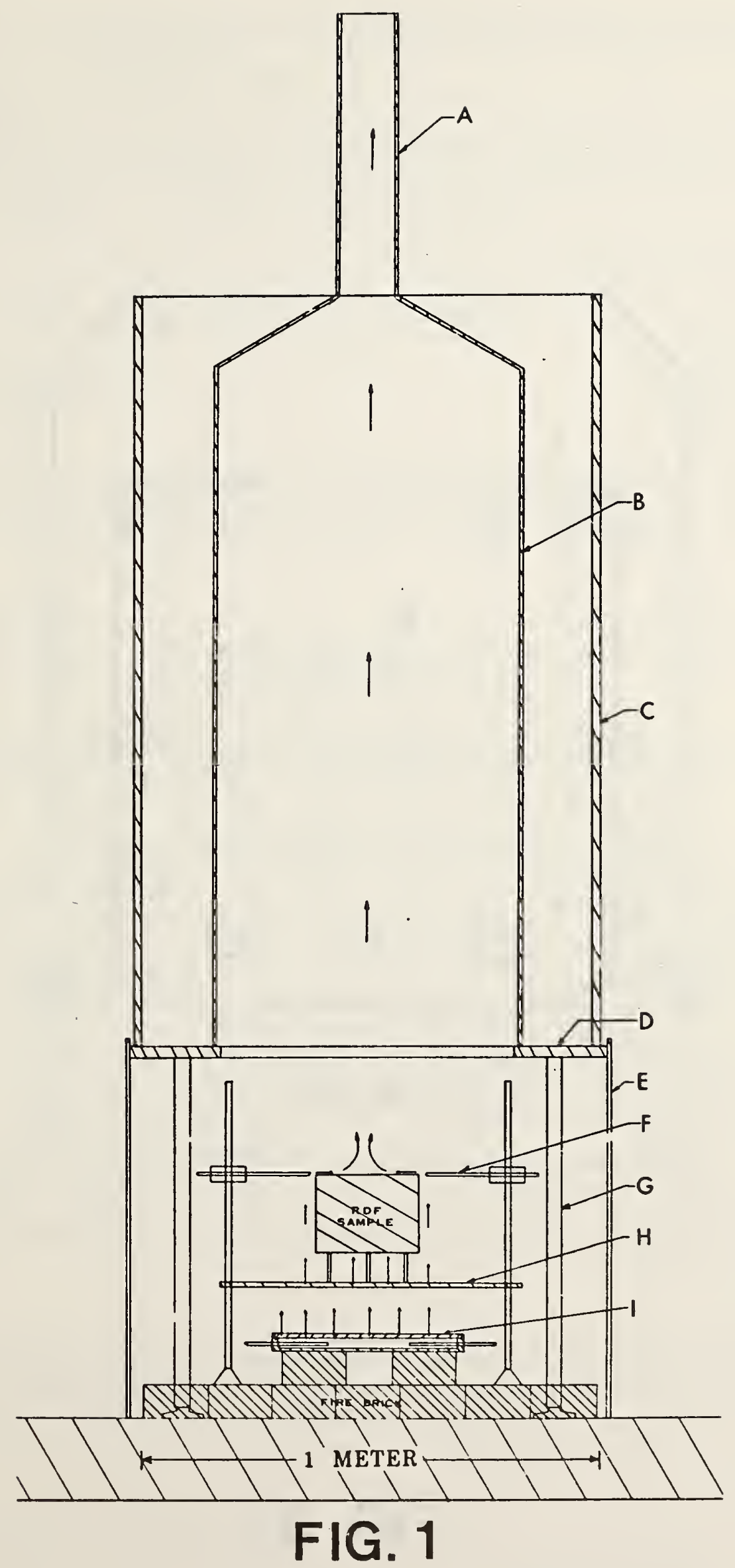




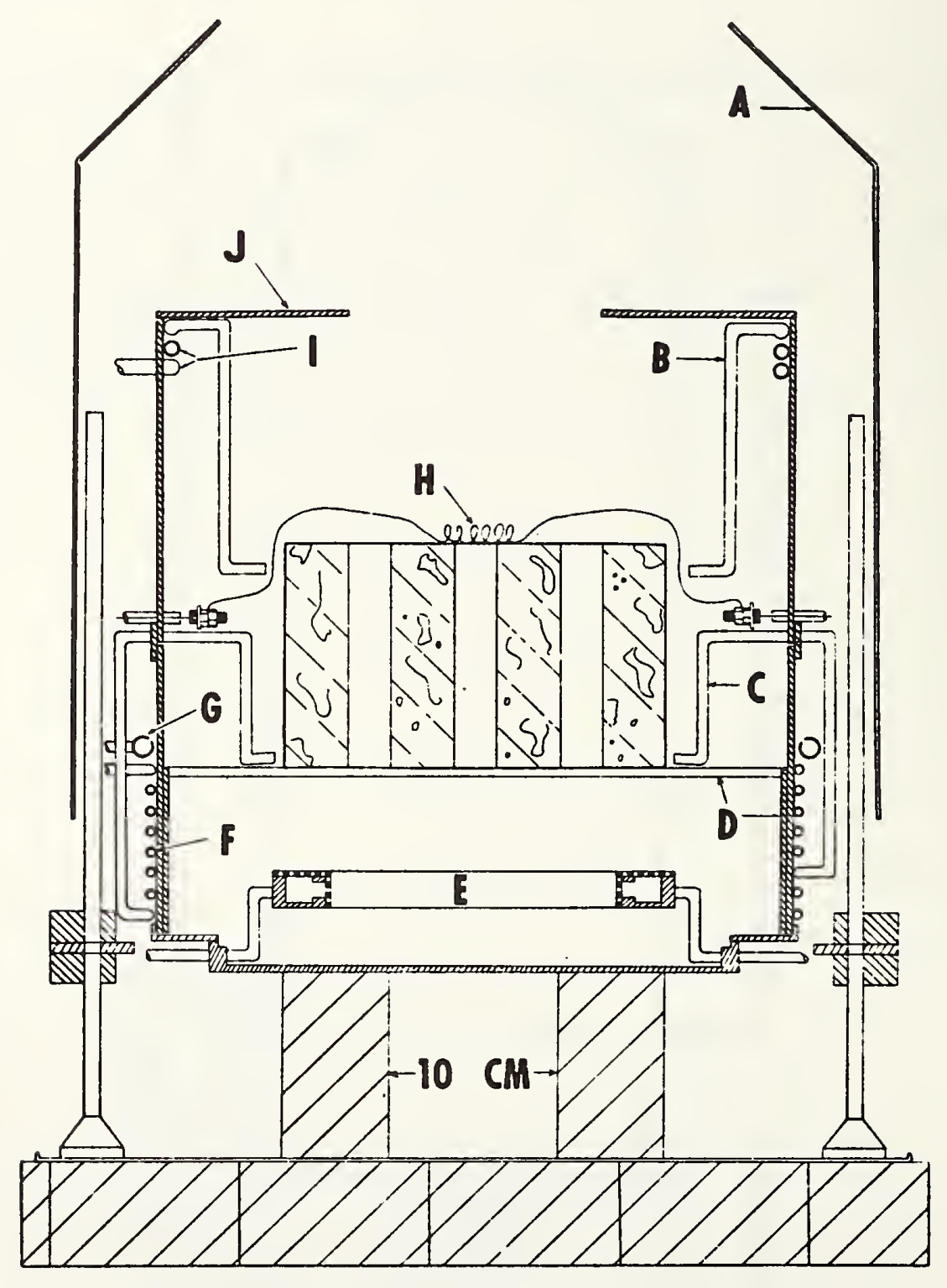

FIG. 2 
NBS.114A (REV. 2-8C)

U.S. DEPT. OF COMM.

BIBLIOGRAPHIC DATA

SHEET (See instructions)
1. PUBLICATION OR
REPORT NO.

NBSIR 82- 2491
2. Performing Organ. Report No. 3. Publication Date

June 1982

4. TITLE AND SUBTITLE

Test Procedures for the Determination of the Gross Calorific Value of Refuse and Refuse-Derived-Fuel of Kilogram-Size Samples Using Constant Pressure Flow Calorimetry. Summary of the 1980 Fiscal Year Results

5. AUThor(S) D.R. Kirklin, J.C. Colbert, K.L. Churney, M.L. Reilly,D.D. Thornton, R.V. Ryan, A.E. Ledford, and E.S. Doma Tski

6. PERFORMING ORGANIZATION (If joint or other than NBS, see instructions)

NATIONAL BUREAU OF STANDARDS

DEPARTMENT OF COMMERCE

WASHINGTON, D.C. 20234

9. SPONSORING ORGANIZATION NAME AND COMPLETE ADDRESS (Street. City, State, ZIP)

7. Contract Grant No.

$E A-77-A-01-6010$

8. Type of Report \& Period Covered

FY 1980 Report

1 Sept 1979-30 Sept 1980

U.S. Department of Energy, Office of Energy From Municipal Waste, Forrestal Bldg.,

7000 Independerice Avenue, S.W., Washington, DC 20585

10. SUPPLEMENTARY NOTES

Funding equally shared by the NBS Office of Recycled Materials and the DoE

Office of Energy from Municipal Waste

$[$ Document describes a computer program; SF-185, FIPS Software Summary, is attached,

11. ABSTRACT (A 200-word or less factual summary of most significant information. If document includes a significant bibliography or literature survey. mention it here)

This report describes the systematic approach used at the National Bureau of Standards to design, construct, and place into operation a constant pressure flow calorimeter which can accommodate kilogram-size samples. The contents of the report are divided into three parts: Part A - The Variability of Municipal Solid Waste and its Relationship to the Determination of the Calorific Value of Refuse-Derived Fuels; Part B - 25 Gram Capacity Combustion Flow Calorimeter; and Part C - Trial Combustions of Kilogram-Size Samples of Municipal Solid Waste.

The primary objective of this effort is to develop a procedure for the determination of accurate calorific values for minimally processed MSW and to correlate these calorific values with those obtained on RDF samples which have been processed to a small particle size.

12. KEY WORDS (Six to twelve entries; alphobetical order: capitalize only proper names; and separate key words by semicolons) Calorific value; flow calorimetry; kilogram-size samples; municipal solid waste; refuse-derived fuel; sample characterization; sample variability

$[-]$ Order From Superintendent of Documents, U.S. Government Printing Office, Washington. D.C. 20402.

14. NO. OF PRINTED PAGES 88

15. Price

[_] Order From National Technical Information Service (NTIS), Springfield, VA. 22161 


